Florida International University

FIU Digital Commons

\title{
The Effect of Proteome and Lipidome on the Behavior of Membrane Bound Systems in Thermally-Assisted Acoustophoresis
}

\author{
Elnaz Mirtaheri \\ Florida International University, emirt001@fiu.edu
}

Follow this and additional works at: https://digitalcommons.fiu.edu/etd

Part of the Biology and Biomimetic Materials Commons, Biomedical Devices and Instrumentation Commons, Biophysics Commons, Other Cell and Developmental Biology Commons, and the Other Materials Science and Engineering Commons

\section{Recommended Citation}

Mirtaheri, Elnaz, "The Effect of Proteome and Lipidome on the Behavior of Membrane Bound Systems in Thermally-Assisted Acoustophoresis" (2019). FIU Electronic Theses and Dissertations. 4056.

https://digitalcommons.fiu.edu/etd/4056

This work is brought to you for free and open access by the University Graduate School at FIU Digital Commons. It has been accepted for inclusion in FIU Electronic Theses and Dissertations by an authorized administrator of FIU Digital Commons. For more information, please contact dcc@fiu.edu. 


\section{FLORIDA INTERNATIONAL UNIVERSITY}

Miami, Florida

THE EFFECT OF PROTEOME AND LIPIDOME ON THE BEHAVIOR OF MEMBRANE BOUND SYSTEMS IN THERMALLY-ASSISTED ACOUSTOPHORESIS

A dissertation submitted in partial fulfillment of the requirements for the degree of DOCTOR OF PHILOSOPHY

in

MATERIALS SCIENCE AND ENGINEERING

by

Elnaz Mirtaheri 


\section{To: Dean John Volakis}

College of Engineering and Computing

This dissertation, written by Elnaz Mirtaheri, and entitled The Effect of Proteome and Lipidome on the Behavior of Membrane Bound Systems in Thermally-Assisted Acoustophoresis, having been approved in respect to style and intellectual content, is referred to you for judgment.

We have read this dissertation and recommend that it be approved.

Shekhar Bhansali

Yiding $\mathrm{CaO}$

Cesar Levy

Ibrahim Tansel

Bilal El-Zahab, Major Professor

Date of Defense: February 22, 2019

The dissertation of Elnaz Mirtaheri is approved.

Dean John Volakis

College of Engineering and Computing

Andrés G. Gil

Vice President for Research and Economic Development and Dean of the University Graduate School

Florida International University, 2019 
(C) Copyright 2019 by Elnaz Mirtaheri All rights reserved. 


\section{DEDICATION}

\section{To my parents,}

without whom none of this would have been possible.

and

To my brother, Ali,

who always encouraged me to go on every adventure, especially this one! 


\section{ACKNOWLEDGMENTS}

I would like to express my gratitude to all the people who have helped me and given me support throughout the completion of my PhD studies. First, to my parents, Mehran and Mohammad, who have always loved and supported me. Of course, I could not have done this without the support and encouragement of other family members including my brother Ali.

I would like to thank my advisor, Dr. Bilal El-Zahab, for guiding me and helping me reach my potential. His technical guidance, support, and encouragement were very important factors in my completing this effort. This dissertation would not have been possible without the support of my committee members Dr. Shekhar Bhansali, Dr. Cesar Levy, Dr. Ibrahim Tansel and Dr. Yiding Cao all of whom deserve special thanks for providing support, professionally and personally, along the way.

Shervin, Maedeh, Azadeh, Amirali and Arezou I considered whom my best friends at FIU, deserve a special mention. I am indebted to you both for your support and friendship over these years. A very special acknowledgement goes to Ata, who encouraged me and given me unwavering support from the very first days of my Ph.D. studies and even before, and constantly made me feel like I am not alone. Finally, I would like to acknowledge the teachers, professors, and other professionals who either directly trained me, or inspired me in some way. 


\title{
ABSTRACT OF THE DISSERTATION \\ THE EFFECT OF PROTEOME AND LIPIDOME ON THE BEHAVIOR OF MEMEBRANE BOUND SYSTEMS IN THERMALLY-ASSISTED ACOUSTOPHORESIS
}

\author{
by \\ Elnaz Mirtaheri \\ Florida International University, 2019 \\ Miami, Florida \\ Professor Bilal El-Zahab, Major Professor
}

Changes in the biomechanical properties of cells accompanying the development of various pathological conditions have been increasingly reported as biomarkers for various diseases, including cancers. In cancer cells, the membrane properties have been altered compared to their healthy counterparts primarily due to proteomic and lipidomic dysregulations conferred by the underlying pathology. The separation and selective recovery of these cells or extracellular vesicles secreted from such cells is of high diagnostic and prognostic value.

In this dissertation, the research builds on thermally-assisted acoustophoresis technique which was developed in our laboratory for the separation of vesicles of the same size, charge and shape yet with varying physical properties. This technique uses the inherent thermotropic behavior of lipid membrane to identify a distinct acoustic contrast temperature $\left(\mathrm{T}_{\phi}\right)$ for each individual composition under acoustophoresis. By tuning the temperature, the acoustic contrast factor $(\Phi)$ of vesicle systems experience a signature temperature at which sign of $\Phi$ switches from positive to negative. This temperature is defined as the acoustic contrast temperature, $T_{\phi}$. Since various vesicles systems have distinct $\mathrm{T}_{\phi}$ values, it allowed the development of a separation method of vesicles based on their membrane properties, with target outlet purities exceeding $95 \%$. 
Over-expression and under-expression of proteins play crucial roles in the functionality of cells and can be indicators of pathological disorders. Using systematic designed experiments, the effect of membrane protein content in vesicles was studies. Using three different transmembrane peptides (gramicidin, alamethicin and melittin), the thermo-acoustofluidic properties of vesicles were studied in an in-house built lab-on-chip to assess the separation efficiencies for various protein contents.

To demonstrate the utility of this method and its performance on real biological samples, the effect of proteins on thermally-dependent acoustic properties of red blood cells were investigated. The separation of red blood cells based on expressed membrane proteins of different contents proved to yield to distinctive $\mathrm{T}_{\phi}$ values that afforded the separation of the cells. The simplicity, rapidity, and label-free nature of this approach holds promise as a diagnostic and separation tool for cells affected by diseases that affect the physical properties of membrane and extracellular vesicles such as exosomes and microvesicles. 


\section{TABLE OF CONTENTS}

CHAPTER

PAGE

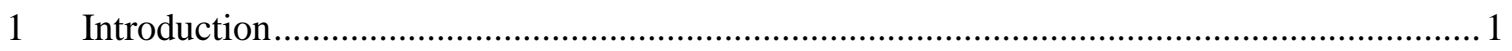

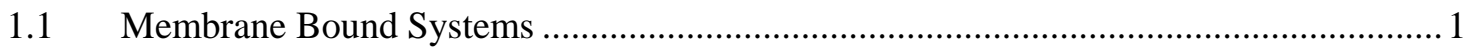

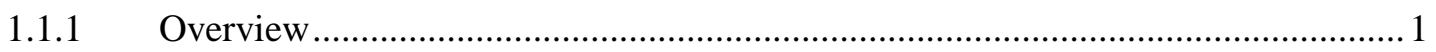

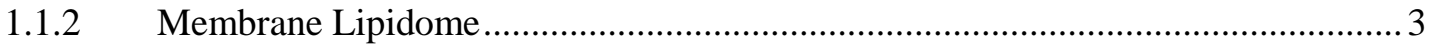

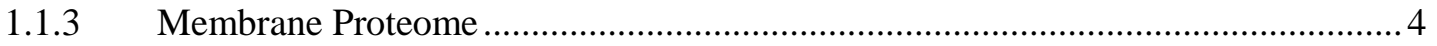

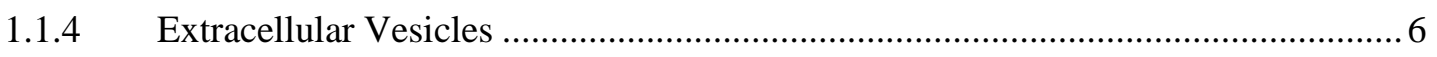

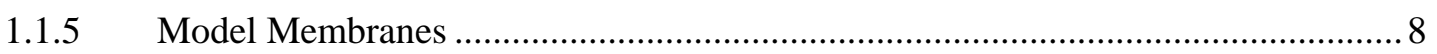

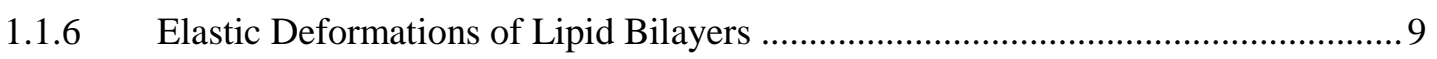

1.2 Separation and Sorting of Particles Using Microfluidics............................................ 13

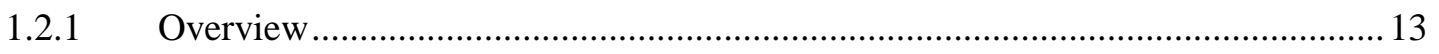

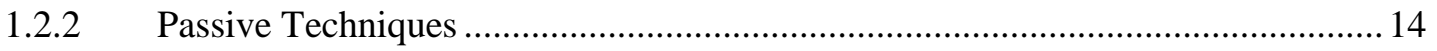

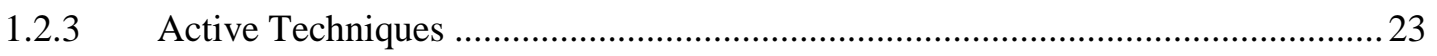

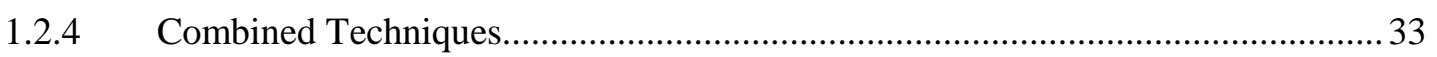

$1.3 \quad$ Acoustophore

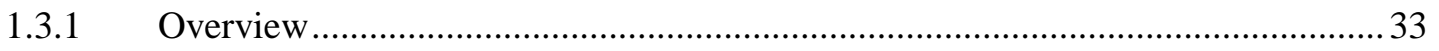

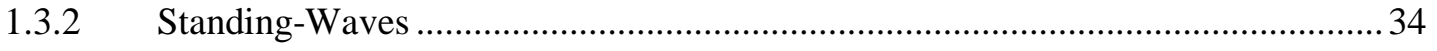

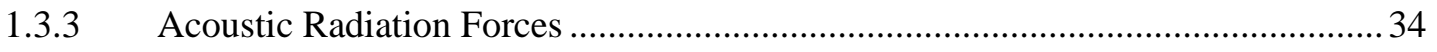

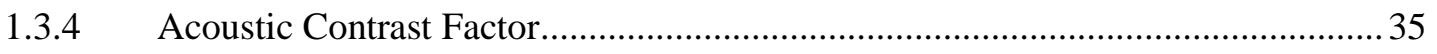

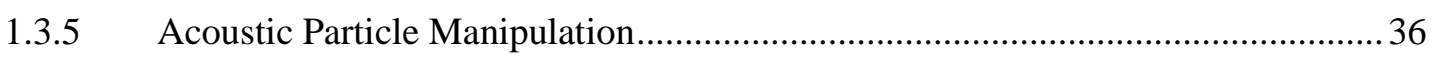

1.3.6 Acoustophoresis of Biological Particles ........................................................... 41

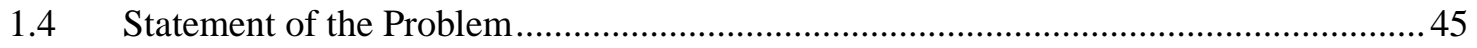

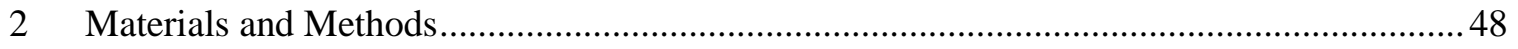

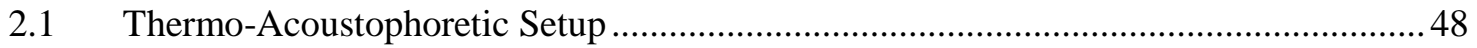

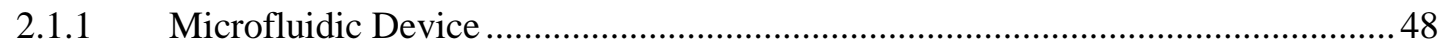

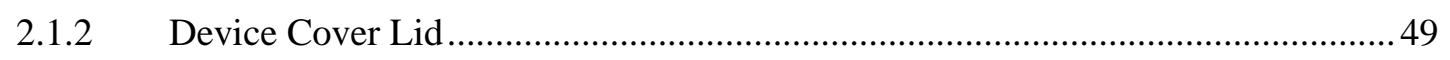

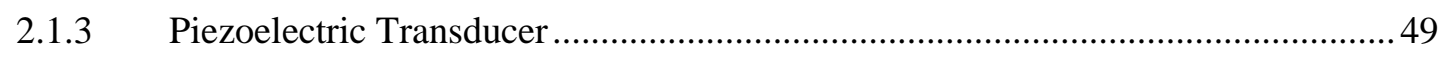

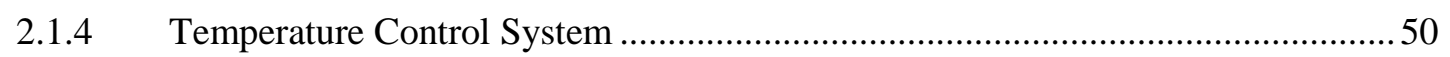

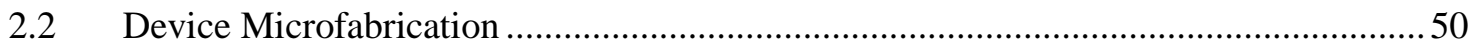

2.2.1 Mask Design and Pattern Generation..................................................................5

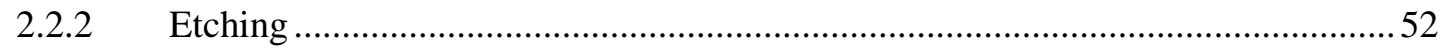

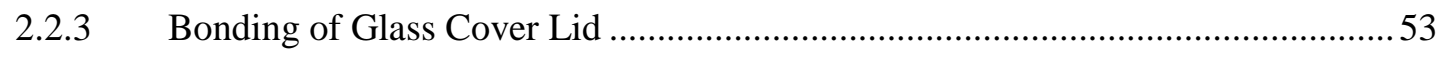

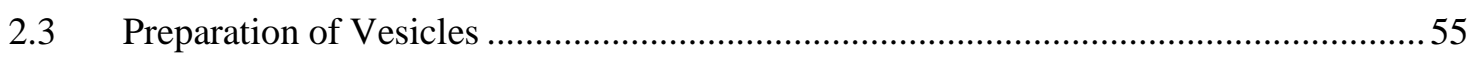

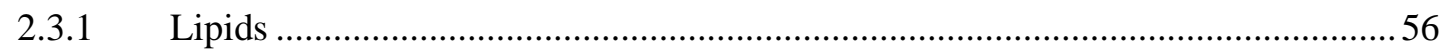




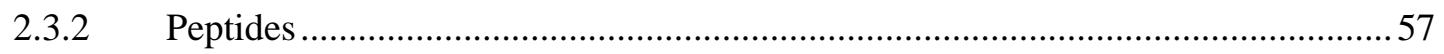

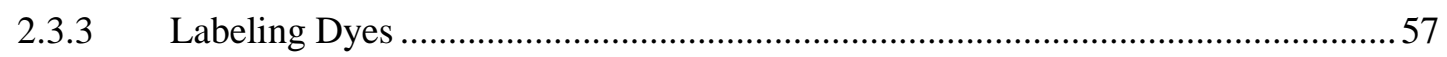

3 The Effect of Proteins on Thermo-Acoustofluidic properties of Vesicles.............................59

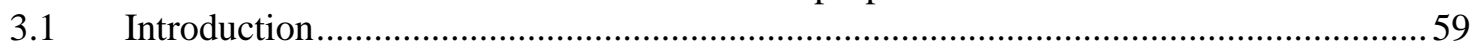

3.2 Transmembrane Peptides: Gramicidin, Alamethicin and Melittin ...............................63

3.3 Thermo-Acoustofluidic Behavior Vesicles Containing Model Proteins.........................65

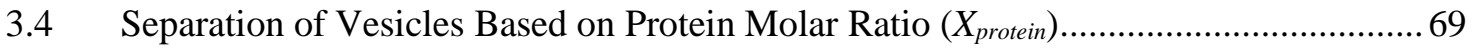

3.5 Analysis of Separation Performance Under Different Conditions .................................. 73

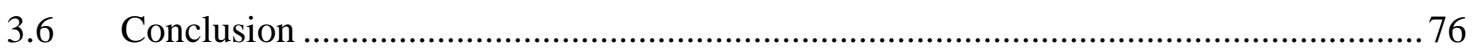

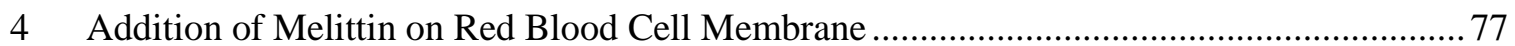

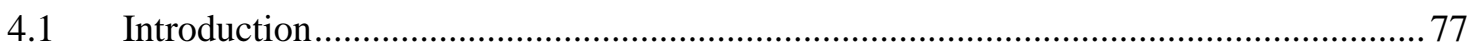

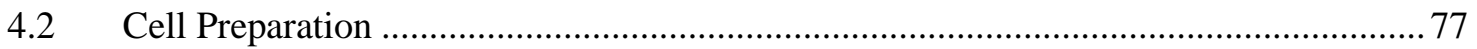

4.3 The Effect of Melittin Concentration on Red Blood Cell Membrane ............................. 77

4.4 Thermo-Acoustofluidic Behavior RBCs Containing Melittin ..................................... 81

5 Separation of Vesicles from Cancer Cells Using Thermally-assisted Acoustophoresis ........ 84

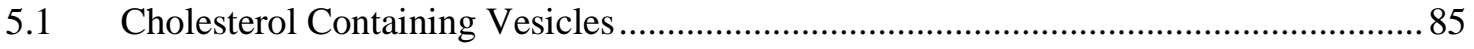

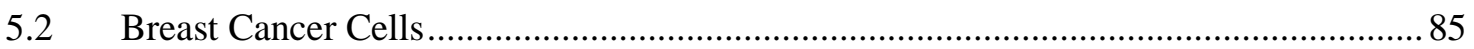

5.3 Thermo-Acoustophoretic Behavior of Vesicles in a Media Containing Cancer Cells .. 86

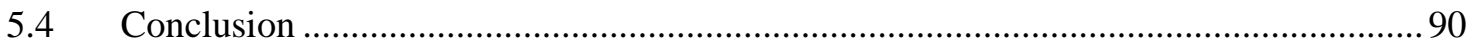

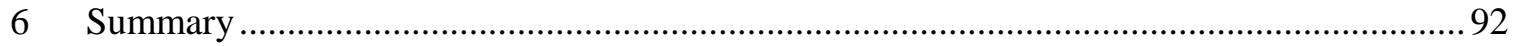

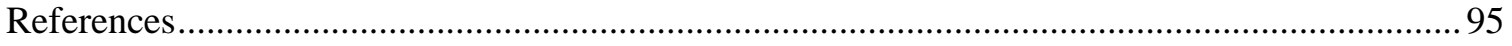

VITA 


\section{LIST OF TABLES}

TABLE

PAGE

Table 2-1 Characterization of APC 850 and 851 piezoelectric transducers

50 


\section{LIST OF FIGURES}

Figure 1-1 a) Davson-Danielli model (1935) showing a lipid bilayer including proteins in the form of a distinct layer. b) The "fluid mosaic model" of the membrane proposed by Singer and Nicolson (1972) showing a lipid bilayer and its heterogeneously distributed proteins (Reprinted with permission from ${ }^{6}$ ).

Figure 1-2 Schematic illustration of a biological membrane, showing a), the saturated and unsaturated lipid molecules, cholesterol, the actin network, glycolipids, glycosylphosphatidylinositol (GPI)-anchored proteins, lipidated proteins, and transmembrane proteins. The subdomains enriched in saturated lipids, cholesterol, (GPI)-anchored and lipidated proteins are known as lipid rafts, characterized by their higher packing order and decreased fluidity compared to the non-raft domains. b) Uneven component distribution in each leaflet and among the inner and outer leaflets of a biological membrane leads to a membrane organization consisting of various small raft-like and non-raft domains. (Reprinted with permission from ${ }^{18}$ ).

Figure 1-3 Formation of MVs and exosomes. MVs are shed directly from the membrane whereas exosomes are first formed by budding into early endosomes or multivesicular endosomes (MVEs) and then released in the extracellular environment through fusion of MVEs with plasma membrane (Reprinted with permission from ${ }^{19}$ ).

Figure 1-4 Schematic overview of modes of elastic deformation in bilayer membranes: Dilation or stretching, bending, shearing and monolayer slipping (Reprinted with permission from ${ }^{46}$ )... 11

Figure 1-5 shows the dependence of kckT on DHH for seven PC lipids. It also shows the effect of temperature on the bending rigidity for DOPC (Reprinted with permission from ${ }^{49}$ ).

Figure 1-6 Schematic presentation of pinched flow fractionation (PFF) technique (Reprinted with permission from ${ }^{69}$ )

Figure 1-7 Schematic representation of a flow fractionation device (Reprinted with permission from ${ }^{74}$ ).

Figure 1-8 Schematic of particle manipulation in microvortex manipulation (MVM) technique (Reprinted with permission from ${ }^{75}$ ).

Figure 1-9 DLD sorting of particles through zigzag mode and displacement mode (Reprinted with permission from ${ }^{78}$ ).

Figure 1-10 (a) Schematic illustration of Zweifach-Fung effect for the tendency of particles at a bifurcation. (b) Three different scenarios of particles migration at the bifurcation (Reprinted with permission from ${ }^{84}$ )

Figure 1-11 Schematic of microfilter designs, a) Weir type filter which enables the separation of particles based on size, b) Pillar type filter which contains pillar with designed spacing in 
order to hinder the passage of large particles, c) Cross-flow filters in which the flow direction is perpendicular to the array of pillars (Reprinted with permission from ${ }^{65}$ ).....

Figure 1-12 Schematic of microfilter design with multi membrane structure each of which has a different pore size (Reprinted with permission from ${ }^{87}$ ).

Figure 1-13 Fundamental of hydrodynamic filtration showing a separation capability depending on volume flow rate: (a) side channel has a small volume flow rate therefore particles will not pass through the side channel, (b) the volume flow rate is medium, therefore the side channel only allows the smaller size of the particles and particles with the size larger than a critical value are not able to pass into the side channel, (c) the side channel has the high volume flow rate, therefore the minimum size of the particles that can flow through the channel increased. (d) Schematic design of a microchannel for sorting and separation of multiple particles (Reprinted with permission from ${ }^{92}$ ).

Figure 1-14 Schematic illustration of (a) a micro-hydrocyclone, and (b) forces acting on a particle in spiral flow inside the cylinder (Reprinted with permission from ${ }^{94}$ ).

Figure 1-15 Schematic illustration of a nonuniform electric field with positive and negative DEP (Reprinted with permission from ${ }^{95}$ ).

Figure 1-16 Showing the different modes of field flow fractionation (Reprinted with permission from ${ }^{66}$ ).

Figure 1-17 Schematic presentation of the application of a multitarget magnetic-activated cell sorter (MT-MACS) (Reprinted with permission from ${ }^{100}$ ).

Figure 1-18 Schematic illustration of free-flow magnetophoresis for the separation of particles based on their size and magnetic properties (Reprinted with permission from ${ }^{103}$ )......

Figure 1-19 Schematic separation of particles using split-flow lateral-transport thin (SPLITT) (Reprinted with permission from ${ }^{66}$ ).

Figure 1-20 (a) optical lattice principles, and (b) Separation of particles using an optical lattice (Reprinted with permission from ${ }^{109}$ ).

Figure 1-21 Optical micrograph of an LOT microfluidic device (Reprinted with permission from $111)$.

Figure 1-22 Representing a) an acoustically actuated microfluidic device. The transversal direction of the channel and the flow direction in the channel are shown as $y$ and $x$, respectively. b) schematic of ultrasonic standing wave along with its pressure nodes and antinodes and the direction of migration for different particles in acoustic standing wave (Reprinted with permission from ${ }^{129}$ ).

Figure 1-23 Illustration of particle movement in a standing wave acoustophoresis: (a) In the absence of acoustic radiation. (b) When acoustic radiation is activated at a half-wavelength resonance, resulting in the migration of particles toward the central pressure node which is shown with a purple line. The blue arrows show the direction of net force applied on the particles 
in such acoustic radiations. (c) the accumulation of the particles at the central node due to the acoustic radiation force. (Reproduced with permission from ${ }^{124}$ ).

Figure 1-24 Showing the acoustic sedimentation technique: (a) Prior to the acoustic actuation particles are randomly dispersed in the channel. (b) Upon actuation particles start to accumulate at the center line of the channel which is the pressure node at half-wavelength resonance mode. (c) Accumulated particles start to sediment due to gravity. (Reproduced with permission from $\left.{ }^{124}\right)$.

Figure 1-25 Schematic of a continuous microfluidic channel for the separation of particles from their carrier fluid. Particles are focused in the middle of the channel and are separated from the middle outlet and the medium is collected from the upper and lower outlets (Reproduced with permission from ${ }^{132}$ ).

Figure 1-26 Washing particles from one medium to another using acoustic radiation under laminar flow. When the acoustic radiation is adjusted to produce the pressure node in the second medium, particles tend to migrate from the first carrier medium to the second one (Reproduced with permission from ${ }^{133}$ ).

Figure 1-27 Acoustic radiation resulted in an improvement in capturing bacteria by the biosensor. The sanding wave in this case is working in a quarter-wavelength resonance mode so that the pressure node is formed on the biosensor side wall, therefore increase the capture of bacteria on the sensor (Reproduced with permission from ${ }^{135}$ )

Figure 1-28 Acoustofluidic separation of particle based on their size using a quarter-wavelength resonance. The size of the particle determines the magnitude of the force exerted on them. Based on the difference in size, particles are focused in different locations at the standing wave (Reproduced with permission from ${ }^{124}$ ).

Figure 1-29 Removing proteins from red blood cells (RBC) using acoustophoresis. Protein-free $\mathrm{RBCs}$ are collected from the center outlet (picture on the right shows the outlet and collected $\mathrm{RBCs}$ ). (Reprinted with permission from ${ }^{143}$ ).

Figure 1-30 a) Showing the schematic of taSSAW device. b) The node and antinode in taSSAW are at an angle with regards to the flow direction. As a result, particles with larger sizes such as CTCs undergo greater acoustic force in comparison with WBCs, they therefore migrate to farther distances perpendicular to the flow direction. c) Image of an actual taSSAW microfluidic system. d) Showing a mixture of HeLa cells and WBCs inserted in the channel with no acoustic actuation. All the particles are collected from the lower outlet and no separation occurs. e) The same mixture upon acoustic actuation are separated based on their size. HeLa cells are larger, so they experience greater force and migrate toward the upper outlet, while WBCs are collected from the lower outlet. (Reprinted with permission from ${ }^{146}$ ).

Figure 1-31 Showing the binding of elastomeric particles to cells for the separation of cells: a) Bright-field, and b) Fluorescent micrograph of SA-adsorbed particles that are attached to KG1a cell (labeled with green dye). c) Showing the migration of cells with their attached elastomeric particles as the acoustic actuation turns on. d) From left to right: intensity distribution of SA-adsorbed particles, biotinylated polystyrene microbeads and complexes of particles along the channel with the width of W. e) The intensity profiles for the separation of particle-bounded cells from particle-free cells. f) fluorescence micrograph of KG-1a cells (green 
labeled) focused at node and elastomeric particles tagged with Nile red dye focused at the antinode. And g) the separation of SA-adsorbed elastomeric particles attached to the anti-CD34biotin cells (green labeled) from the cells without anti-CD34-biotin (red labeled). (Reprinted with permission from ${ }^{148}$ ).

Figure 1-32 Schematic representation of a separation event (left), and building blocks of a lipid vesicle containing a transmembrane protein.

Figure 2-1 Schematic illustration of a microfluidic channel resonating in transversal direction with optimum dimensions. (Reprinted with permission from ${ }^{149}$ )

Figure 2-2 Image of the microfluidic separation device designed and fabricated for this study using standard photolithography procedure.

Figure 2-3 Process flow of the fabrication of microfluidic device showing the photolithography process of the front side of silicon wafer after cleaning with acetone, IPA and HMDS. The process flow consisted of photoresist spin coating, followed by a soft bake, exposure and development in the proper developer solution. The developed pattern was then etched with RIE to remove nitride layer in the patterned area and produce a hard mask. Then, the exposed silicone was etched through with a wet chemistry etching process in $\mathrm{KOH}$. After etching the microfluidic channel, the remaining nitride layer was removed using an aluminum etchant and followed by cleaning, drying and anodic bonding of glass cover.

Figure 2-4 Optical micrograph of the DMPC vesicles prepared by the described method.....

Figure 3-1 Schematic of the acoustofluidic device in a separation experiment; two types of EVs injected through the same inlet initially flow randomly but upon actuation of the device at a specific frequency and temperature focus in distinct streamlines running along different outlets. The number and position of streamlines is determined by the frequency or wavelength $(\lambda)$ of the actuation signal which is in turn adjusted according to the width of the channel in the separation region. Inset on the left shows an optical micrograph of the vesicles with a scale bar of $10 \mu \mathrm{m}$. The image on the right is the actual microfluidic chip.

Figure 3-2 (a) The building blocks of the lipid vesicles used in this study: amphiphilic lipids (e.g. DPPC), and transmembrane peptides (e.g. Gramicidin D); (b) Upon hydration under proper conditions, the lipids arrange in bilayers to self-assemble to form liposomes of average $1 \mu \mathrm{m}$ incorporating transmembrane peptides which, depending on their type and concentration, can assume various conformations (e.g. transmembrane pores) in the membrane.

Figure 3-3 The acoustic contrast temperature, $T_{\Phi}$, vs. composition for (a) DMPC and (b) DPPC vesicles, upon the introduction of gramicidin. The error bars represent the maximum and minimum values recorded for each system in at least five independent experiments using separate preparations

Figure 3-4 The acoustic contrast temperature, $T_{\Phi}, v s$. composition for (a) DMPC and (b) DPPC vesicles, upon the introduction of alamethicin. The error bars represent the maximum and minimum values recorded for each system in at least five independent experiments using separate preparations 
Figure 3-5 The acoustic contrast temperature, $T_{\Phi}$, vs. composition for (a) DMPC and (b) DPPC vesicles, upon the introduction of melittin. The error bars represent the maximum and minimum values recorded for each system in at least five independent experiments using separate preparations. The blue horizontal line indicates the $T_{\Phi}$ of pure DMPC and DPPC systems.

Figure 3-6 Recording of temperature vs. time under different heating rates $\left(5,10\right.$ and $15^{\circ} \mathrm{C} \cdot \min ^{-}$ ${ }^{1}$ ) when measuring $T_{\Phi}$ of DPPC vesicle systems with $\mathrm{Xg}=1 \%$. A $1^{\circ}$ difference can be detected between the point at which the stagnation phase is observed and the point at which the antinodal migration is initiated $\left(T_{\Phi}\right)$. The plateau, evident in $5{ }^{\circ} \mathrm{C} \mathrm{min}-1$ heating rate, is not as evident in $10^{\circ} \mathrm{C} \mathrm{m^{-1 }}$ and is completely absent in faster heating rates $\left(15^{\circ} \mathrm{C} \mathrm{min}^{-1}\right)$.

Figure 3-7 Fluorescence micrographs of the experiment involving the separation of DPPC vesicles with $X_{g}=7.5$ (green) and $X_{g}=10 \%$ (blue). (a) Vesicles are randomly dispersed in the microchannel at $37^{\circ} \mathrm{C}$ with no acoustic actuation. (b) The same vesicles in figure (a) 3 seconds after setting the acoustic transducer to the first harmonic frequency undergo a nodal focusing at the same temperature. (c) After sweeping up the temperature to $\sim 39{ }^{\circ} \mathrm{C}, X_{g}=7.5$ vesicles (green) begin to migrate toward the antinodes while $X_{g}=10 \%$ vesicles (blue) remain at the pressure node of the channel (scale bar in all images is $100 \mu \mathrm{m}$ ). Vesicles now flowing along distinct streamlines can be collected from separate outlets.

Figure 3-8 Relative values of $\Phi$ for DPPC vesicles $X_{g}=7.5$ (green) and $X_{g}=10 \%$ (blue). Inset: The allowable separation window for a mixed system composed of these two vesicle types is marked in blue, outlining the temperature range within which separation could be achieved due to opposite migrations of the vesicles. The relative values of $\Phi$ were calculated using vesicle compressibility data from the literature.

Figure 3-9 (a) and (b) 3D mapping of a separation event constructed using the fluorescent intensities of the blue and green channels across an area of approximately 400 by $600 \mu \mathrm{m}$ in the channel at respectively $37{ }^{\circ} \mathrm{C}$, where the acoustic transducer is on but $T<T_{\Phi, 7.5 \%}<T_{\Phi, 10 \%}$, leading to the focusing of both systems in the center node, and at $39{ }^{\circ} \mathrm{C}$, where the acoustic transducer is on and $T_{\Phi, 7.5 \%}<T<T_{\Phi, 10 \%}$, causing the vesicles with $7.5 \mathrm{~mol} \%$ gramicidin to migrate towards the antinodes. (c) Outlet purity as a function of vesicles concentration in the channel (parts of the original suspension: parts of the diluent). Original concentration of the vesicles, 1:0, approximately corresponded to $10^{6}$ vesicles per $\mathrm{ml}$ of the suspension. (d) Outlet purity as a function of $\Delta T_{\Phi}$, i.e. the width of the allowable separation window, corresponding to the difference between the $T_{\Phi} \mathrm{S}$ of constituent vesicle types of the binary mixture. (In all separation experiments, the system of lower $T_{\Phi}$ was labelled green and the one of higher $T_{\Phi}$ was blue. In addition, the flow rate in all experiments was $100 \mu \mathrm{hr}{ }^{-1}$, with $f_{1}$ and $\mathrm{V}_{\mathrm{pp}}$ set to $1.4 \mathrm{MHz}$ and $50 \mathrm{~V}$, respectively.) The separation temperature was always selected at the middle temperature between $\mathrm{T}_{\Phi 1}$ and $\mathrm{T}_{\Phi 2}$.

Figure 4-1 Optical microscope image of RBCs containing $1 \mu \mathrm{M}$ Melittin at (a) 25 and (b) $37^{\circ} \mathrm{C}$.

Figure 4-2 Optical microscope image of RBCs containing $5 \mu \mathrm{M}$ Melittin at (a) 25 and (b) $37^{\circ} \mathrm{C}$.

Figure 4-3 Optical microscope image of RBCs containing $10 \mu \mathrm{M}$ Melittin at (a) 25 and (b) $37^{\circ} \mathrm{C}$. 
Figure 4-4 Optical microscope image of RBCs containing $20 \mu \mathrm{M}$ Melittin at (a) 25 and (b) $37^{\circ} \mathrm{C}$.

Figure 4-5 Optical micrographs of red blood cells response to acoustic actuation at the first harmonic frequency at $37^{\circ} \mathrm{C}$; (a) when no melittin is present in the membrane, a nodal focusing occurs which is stable upon increasing temperature up to $42{ }^{\circ} \mathrm{C}$, and (b) when $20 \mu \mathrm{M}$ melittin was added to the system; an antinodal focusing is observed even at room temperature, implying a decrease in $\Phi$ to negative values.

Figure 5-1 a) Focusing of a system consisting of MCF-7 cells (green) and 80:20 DMPC:CHOL vesicles (red) at $20{ }^{\circ} \mathrm{C}$ when the acoustic transducer is on. b) The same system after the temperature of the channel has reached $31^{\circ} \mathrm{C}$

Figure 5-2 a) Thermo-acoustophoretic behavior of a system consisting of MCF-7 cells (green), 90:10 DMPC:CHOL (blue) and 80:20 DMPC:CHOL vesicles (red) at $27{ }^{\circ} \mathrm{C}$ when the acoustic transducer is on. b) The same system at the second stage of the channel where the temperature of is kept at $31^{\circ} \mathrm{C}$.

Figure 5-3 Purity of the vesicles and cells collected from the single-stage and two-stage devices. The first two series of clustered columns on the left present the data obtained from experiments in the single-stage device with 90:10 DMPC:CHOL/MCF-7 and 80:20 DMPC:CHOL/MCF-7 systems. The right-hand side columns show the purity data obtained from the separation study performed in the two-stage device with the three-component system. $\mathrm{T}_{1}$ indicates the temperature of the first Peltier element $\left(1^{\text {st }}\right.$ stage of the channel $)$ and $T_{2}$ indicates the temperature of the second Peltier element ( $2^{\text {nd }}$ stage). Since the single-stage device was equipped with only one Peltier element, only one temperature is reported for the experiments performed in that device. 


\section{ABBREVIATIONS AND ACRONYMS}

PEs

Phosphatidylethanolamines

PCs

Phosphatidylcholines

PSs

Phosphatidylserines

PGs

Phosphatidylglycerols

PIs

Phosphatidylinositols

GPI

Glycosylphosphatidylinositol

EVs

Extracellular Vesicles

MVs

Microvesicles

MVEs

Multi-Vesicular Endosomes

SLB

Supported Lipid Bilayer

SUVs

Small Unilamellar Vesicles

LUVs

Large Unilamellar Vesicles

GUVs

Giant Unilamellar Vesicles

LOC

Lab-on-Chip

PFF

Pinched flow fractionation

MVM

Microvortex manipulation

DLD

Deterministic lateral displacement

CTCs

Circulating tumor cells 
DEP

$\mathrm{CM}$

DACS

FFF

FACS

HGMF

MACS

MT-MACS

MFS

MBs

MPA

SPLITT

HOTs

LCD

AOD

LOT

ODEP

OC

EOF
Dielectrophoresis

Clausius-Mossotti factor

DEP-activated cell sorting

Field flow fractionation

Fluorescence-activated cell sorting

High-gradient magnetic field

Magnetic cell separation system

Multi-target magnetic-activated cell sorter

Ferromagnetic strips

Magnetic beads

Micropillar array

Split-flow lateral-transport thin

Holographic optical tweezers

Liquid crystal display

Acousto-optic deflector

Asymmetric scanning line optical tweezer

Optically induced electrophoresis

Optical chromatography

Electro-osmotic flow 
Gram

Alm

Mel
Gramicidin

Alamethicin

Melittin 


\section{Introduction}

\subsection{Membrane Bound Systems}

\subsubsection{Overview}

Cells and some of their compartmental organelles are confined by biological membranes to maintain their integrity and establish a boundary between their respective inside and outside environments. In addition to their function as selective barrier for some biomolecules, membranes act as communication media with the outside environments (extracellular milieu in the case of plasma membrane). They, therefore, play a crucial role in cellular functions such as signaling, transportation, recognition, immunological, and enzymatic activities. The term plasma membrane was first used by Pfeffer in 1877 when he, while studying the osmotic pressure within cells, noticed a thin layer surrounding the protoplasm of cells. ${ }^{1}$ In 1895, Charles Ernest Overton hypothesized that the plasma membrane has the properties of oil and he named this layer as "lipoids" which is made of lipids and cholesterols. ${ }^{2}$ Langmuir in 1917 developed a monolayer of lipids with the polar head group of its molecules facing toward the water whereas the hydrophobic hydrocarbons were pointing toward the air phase. ${ }^{3}$ Following this interface model, Gorter and Grendel in 1925 found that cell membranes are composed of two opposing lipid layers (a bilayer) in which the polar head groups of lipid molecules face the aqueous environment. ${ }^{4}$ In 1935, Danielli and Davson completed the membrane model by taking into account the existence of considerable amount of proteins in the membrane (Fig. 1a). ${ }^{5}$ Based on the results obtained from X-ray crystallography and electron micrographs of proteins in 1960s, a more comprehensive understanding of proteins in the membrane was achieved. In 1972, Singer and Nicolson introduced a model for biological membranes which is known as the "fluid mosaic model", based on which the plasma membrane is composed of lipids and proteins ${ }^{6}$ (Fig. 1b). The membrane proteins can be divided into two main categories: proteins that are present on the surface of the membrane and are called "peripheral proteins" and the ones which are either adsorbed to the membrane surface or span through the 
membrane and are called "integral proteins". Peripheral proteins, in contrast to the integral proteins can be easily detached from the membrane. This model considers a homogeneous distribution of proteins and lipids in the membrane while implying that the lipid membrane is asymmetric meaning that the composition or other features are notably different on the inside and outside of the membrane. ${ }^{7}$ In 1984 another model for membranes was proposed by Mouritsen and Bloom. ${ }^{8}$ This model, called the "mattress model", is based on the interactions due to the differences in the hydrophobic length of the protein and lipid molecules in the membrane. To be more specific, the thickness of the membrane bilayer is approximately $5 \mathrm{~nm} .{ }^{9}$ In cases where the membrane proteins have a thicker or thinner hydrophobic core, the lipid membrane must compensate for the changes in thickness to avoid unfavorable hydrophobic interactions. This phenomenon is called "hydrophobic matching". In the mattress model the interactions of the proteins with their surrounding lipids due to hydrophobic matching may result in the accumulation of certain lipids around the protein as well as aggregation and clustering of proteins..$^{9,10}$

The composition, organization and structure of lipid membranes are exceedingly complex. A plasma membrane is usually composed of approximately $50 \mathrm{wt} . \%$ lipids, $40 \mathrm{wt} . \%$ proteins and 10

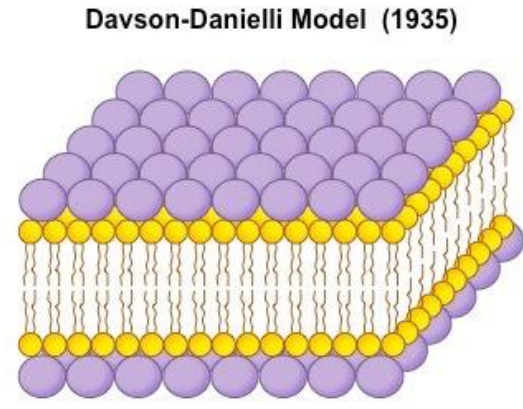

Proteins form distinct layers (sandwich)
Singer-Nicolson Model (1972)

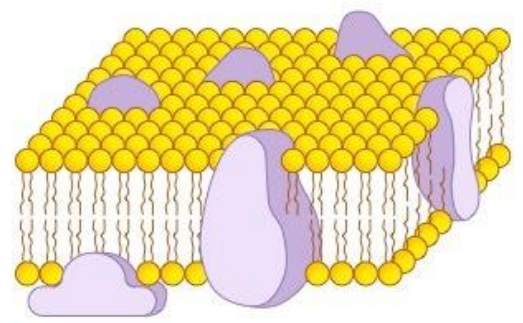

Proteins embedded within bilayer (fluid-mosaic)

Figure 1-1 a) Davson-Danielli model (1935) showing a lipid bilayer including proteins in the form of a distinct layer. b) The "fluid mosaic model" of the membrane proposed by Singer and Nicolson (1972) showing a lipid bilayer and its heterogeneously distributed proteins (Reprinted with permission from $\left.{ }^{6}\right)$. 
wt.\% carbohydrates. Structurally, a plasma membrane consists of three distinguishable layers: 1) a lipid bilayer which lies in the middle and enclosed in various biomolecules, 2) a glycoproteinpolysaccharide covering the extracellular side of the membrane (in the case of bacterial cell structure, peptidoglycan, a polysaccharide backbone, is located right outside of the cytoplasmic membrane instead of glycoprotein, and 3) a cytoskeleton: a network of interlinking filaments and tubules in the intracellular environment. ${ }^{11}$ The main function of these protein filaments is to provide a mechanical resistance to deformation, and therefore, maintaining cell's shape. They are also responsible for cell migration and intracellular transport such as the movement of vesicles and organelles within the cells. ${ }^{12}$ Also notable, is an additional protective layer covering outside of the cells which enhances cell-cell recognition, communication and intercellular adhesion. This layer is called glycocalyx and is made of carbohydrates attached to the tails of membrane glycolipids and glycoproteins. ${ }^{11}$

\subsubsection{Membrane Lipidome}

Lipid molecules are composed of a hydrophilic head group and a hydrophobic tail region. The constituent lipids of biological membranes differ from one another in head groups, constituent fatty acids and therefore their physical properties. ${ }^{12}$ The length of the fatty acid tail in lipid molecules of biological membrane typically varies from 14 to 24 carbons. The degree of saturation is the other parameter that might be different in lipid membranes. Having one saturated chain and one monoor polyunsaturated chain is common for lipids in the membrane structure. The totality of these lipids in cells is referred to as the cell lipidome. One of the main components of membrane lipids is glycerophospholipids or phosphoglycerides which have two fatty acid chains and a phosphate headgroup joint together by a glycerol moiety. The phosphate head group can have attachments of either one of the ethanolamine, choline, serine, glycerol, or inositol molecules. ${ }^{13,14}$ Of those, lipids that their headgroups contain the above-mentioned molecules are named as phosphatidylethanolamines (PEs), phosphatidylcholines (PCs), phosphatidylserines (PSs), 
phosphatidylglycerols (PGs), and phosphatidylinositols (PIs), respectively. ${ }^{13}$ Sphingomyelin is another type of membrane lipids which has a fatty acid chain, a phosphocholine headgroup with a sphingosine moiety. Glycerophospholipids together with sphingomyelin are known as phospholipids which have the ability to self-assemble when placed in a water-containing solution to form a bilayer membrane structure with the size of approximately $5 \mathrm{~nm} .{ }^{9}$ Other than phospholipids which are the main components of the bilayer membranes, glycolipids with a carbohydrate also exist in the bilayer membrane extending towards the extracellular environment. These molecules maintain the stability of the cell membrane and act as recognition sites which are crucial for cell to cell connection to form tissues. ${ }^{11}$ Sterols are another type of lipid molecules that are, along with phospholipids and glycolipids, extensively found in biological membranes. ${ }^{15,16}$

\subsubsection{Membrane Proteome}

In the same way, membrane proteome refers to the entire set of proteins in the cell's plasma membrane which are expressed by each given cell type at a given time. Membrane proteins are especially of importance because of the now well-known fact that different classes of proteins are expressed under different conditions. These differences are now regarded as proteomic signatures representing a new class of biomarkers. Since proteins are the main machinery of cellular function across all branches of life, their dysregulation has, therefore, been linked to numerous abnormal conditions. In addition, membrane proteins are responsible for many biological functions due to their role as receptors for the recognition of disease-causing organisms, hormones, and other signaling molecules. ${ }^{17}$ They play key roles in regulation of cellular activities and their function is essential for a wide range of physiological events such as neurotransmitter transport, adhesion, regulation and nerve impulse transmission.

Membrane proteins are classified into three main categories; 1) integral membrane proteins, 2) peripheral membrane proteins, and 3) polypeptide toxins. Integral membrane proteins or 
transmembrane proteins are permanently attached to the membrane and they are only detached using detergent or nonpolar solvents. On the other hand, peripheral membrane proteins are on the exterior part of the membrane i.e. surface and are temporarily attached to the lipid bilayer or a transmembrane protein via a hydrophobic, electrostatic and non-covalent interactions. Dissociation of these proteins take place using polar reagents such as an elevated $\mathrm{pH}$ or a high-salt concentration solution. The third group of membrane proteins consists of polypeptide toxins or pore-forming proteins and many antibacterial peptides. They are commonly considered cytotoxic since they create pores in the membrane and eventually cause lysis of the target cell. These proteins are mostly water-soluble; however, once aggregated, they can be irreversibly connected to the lipid bilayer.

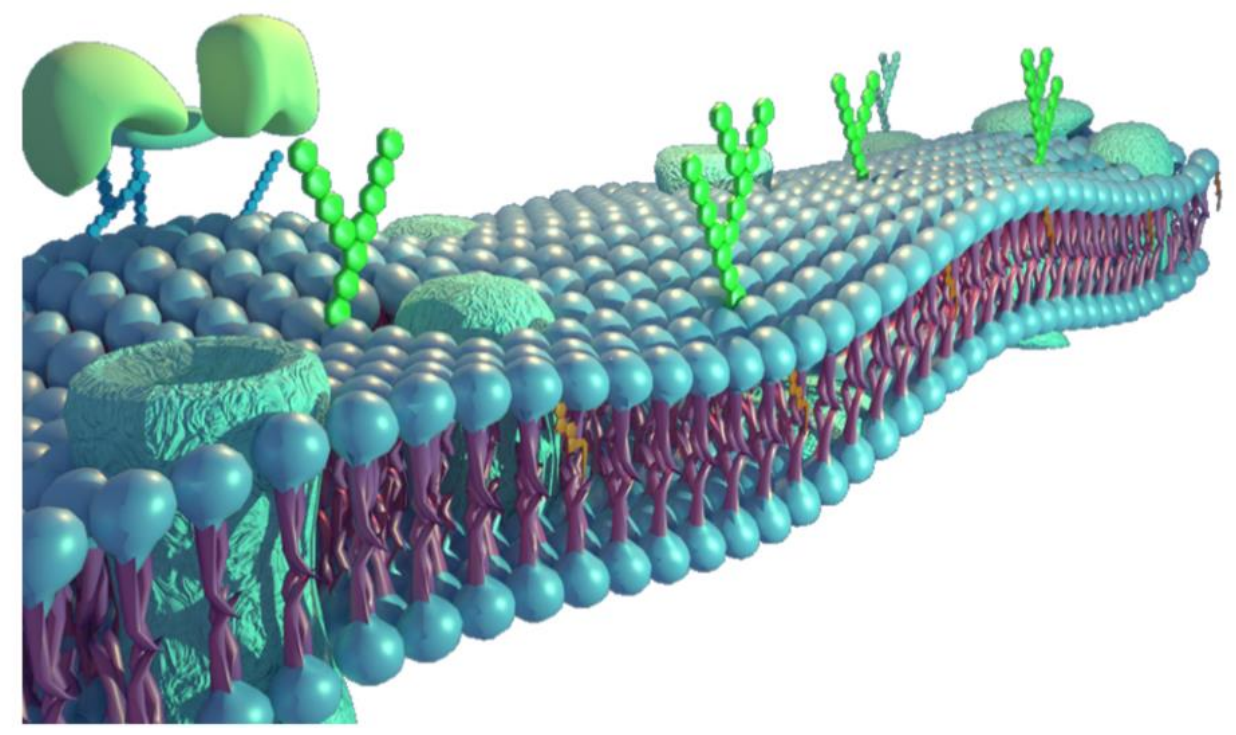

Figure 1-2 Schematic illustration of a biological membrane, showing a), the saturated and unsaturated lipid molecules, cholesterol, the actin network, glycolipids, glycosylphosphatidylinositol (GPI)anchored proteins, lipidated proteins, and transmembrane proteins. The subdomains enriched in saturated lipids, cholesterol, (GPI)-anchored and lipidated proteins are known as lipid rafts, characterized by their higher packing order and decreased fluidity compared to the non-raft domains. b) Uneven component distribution in each leaflet and among the inner and outer leaflets of a biological membrane leads to a membrane organization consisting of various small raft-like and non-raft domains. (Reprinted with permission from ${ }^{18}$ ). 


\subsubsection{Extracellular Vesicles}

Communication between cells is a vital function for multicellular organisms. This communication is either through cell-cell contact or transferring secreted molecules and extracellular vesicles (EVs). The secreted extracellular vesicles are originated either from plasma membrane or endosomal region of the cells. ${ }^{19}$ It has been well-established for a long time that during apoptosis

of a cell, particles known as apoptotic bodies would be released. However, the idea of releasing these EVs by healthy cells is somewhat new. ${ }^{19}$ In fact, the importance of EVs in influencing physiological and pathological conditions was not clear until recently. ${ }^{19}$ Their role includes, but is not limited to, transferring of genetic cargo and receptors and ultimately initiating pathways ${ }^{20}$, as well as modulating immune response and facilitating tissue regeneration. ${ }^{21}$ Besides their similarities, EVs are distinct based on their cellular origin, size, and role in intercellular communication and are classified into different categories such as microvesicles (MVs), exosomes, prostasomes, oncosomes, and apoptotic bodies, to name a few. ${ }^{19,22-24}$

Microvesicles (MVs) with the size in the range of 100-1000 nm are secreted from the plasma membranes of almost all cell types and can be found in bodily fluids and interstitial space between cells. They can transport mRNA, miRNA, and proteins between cells. MVs contain information about the antigenic content of their parent cells. MVs generated from tumor cells has been shown to carry genomic DNA (gDNA), cDNA and retrotransposon elements which can be utilized as a tumor biomarker ${ }^{25}$.

Exosomes are another type of EVs with the size range of 30-100 nm in diameter and density of 1.13 to $1.19 \mathrm{~g} / \mathrm{ml}$. They are shed either directly by the plasma membrane or during fusion of multivesicular bodies with the plasma membrane. The transferred information in exosomes and other types of EVs are encapsulated in a phospholipid bilayer membrane. This bilayer membrane 
in exosomes contains significant amounts of cholesterol, sphingomyelin, ceramide, and detergentresistant membrane domains (lipid rafts). ${ }^{26}$

Another type of specialty sub-micron membrane vesicle is prostasome with the size of $40-500 \mathrm{~nm}$ in diameter that is shed by prostate gland epithelial cells. Prostasome has a highly ordered structure and the composition of its membrane is similar to the one of lipid rafts. The role of prostasomes is to increase mobility of sperm and protect it against female immune defense during its passage to the egg. However, it has been shown that prostate cancer cells secrete prostasomes on a regular basis. As a result, the cancerous cells are protected due to the improved immune activities of prostasomes. ${ }^{27,28}$ Also of significance are another group of cancer-related vesicles known as oncosomes. The term "oncosome" was first introduced by Meehan et al. ${ }^{29}$ in a study to highlight the oncogene-carrying EVs secreted from (brain) tumors. The size of these vesicles is in the range of 100-400 $\mathrm{nm}$ and they contains phosphatidylserine on their membranes. ${ }^{29-32}$

Apoptotic bodies are the type of extracellular vesicles that are released when cells undergoes apoptosis. ${ }^{19,22-24,26}$ Apoptotic cells release two types of apoptotic bodies, one of which is initiated from the plasma membrane and contains DNA and histones while the other one is originated from the endoplasmic reticulum without any DNA or histones. The size of these particles is similar to that of platelets (1 to $5 \mathrm{um}$ ), which is larger than other types of EVs. Both apoptotic bodies and platelets express phosphatidylserine (PS) upon apoptosis. This makes it difficult to make a distinction between each type based on either size or PS expression. There is also a partial overlapping density between densities of exosomes with those of apoptotic bodies $(1.16-1.28$ $\mathrm{g} / \mathrm{ml})^{26}$ 


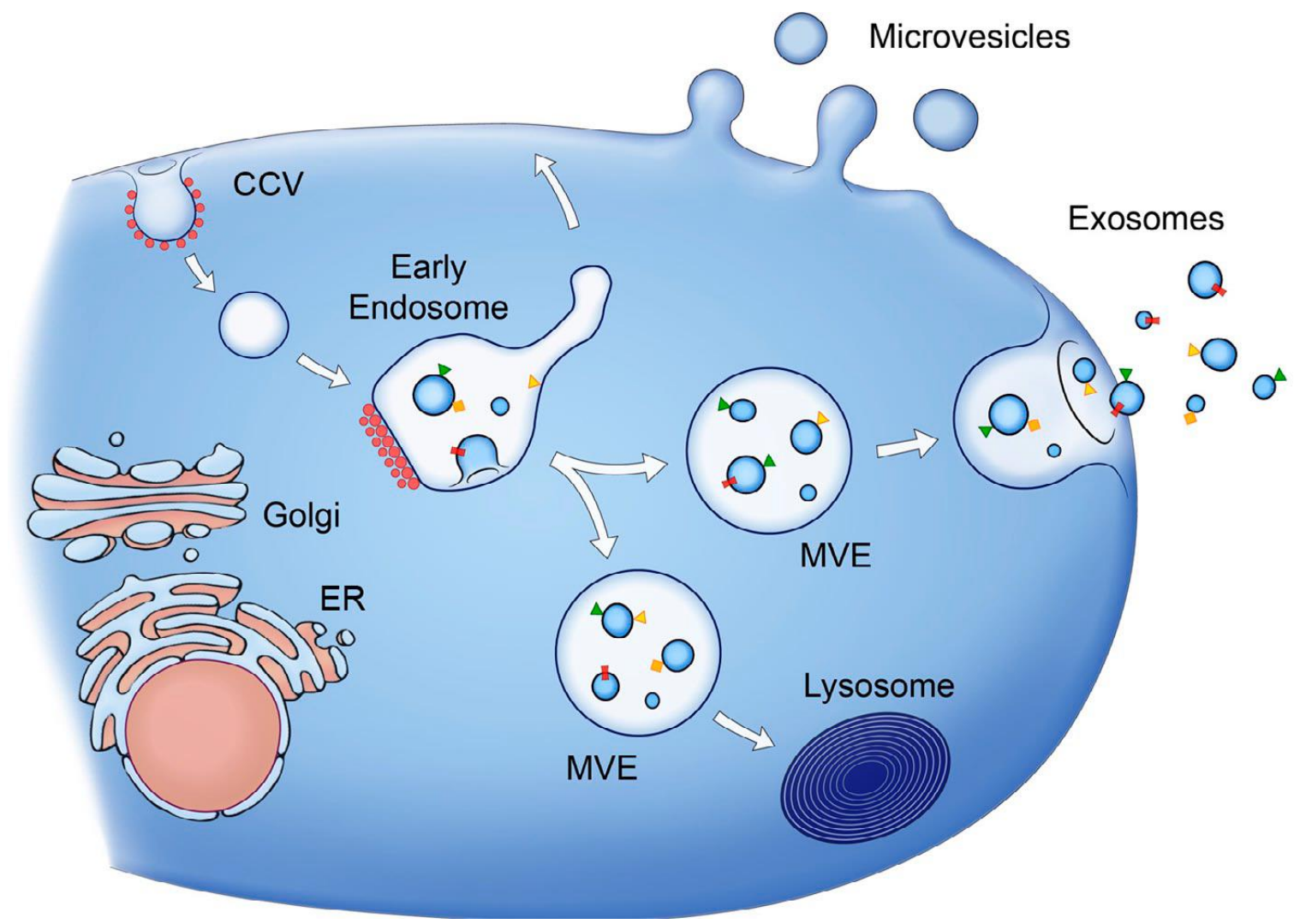

Figure 1-3 Formation of MVs and exosomes. MVs are shed directly from the membrane whereas exosomes are first formed by budding into early endosomes or multivesicular endosomes (MVEs) and then released in the extracellular environment through fusion of MVEs with plasma membrane (Reprinted with permission from ${ }^{19}$ ).

\subsubsection{Model Membranes}

Model membranes have been commonly used to study the biophysical properties of biological membranes and to investigate the effect of various components of cell membranes ${ }^{11}$, as well as in understanding of many physiological processes, such as signaling, cytolysis, formation of ion channels, and cellular recognition, which have many applications especially in developing biomedical and bioengineered products that exploit the interactions between peptides and lipid membranes. ${ }^{33}$ A case in point is the design of new antimicrobial peptides to fulfill the acute need for new sources of antimicrobial substances with the ascending trend of resistance of various bacteria to available antibiotic drugs ${ }^{34}$ However, the study of these proteins and peptides faces a 
number of challenges as they are involved in vital processes of cells and investigating their active form behavior without their lipid bilayer environment is impossible. ${ }^{35}$ Supported lipid bilayers (SLB) and vesicles are the most commonly used model systems. ${ }^{11}$ SLBs are lipid bilayers that are usually placed on the surface of a polished glass or mica surface. ${ }^{36,37}$ Vesicles can be made of lipids or other types of polymers or a composite of both. If vesicles are composed of a lipid bilayer they can also be named as liposomes. Liposomal vesicles are categorized into different classes based on their size; namely, small unilamellar vesicles (SUVs), large (LUVs), and giant unilamellar vesicles (GUVs). ${ }^{38,39}$ GUVs have been served as reliable model systems for real cells as their size (1-100 $\mu \mathrm{m})$ is in the same size range of most biological cells, their composition could be carefully controlled and their visualization is easily accomplished. ${ }^{38-40}$ Other types of vesicles are the ones that have more than one concentric bilayers and are called multilamellar vesicles (MLVs) and giant multilamellar vesicles (GMVs). ${ }^{38,39}$

\subsubsection{Elastic Deformations of Lipid Bilayers}

Biological membranes undergo various types of elastic deformation, four types of which are frequently used in model membranes to study the behavior of membranes and their embedded proteins. These modes of elastic deformation are the dilation or stretching, bending, shearing, and monolayer slipping which are schematically shown in Figure 1.4. The deformation of membrane via bending is studied in many bioengineering research areas in which the response of membrane to bending is characterized by the bending rigidity $\left(k_{c}\right)$ or the Gaussian curvature elastic modulus $\left(\bar{k}_{c}\right) .{ }^{11}$ The bending rigidity is also known as the mean curvature elastic modulus and is described as the gradient of mean bending moment per unit length of the bilayer $(\bar{M})$ to the mean curvature $(\bar{c}):^{13}$

$k_{c}=\left(\frac{\partial \bar{M}}{\partial \bar{c}}\right)_{A, \bar{c}_{G}}$ 
Where $\bar{M}=k_{c} \bar{c}$ and $\bar{c}=c_{1}+c_{2}$, i.e. the sum of the principal curvatures. The principal curvature axes is the inverse of the principal radii of curvatures $R_{1}$ and $R_{2}$ meaning: $c_{1}=1 / R_{1}$ and $c_{2}=$ $1 / R_{2} \cdot{ }^{13}$

Similarly, the Gaussian curvature elastic modulus is described as the gradient of Gaussian bending moment per unit length of the bilayer $\left(\bar{M}_{G}\right)$ to the Gaussian curvature $\left(\bar{c}_{G}\right)$ :

$$
\bar{k}_{c}=\left(\frac{\partial \bar{M}_{G}}{\partial \bar{c}_{G}}\right)_{A, \bar{c}}
$$

Where $\bar{M}_{G}=\bar{k}_{c} \bar{c}_{G}$ and the Gaussian curvature is $\bar{c}_{G}=c_{1} c_{2}$.

Conclusively, the total curvature elastic energy would be as follows:

$$
\Delta G_{C}\left(\bar{c}, \bar{c}_{G}\right)=\frac{1}{2} k_{c} A\left(\bar{c}-c_{0}\right)^{2}+\bar{k}_{c} A \bar{c}_{G}^{2}
$$

Where, $c_{0}$ is the intrinsic curvature of the membrane. Thermal fluctuation analysis of the membrane, ${ }^{41,42}$ micropipette aspiration studies ${ }^{43}$ and the analysis of the effect of curvature fluctuations on the XRD patterns of lipid bilayers ${ }^{44}$ are the methods that have been used to measure the bending rigidity of membranes.

\subsubsection{Effect of Molecular Structure}

The bending stiffness of a membrane is proportional to the square of the hydrophobic thickness of the lipid molecule:

$$
k_{c} \sim \frac{d_{h}^{2}}{a}
$$

In which $d_{h}$ is the hydrophobic thickness of the chain and $a$ is a constant value for the area per molecule of the lipids in the membrane. ${ }^{45}$ Figure 1.5 shows the correlation between the bilayer thickness $\left(D_{H H}\right)$ and $k_{c} / k T$ for various PC lipids. 


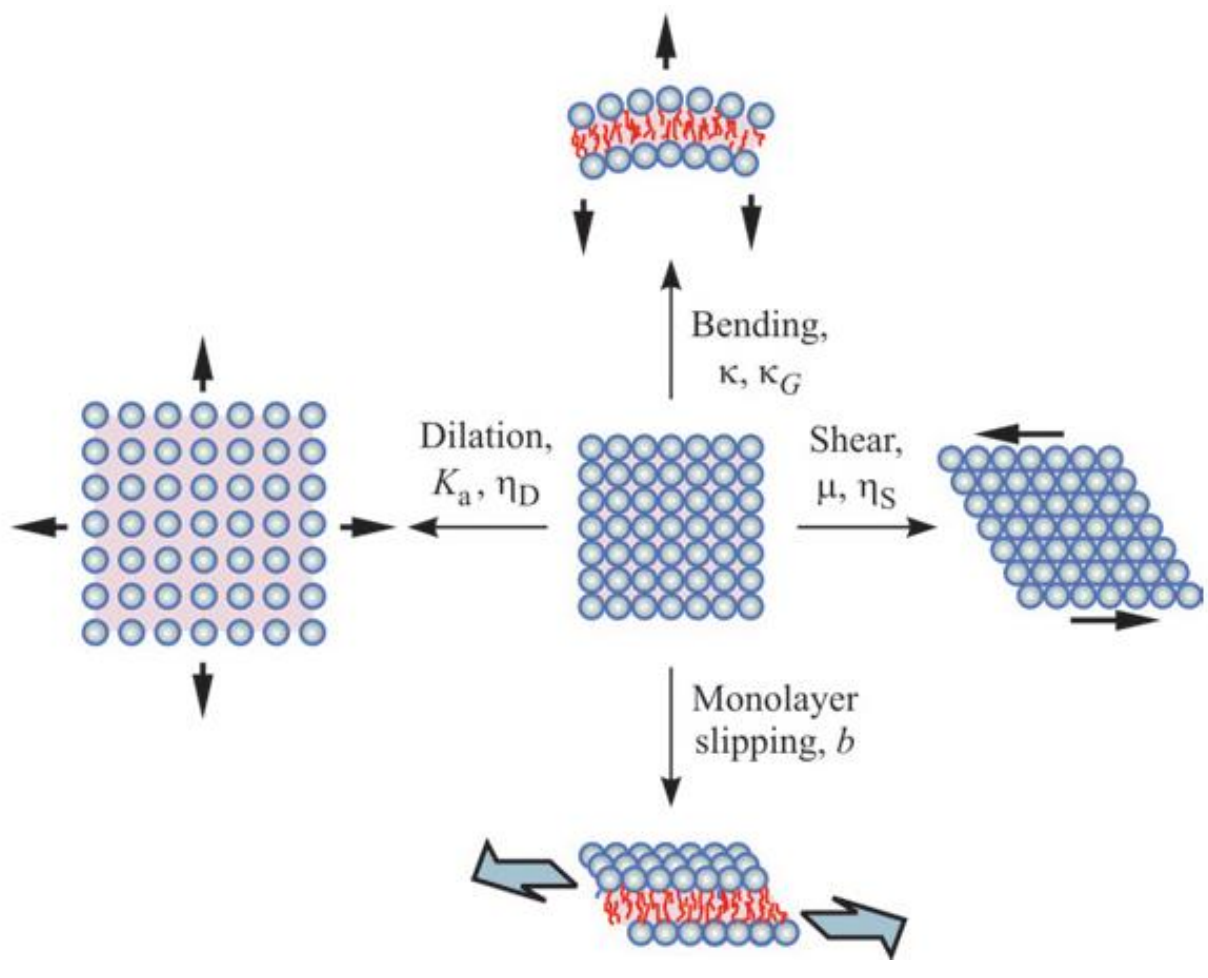

Figure 1-4 Schematic overview of modes of elastic deformation in bilayer membranes: Dilation or stretching, bending, shearing and monolayer slipping (Reprinted with permission from ${ }^{46}$ ).

\subsubsection{Effect of Temperature}

Temperature is the other parameter that has influence on the mechanical and physical properties of the membrane especially bending rigidity. ${ }^{11}$ Several studies focused on temperature-induced mechanical property changes and found out that there is a sharp drop in bending rigidity near the main phase transition temperature which is followed by a slight increase right after the transition. Following that, membrane experiences another small decrease and finally stabilization at temperatures few degrees higher than the main transition temperature. Overall, the drop in bending rigidity soften the membrane near the main transition temperature. ${ }^{45,47,48}$ 


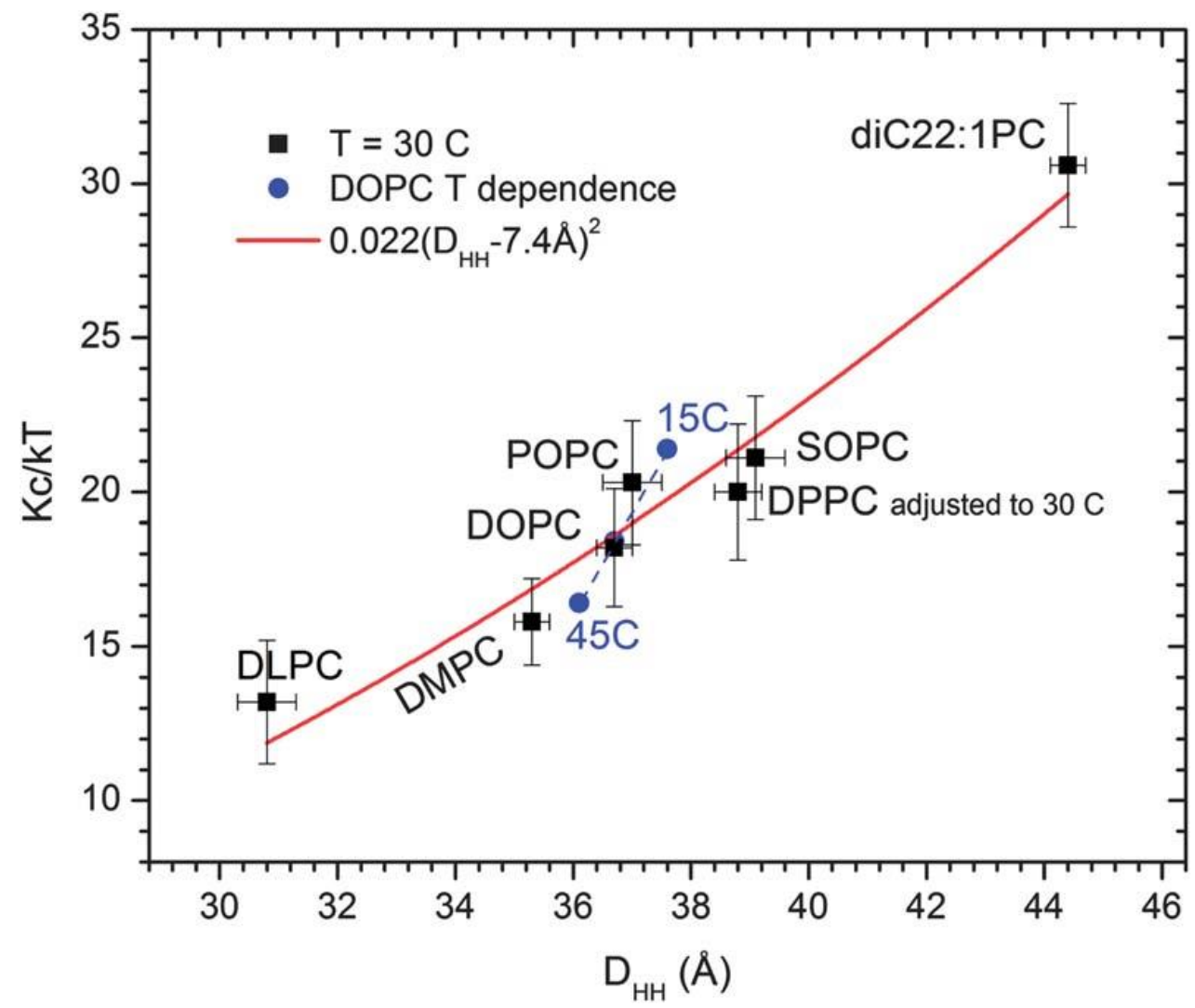

Figure 1-5 shows the dependence of $\mathrm{k}_{\mathrm{c}} / \mathrm{kT}_{\text {on }} \mathrm{D}_{\mathrm{HH}}$ for seven PC lipids. It also shows the effect of temperature on the bending rigidity for DOPC (Reprinted with permission from ${ }^{49}$ ).

\subsubsection{Effect of Other Molecules}

A biological membrane, other than lipid molecules, contains various other compounds which play a crucial role in determining mechanical properties of the membrane. Among these compounds are sterol molecules, proteins and peptides. The effect of proteins on the membrane mechanics has been investigated in various studies, some of which reported a significant increase in bending modulus of vesicles ${ }^{50,51}$, while others have shown either a neutral effect ${ }^{52}$ or a decrease in the membrane stiffness. ${ }^{53-56}$ Generally speaking, short peptides usually decrease the rigidity (stiffness) of the membrane ${ }^{54}$, while the effect of larger membrane proteins could be either neutral ${ }^{52}$ or decreasing. ${ }^{53,55}$ The reason for this is the hydrophobic mismatch due to the differences in the thickness of hydrophobic chain of the lipid molecule and the length of the hydrophobic surface of 
the protein ${ }^{57}$ that causes a local bilayer thinning in the case of a short peptide. The effect of proteins is discussed in more details in Chapter 4.

\subsection{Separation and Sorting of Particles Using Microfluidics}

\subsubsection{Overview}

Microfluidic technologies have recently experienced extensive progression in design, fabrication and experiments of miniaturized fluidic systems. ${ }^{58}$ The application of microfluidic devices includes biomedical, diagnostics, chemical analyses, automotive and electronics systems, among which the development of point-of-care diagnostic tools has gained a lot of attention. These diagnostic tools, also known as lab-on--a-chip (LOC) devices, are able to transport, prepare, separate, detect and analyze minute samples. The separation module has various applications in chemical synthesis, mineral processing and biological analysis. ${ }^{59,60}$ Increased application of microfluidic devices in diagnostic tools is mainly due to the recently known effect of several diseases in physical properties of cells which can be harnessed to reliably separate diseased cells from their healthy counterparts. ${ }^{61-}$

${ }^{63}$ Sorting and separation of cells as well as the fractionation of whole blood into its components have been discussed in various studies. ${ }^{64-67}$ Various types of microfluidic techniques are available to separate and sort particles. These methods can be classified into three main groups : 1) passive, 2) active and 3) combined techniques. In active methods, a foreign field is used to affect particles, whereas in passive method the interaction between the particles, microchannel and the flow field is used for separation. The combined method is most of the time a passive method employing one or more of the external fields used in active methods to further improve the efficiency. This chapter introduces some of the active, passive and combined techniques for sorting and separation of microand submicron-sized particle. 


\subsubsection{Passive Techniques}

\subsubsection{Pinched Flow Fractionation (PFF)}

Pinched flow fractionation (PFF) is a method of sorting particles based on their size. This method falls into passive techniques of separation as the separation take place using the laminar flow of the fluid. ${ }^{68}$ The schematic of such microchannel, as is shown in figure 1.6, includes a flow of fluid containing particles that is pinched by the flow of particle-free fluid in a "pinched segment". The pinched segment is where the particles are sorted at one of the sidewalls. These particles then tend to flow along the streamline pass through their center of mass. Depending on the size of the particle this streamline could be leaned towards or away of the wall of the microfluidic channel. Therefore a separation of particles based on their size is possible once they are flowing out of the pinched segment. ${ }^{69}$ The fluid in this technique assumes to be in steady-state condition, incompressible and has a low Reynolds number. Particles are also assumed not to cause perturbation in the fluid or interaction with the walls.

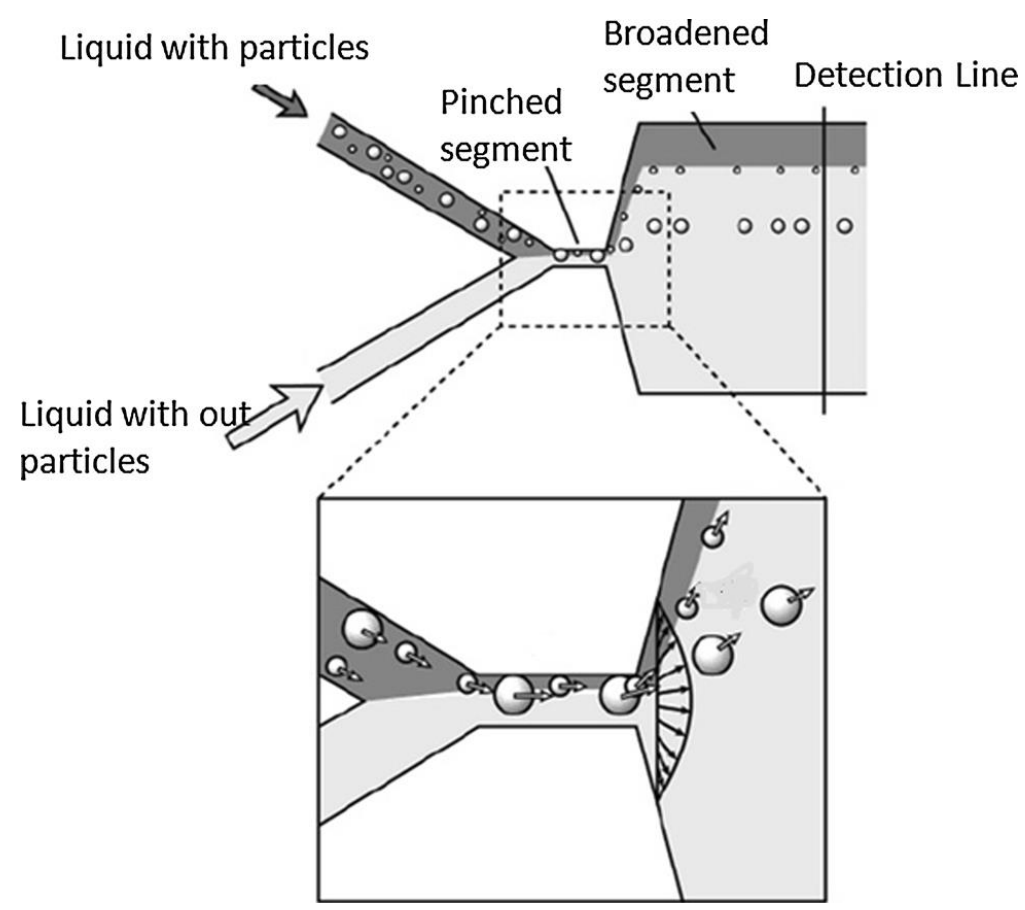

Figure 1-6 Schematic presentation of pinched flow fractionation (PFF) technique (Reprinted with permission from ${ }^{69}$ ) 


\subsubsection{Inertia and Dean Flow Fractionation}

The inertial lift forces that are existed on a particle in a straight microchannel are 1) shear-gradientinduced lift force, and 2) wall-effect-induced lift force. ${ }^{70}$ The shear-gradient-induced lift force is due to the differences in gradient of velocity in the centerline of the channel and the walls. Consequently, a particle moving along the channel close to the center line moves from the center towards the wall of the channel. ${ }^{71}$ The wall-effect-induced lift force is due to the formation of an asymmetric wake around the particle in the presence of a wall due to the flow field disturbance. In contrast to the shear-gradient-induced lift force, the wall-effect-induced lift force causes the particles to move away from the wall. ${ }^{72}$ The final lateral position of a particle depends on these two opposite-acting lift forces which affect the particle based on its size with respect to the size of the channel. ${ }^{73}$ The sorting based on size difference is therefore achieved with this method using an expansion region. In the expansion chamber (multi-orifice microchannel), the shear-gradientinduced lift force is dominant due to the size of this chamber and the long distance of the walls from the main stream. The particles in the main stream therefore move towards the walls as a result of the shear-gradient-induced lift force. These particles then pass through a series of expansioncontraction chambers in which there is the momentum change between the particles and the flow caused by the inertial lift forces and the induced lift forces. The position of the particle depends on the balance between initial force and drag force. ${ }^{70}$ The following equation shows the lateral migration velocity of the particle:

$$
U_{d}=\rho_{p} d^{2} U^{2} / 18 \mu D_{h}
$$

In which $\rho_{p}$ and $\mathrm{d}$, are the density and diameter of the particle respectively. $\mathrm{U}$ is the average velocity of the flow, $\mu$ is the viscosity of the fluid and $D_{h}$ is the hydraulic diameter of channel. As shown in this equation the lateral migration depends on the square of the diameter of the particle meaning 
that the smaller particles experience a smaller lateral shift than the larger particles leading to their separation. Figure 1.7 shows the schematic of a flow fractionation device with multi-orifice.

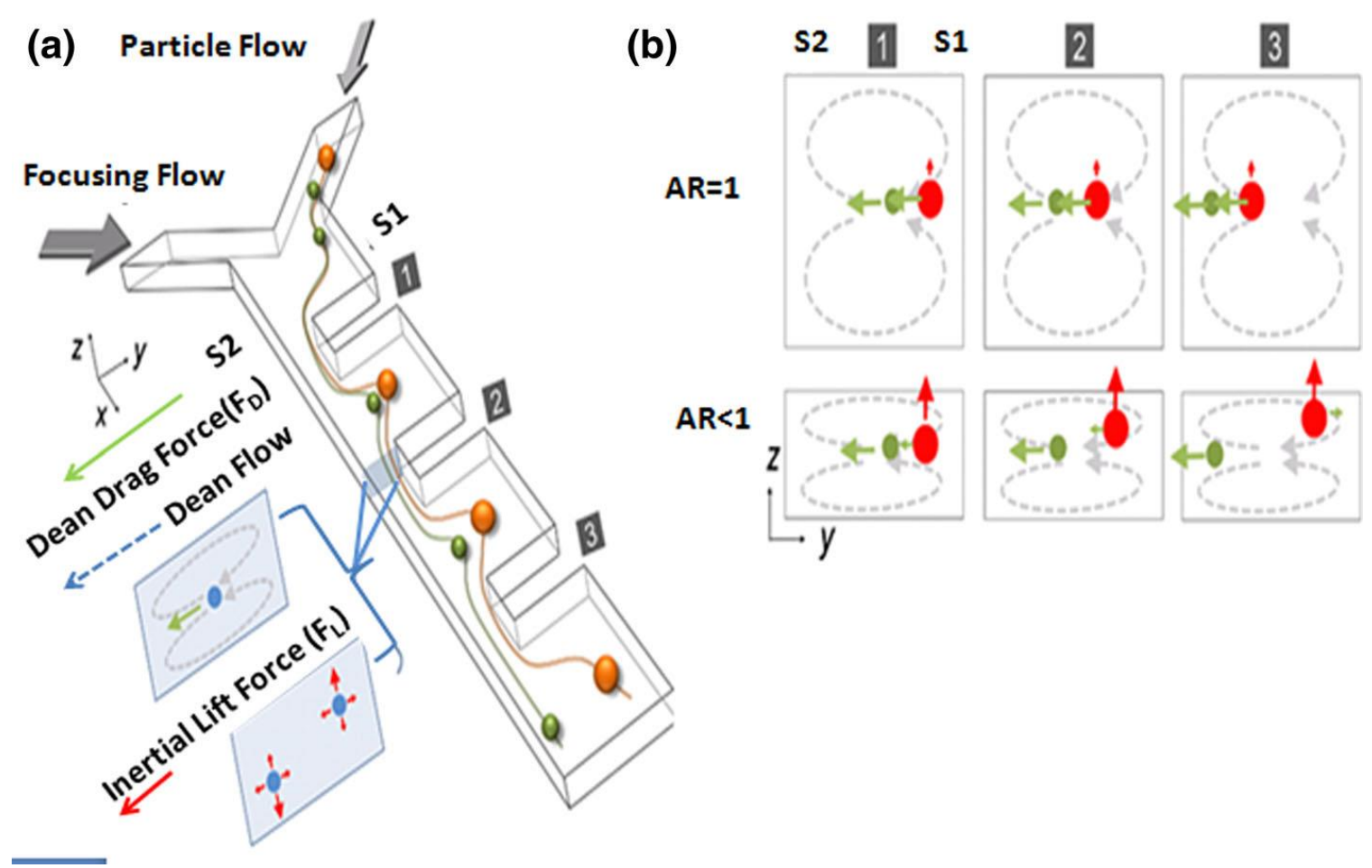

Figure 1-7 Schematic representation of a flow fractionation device (Reprinted with permission from ${ }^{74) \text {. }}$

\subsubsection{Microvortex Manipulation}

The separation of particles with microvortex manipulation (MVM) is based on the overall force resulting from gravitational force, buoyancy force and hydrodynamic drag force which cause a multi-stream condition in the channel. Figure 1.8 shows the separation of particles based on these multiple streams. The microfluidic channel is made such that there are multiple herringbone grooves at the bottom of the channel. Once the particle containing fluid is flowing over these grooves, a whirlpool shape flow is generated with an alternating direction for each groove. Particles that are lighter than the fluid migrate towards the top of the channel and position with regards to the balance of the interacting forces (upward buoyancy and downward drag and gravitational forces). Particles that are heavier than the medium sediment at the bottom of the channel. ${ }^{75}$ 

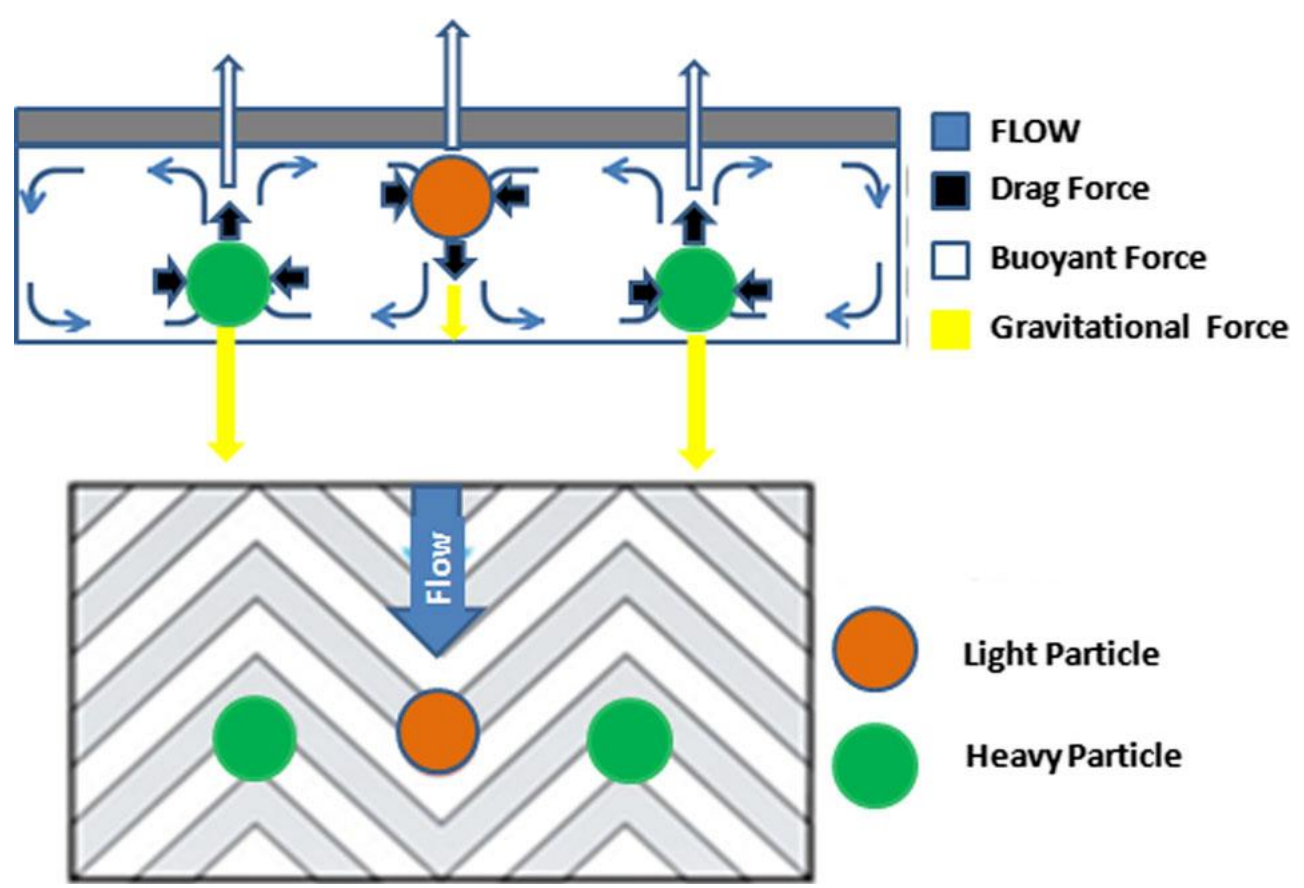

Figure 1-8 Schematic of particle manipulation in microvortex manipulation (MVM) technique (Reprinted with permission from ${ }^{75}$ ).

\subsubsection{Deterministic Lateral Displacement (DLD)}

In deterministic lateral displacement (DLD) method, the separation take place due to the asymmetric branching of the laminar flow. The microfluidic channel in this method is designed such that it has obstacles to navigate and separate particles based on their size and deformability. The gaps between the obstacles have to be larger than the size of the particles to be separated. ${ }^{76}$ There are two methods for the arrangement of the obstacles. The first method is the rotation of an array of obstacles, where in the second method a rhombic structure is used in the arrangement of obstacles. ${ }^{77,78}$ The lateral deviation of particles is caused by the irreversible interactions between obstacles and particles which lead to asymmetry of particles paths. The accumulation of each small lateral displacement of each obstacle results in a macroscopic deviation in migration direction. ${ }^{79}$ Sorting of particles using DLD method is shown in Figure 1.9. The mode of displacement of a particle depends on its size. There is a critical diameter of a particle $\left(D_{c}\right)$ which depends on the 
periods of the array $(\mathrm{N})$ and the diameter of each post (d) in the microfluidic channel and can be expressed by the following equation:

$D_{c}=2 \sqrt{(N / 3)}\left(\frac{d}{N}\right)$

A particle migrate along a zigzag path when the diameter of the particle is smaller than the critical diameter, whereas larger particles experience a lateral shifting depending on their size (displacement mode of migration). ${ }^{78,80}$ DLD method has been used for the separation of circulating tumor cells (CTCs) from normal cells ${ }^{81}$, sleeping parasites from blood ${ }^{82}$ and also for separating and sorting of droplets of different diameters ${ }^{83}$.

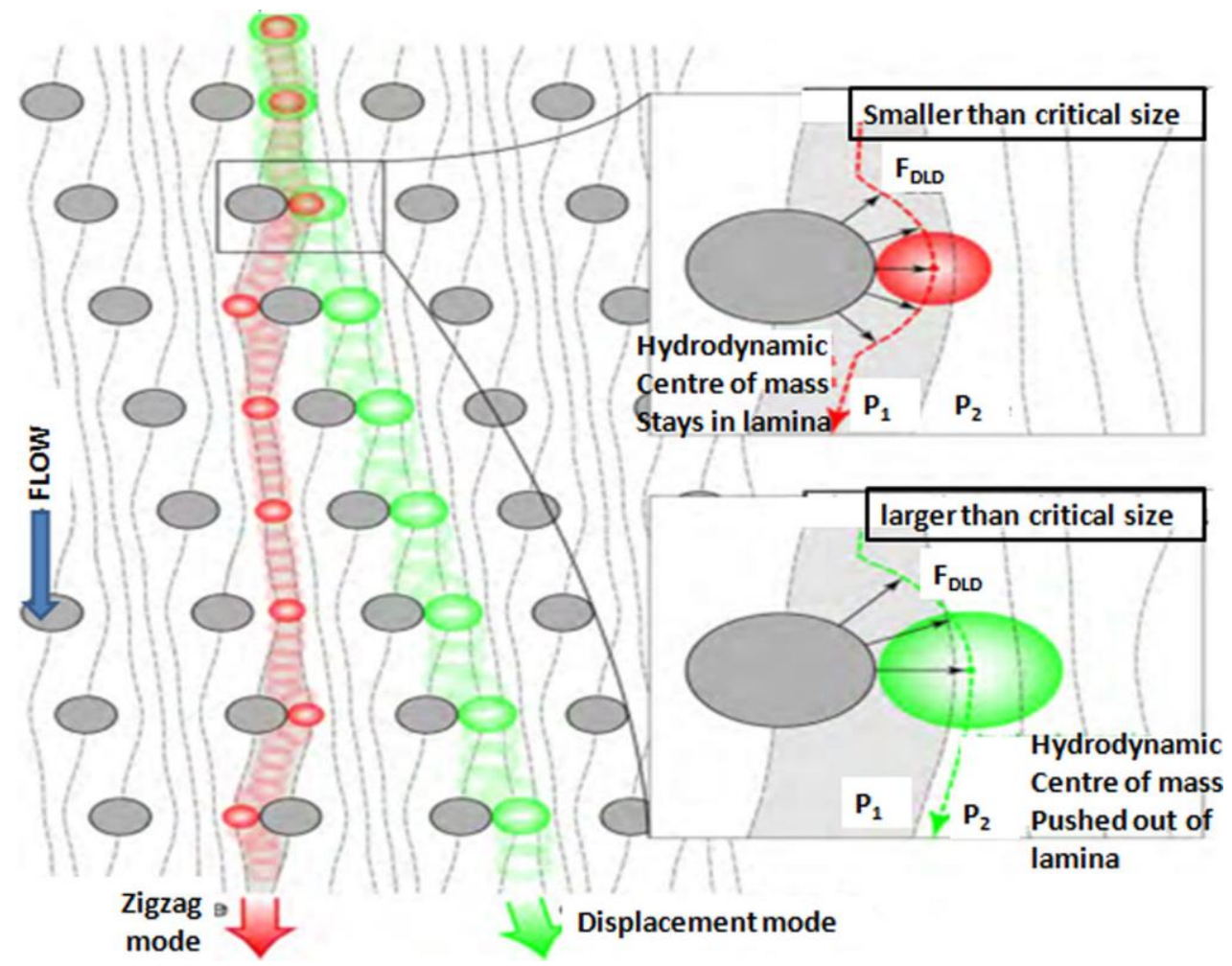

Figure 1-9 DLD sorting of particles through zigzag mode and displacement mode (Reprinted with permission from ${ }^{78}$ ). 


\subsubsection{Zweifach-Fung Effect}

Zweifach-Fung effect determines the tendency of particles to flow in the higher flow rate channel once they face a bifurcation. According to this effect, particles facing a bifurcated channel would flow through the high flow rate branch if: (I) the centroid of the particle is place beyond the critical streamline, or (III) the centroid of the particle is on the critical streamline. Otherwise, if (II) the centroid of the particle is within the critical streamline, the particle migrates into the low flow rate branch (Figure 1.10) ${ }^{84}$ There is a critical flow rate, above which all the particles migrate through the high flow rate channel. The critical flow rate ratio between two divided channels for particle to channel ratio in the order of one is $2.5: 1{ }^{84}$
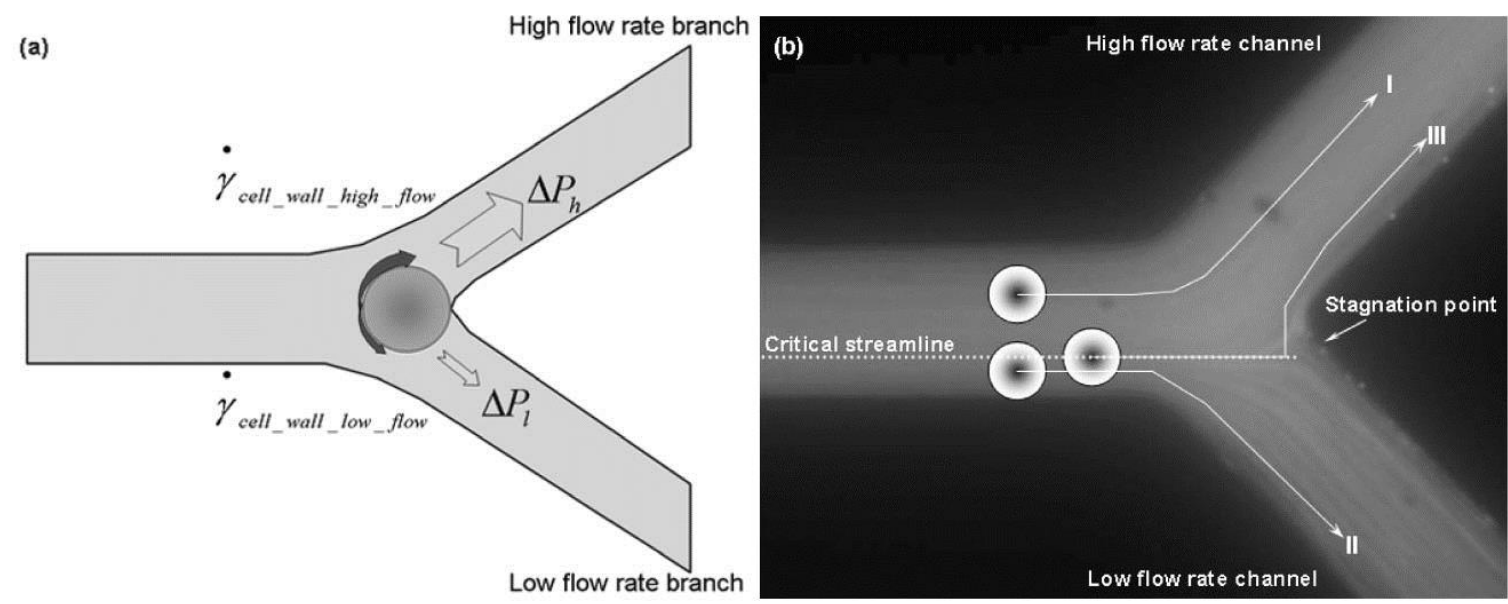

Figure 1-10 (a) Schematic illustration of Zweifach-Fung effect for the tendency of particles at a bifurcation. (b) Three different scenarios of particles migration at the bifurcation (Reprinted with permission from ${ }^{84}$ )

\subsubsection{Filtration}

Filtration is a method of separation of particles from sample based on their size. There are four types of microfabricated filters (Figure 1.11 and 1.12): membrane, weir, pillar and cross-flow. ${ }^{65}$ Although a separation via microfabricated membrane is possible for a wide range of applications, the accumulation of particles in the membrane could hinder the separation. A cleaning process containing a series of reverse flow could be applied to unclog the membrane. The time duration of forward and reverse flow could be set to an optimum value in order to give the maximum separation 
efficiency. Experiments have used a reverse time of 0.5 to $4 \mathrm{~s}$ for a forward flow of 1 to $40 \mathrm{~s} .{ }^{85} \mathrm{~A}$ microfiltration device with a single membrane has been used to separate plasma from whole blood. ${ }^{86}$ For the separation of multiple constituents of different size from a sample, a multimembrane microchannel is needed with each membrane having different pore size. ${ }^{87}$ Other than membranes, pillar type and weir type filtration are widely used for the separation of particles in a microfluidic channel. Their main application is however the separation of RBC and WBC from whole blood. The device for such separations using a weir type filtration usually contains an inlet for blood injection, two integral weirs of $26.5 \mu \mathrm{m}$ height and $20 \mu \mathrm{m}$ width, and two separate outlet one of which for $\mathrm{RBC}$ and the other one for $\mathrm{WBC}$. The serpentine structure put two filtration barriers parallel to each other. The barriers in pillar type separation are pillars of $20 \mu \mathrm{m}$ diameter with gaps of $6.5 \mu \mathrm{m}$ fabricated in two rows. The larger WBC are filtered through these barriers while RBC can flow through them. The separation of WBC from RBC using this weir type filtration is more efficient than pillar type because of the small pillar gap. ${ }^{88}$ If the flow direction is perpendicular to the micropost array of weirs, it is called cross-flow filtration which has the same function as weir type and pillar type filtration and is used to separate blood components based on their size. It can also separate plasma from the blood. ${ }^{89}$ Other modification are also available to the filtration method which utilize capillary action ${ }^{90}$ or perfusion $^{91}$ to isolate particles and components of whole blood.
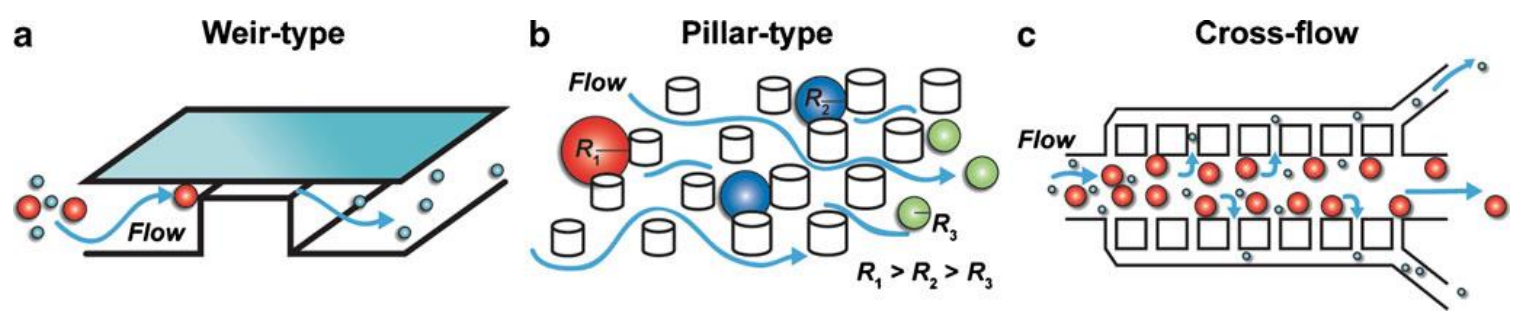

Figure 1-11 Schematic of microfilter designs, a) Weir type filter which enables the separation of particles based on size, b) Pillar type filter which contains pillar with designed spacing in order to hinder the passage of large particles, c) Cross-flow filters in which the flow direction is perpendicular to the array of pillars (Reprinted with permission from ${ }^{65}$ ) 


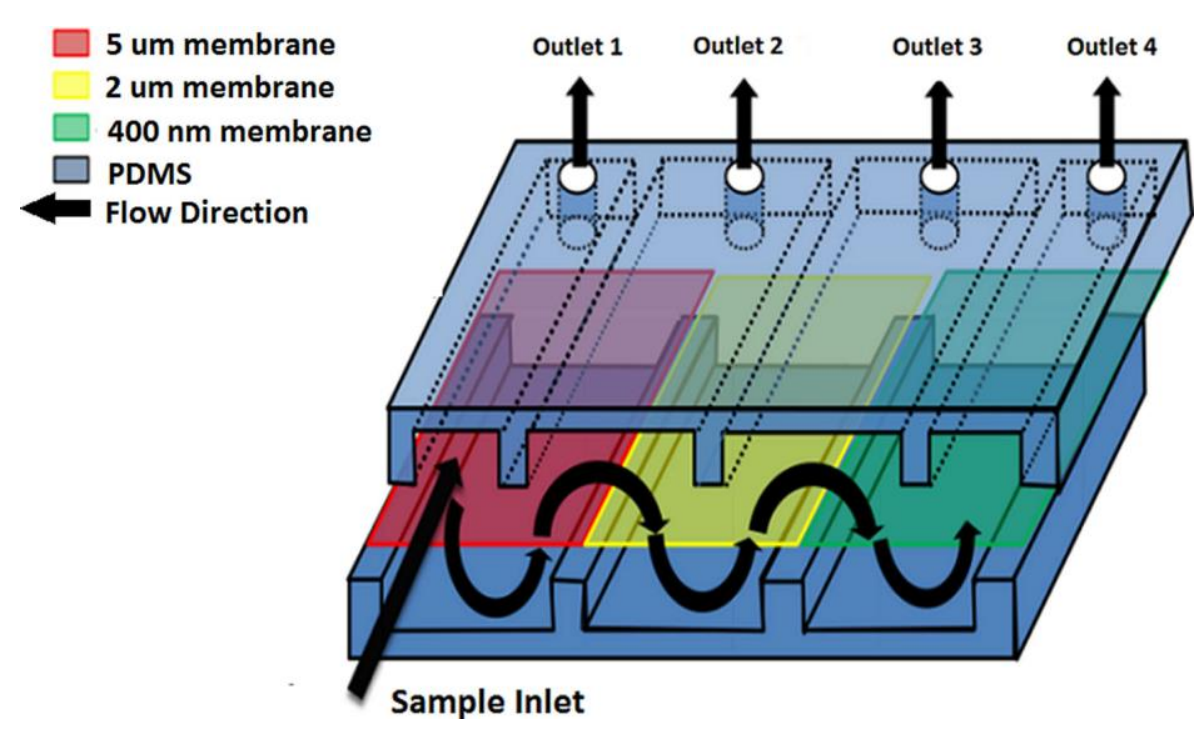

Figure 1-12 Schematic of microfilter design with multi membrane structure each of which has a different pore size (Reprinted with permission from ${ }^{87}$ ).

\subsubsection{Hydrodynamic Filtration}

Hydrodynamic filtration has side channels design similar to cross-flow filtration method. The fundamental of the work is however different in hydrodynamic filtration. In cross-flow filtration the side channels are used to collect only fluid not particles, whereas in hydrodynamic filtration these side channels are intended to collect the particles. The minimum size of the particles that are able to pass through a side channel depends on the volume flow rate of that side channel. Particles with the size of larger than a known value are not able to pass through the side channel if the volume flow rate of the channel is small. As shown in Figure 1.13, increasing the flow rate would increase the minimum size of the particles that can flow inside the side channel. ${ }^{92}$ This type of separation devices has been improved with the application of splitting and recombination of fluid using side channels. Using this technique, the fluid containing particles with smaller size is reinserted into the main channel and will flow mostly in the center of the main channel. Therefore, there is a separation of larger particles at the side walls and smaller particles flowing in the center. ${ }^{93}$ 


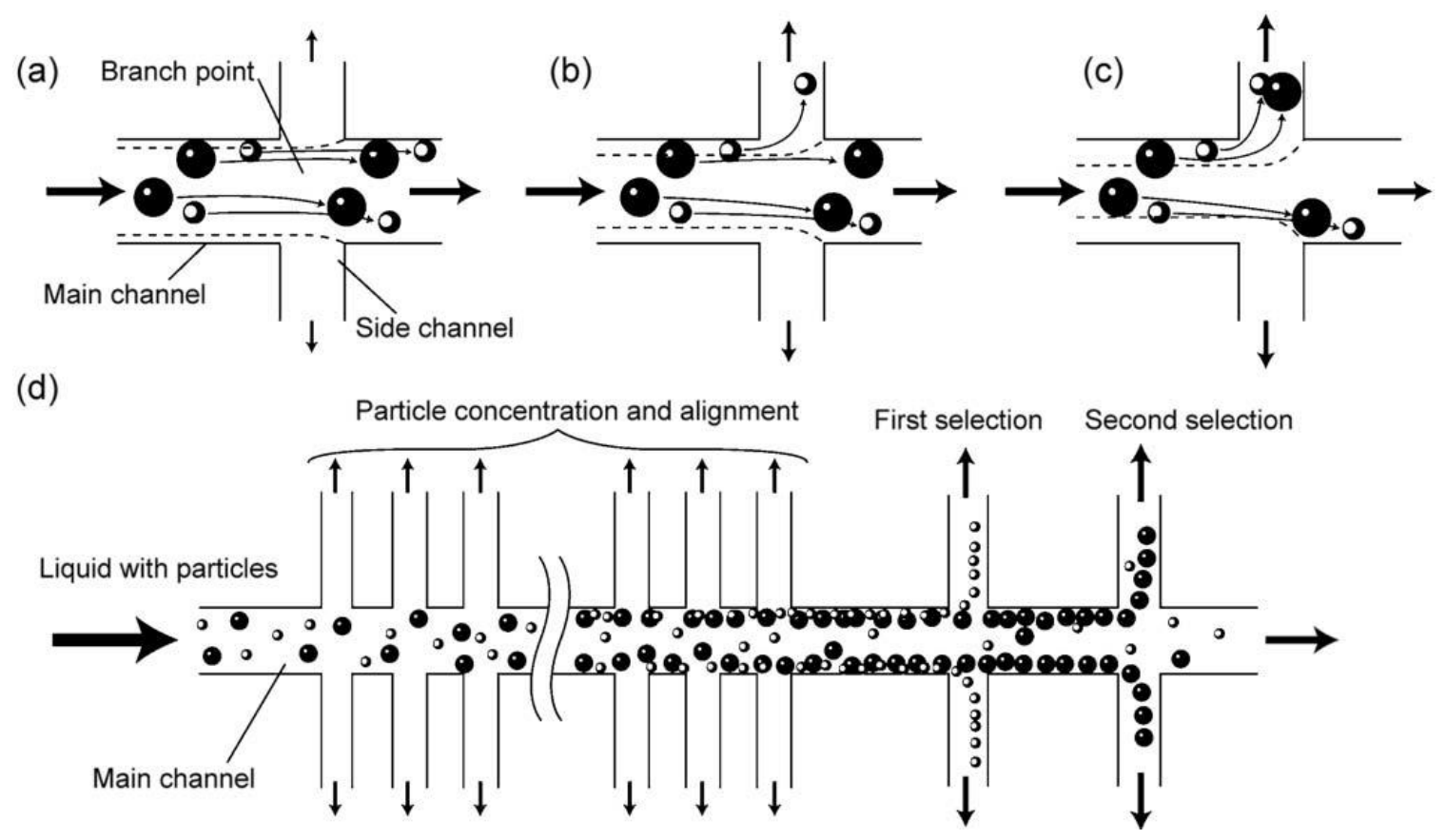

Figure 1-13 Fundamental of hydrodynamic filtration showing a separation capability depending on volume flow rate: (a) side channel has a small volume flow rate therefore particles will not pass through the side channel, (b) the volume flow rate is medium, therefore the side channel only allows the smaller size of the particles and particles with the size larger than a critical value are not able to pass into the side channel, (c) the side channel has the high volume flow rate, therefore the minimum size of the particles that can flow through the channel increased. (d) Schematic design of a microchannel for sorting and separation of multiple particles (Reprinted with permission from ${ }^{92}$ ).

\subsubsection{Micro-Hydrocyclone}

Micro-hydrocyclone is a method of separation particles from liquid with a spiral flow of fluid into a cylindrical chamber. This device as shown in Figure 1.14 is composed of an inlet tangential to the chamber, a cylindrical chamber with a frusto-conical bottom which is the main component of the device connected to the bottom outlet microchannel leading to a top outlet. The tangential injection of the fluid into the cyclone produce a helical flow inside the chamber which results in the separation of particles from the fluid based on centrifuge force. Radial velocity of particles depends on the density of the particles and fluid. The particles with density higher/lower than the density of the fluid can migrated away/ toward the center of the cylinder. For most chemical and biological samples the density of particles is higher than the density of the carrier fluid, so the particles are accumulated at the wall and separated. ${ }^{94}$ 

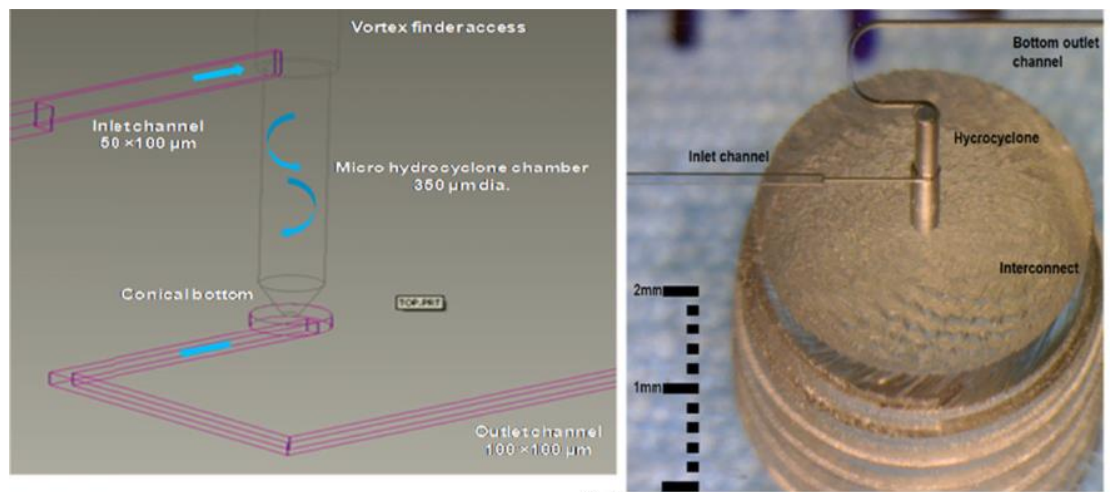

(a)

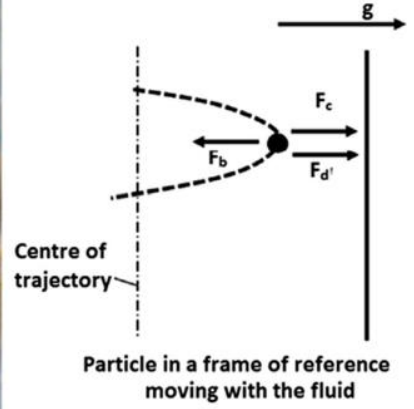

(b)

Figure 1-14 Schematic illustration of (a) a micro-hydrocyclone, and (b) forces acting on a particle in spiral flow inside the cylinder (Reprinted with permission from ${ }^{94}$ ).

\subsubsection{Active Techniques}

\subsubsection{Dielectrophoresis (DEP)}

A neutral particle in a non-uniform electric field will be polarized and affected by “dielectrophoresis (DEP)" force. This exerted force on a neutral particle in a homogeneous field is zero. However, DEP can maintain a non-zero value that has been used for trapping, focusing, translation, fractionation and characterization of particles. ${ }^{95}$ The time-averaged DEP force is expressed by the following equation:

$$
F(t)=2 \pi \varepsilon_{m} r^{3}\left\{\operatorname{Re}\left(f_{C M}(\omega)\right) \nabla E_{r m s}^{2}+\operatorname{Im}\left(f_{C M}(\omega)\right) \times\left(E_{x}^{2} \nabla \varphi_{x}+E_{y} \nabla \varphi_{y}+E_{z} \nabla \varphi_{z}\right)\right\}
$$

Where $r$ is the particle radius, $\omega$ is the angular frequency, $E_{R M S}$ is the root mean square value of the applied electric field, $\mathrm{E}_{\mathrm{i}}$ and $\varphi_{\mathrm{i}}(\mathrm{i}=\mathrm{x}, \mathrm{y}$, and $\mathrm{z})$ are the magnitude and phases of electric field components in the principal axis directions. $\operatorname{Re}\left(f_{C M}(\omega)\right)$ and $\operatorname{Im}\left(f_{C M}(\omega)\right)$ refers to real and imaginary parts of dipolar Clausius-Mossotti factor (CM) respectively. CM is parameter showing that a dipole moment is produced for a particle and its surrounding based on the frequency and could gain a positive or negative value. The real part of the equation determines the attraction or repulsion of particles to or away from the electrodes. When the $\mathrm{CM}$ is positive, particles are 
attracted towards the strong electric field. Otherwise, when the CM is negative, particles are attracted towards the weak electric field. ${ }^{95} \mathrm{CM}$ value can be calculated with the following equation:

$f_{C M}\left(\varepsilon_{P}^{*}, \varepsilon_{m}^{*}, \omega\right)=\frac{\varepsilon_{P}^{*}(\omega)-\varepsilon_{m}^{*}(\omega)}{\varepsilon_{P}^{*}(\omega)+2 \varepsilon_{m}^{*}(\omega)}$

Where, $\varepsilon_{P}^{*}$ and $\varepsilon_{m}^{*}$ are the frequency-dependent complex permittivity of particle and medium, respectively. For the $\mathrm{CM}$ to be positive, $\varepsilon_{P}^{*}$ should be larger than $\varepsilon_{m}^{*}$ and vice versa. The imaginary part of the equation determines the travelling field component which depends not only on the CM but also on the spatial nonuniformity of the field $\nabla \varphi_{i}$. This spatial nonuniformity shows the changes of electric field distribution with time. Depending on the sign of the imaginary part of $\mathrm{CM}, \nabla \varphi_{i}$ could act either in the same or opposite direction of the electric field ${ }^{95}$ Based on the equation, DEP force is proportional to $\mathrm{r}^{3}, \nabla E_{r m s}^{2}$ and dielectric properties of the particle. Since different particles in a medium could have various sizes and intrinsic properties, DEP could be applied to separate them. ${ }^{96}$ The dielectrophoretic properties of same size particles should be noticeably different in order to make a separation possible otherwise the target and nontarget particles are not separable. ${ }^{97}$

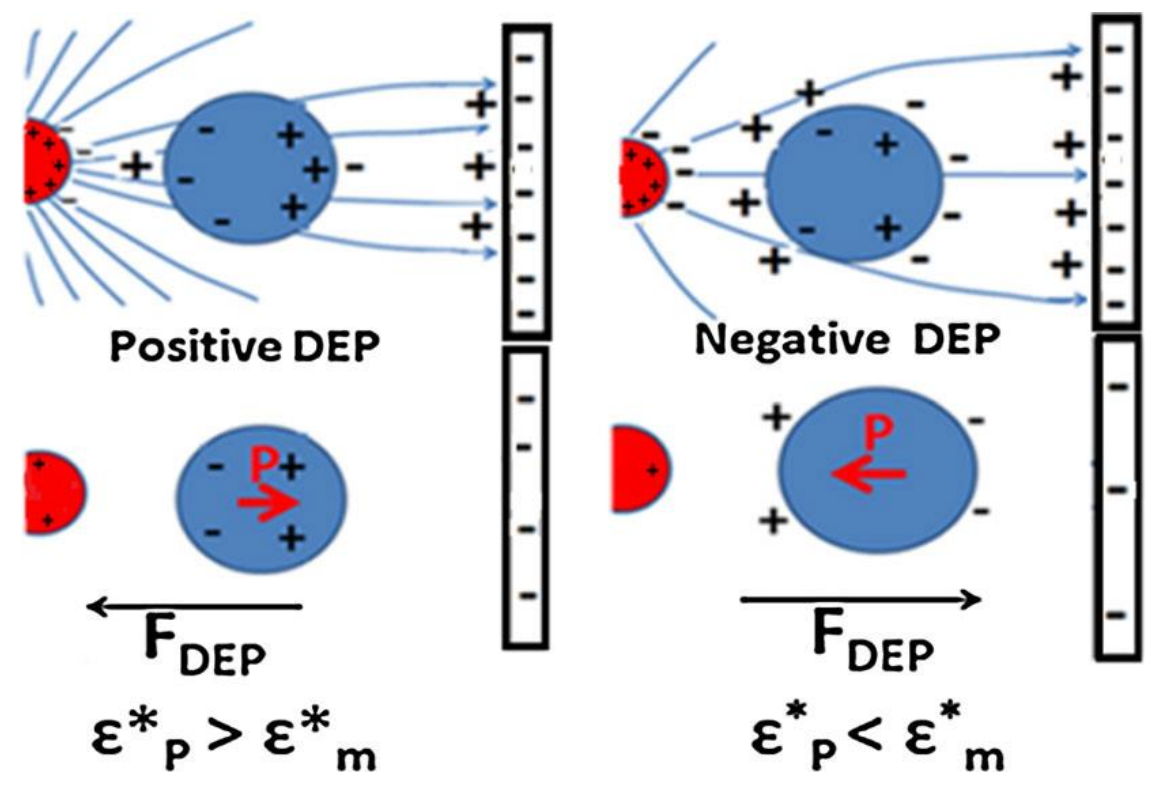

Figure 1-15 Schematic illustration of a nonuniform electric field with positive and negative DEP (Reprinted with permission from ${ }^{95}$ ). 


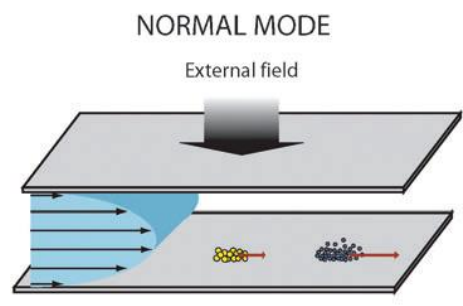

Particle size

- > .

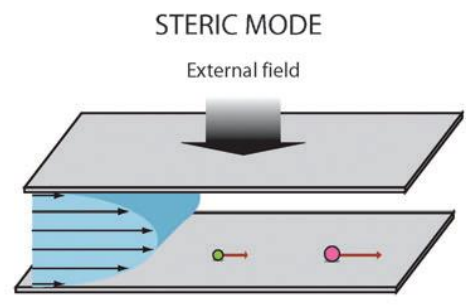

Particle size

$0<0$
HYPERLAYER MODE

External field

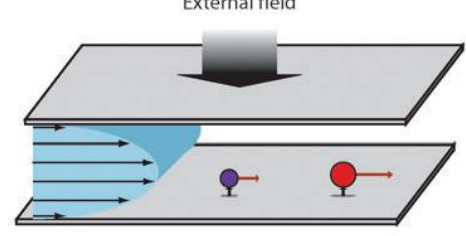

Particle size

$0<$

Figure 1-16 Showing the different modes of field flow fractionation (Reprinted with permission from 66 ).

To further improve the efficiency of the separation via DEP, a polymeric labelling bead is used to attach to the particles to be separated, for example cells. The significant difference between dielectrophoretic properties of labelled and unlabeled particles make the separation possible. This process is known as FACS, therefore, the separation of this type is called DEP-activated cell sorting (DACS).$^{97}$ Field flow fractionation (FFF) is one of the earliest methods to apply an electric field in microfluidic device for separation. ${ }^{66} \mathrm{FFF}$ has three modes of operation: a) normal mode, in which smaller particles are moved towards the walls in the direction of applied electric field, whereas the larger particles move away from the wall and concentrate in the center of the channel, b) steric mode, in which both small and large particles in the range of 0.5 to $10 \mathrm{~mm}$ migrate towards the wall and produce a thin film, however, larger particles move faster than smaller ones, and c) hyperlayer mode, in which the particles with the size larger than $10 \mathrm{~mm}$ is moved away from the wall due to hydrodynamic lift forces and larger particles collect from the outlet first as they move faster. Both steric and hyperlayer modes can be used with DEP where the frequency and voltage is controlled to have a better estimation of particle position.

\subsubsection{Magnetic}

Comparing to the above mentioned methods for separation, magnetic separation benefits from some advantages such as high specificity, inexpensive setup and relatively rapid separation. In addition, the viability of cells and biological samples are questionable when using particular separation 
technique. In filtration using a membrane, for instance, the applied pressure by the membrane may cause some damage. Also, the shear stress in centrifugation and heating in electrophoresis are the possible damage source to the cells. In magnetic separation however, the damage is minimize due to the short sorting time. ${ }^{98}$ Fluorescence-activated cell sorting (FACS) is another method of separation in which the efficiency is low due to the time consuming nature of the method $\left(10^{7}\right.$ particle per hour). ${ }^{99}$ Magnetic separation technique, which is the application of high-gradient magnetic field (HGMF), together with fluorescence labeling offers a more efficient separation. This method is called magnetic cell separation system (MACS). In conventional MACS the separation is achieved based on the existence or lack of the magnetization, therefore, it is not capable of sorting and separation of multiple particles at the same time. To address this issue, a multi-target magneticactivated cell sorter (MT-MACS) is introduced (Figure 1.17). This method employs ferromagnetic strips (MFS) to produce magnetophoretic force combined with hydrodynamic force of the flow to act on different target cells with different labelling. In this method the device has two different separation zones, each of which is designed to separate particles that are more responsive to the specific magnetic force of that region. ${ }^{100}$ The separation of magnetic beads (MBs) from nonmagnetic particles has been achieved using a micropillar array (MPA) method which uses nickel-iron micropillars in a silicone channel. ${ }^{101}$

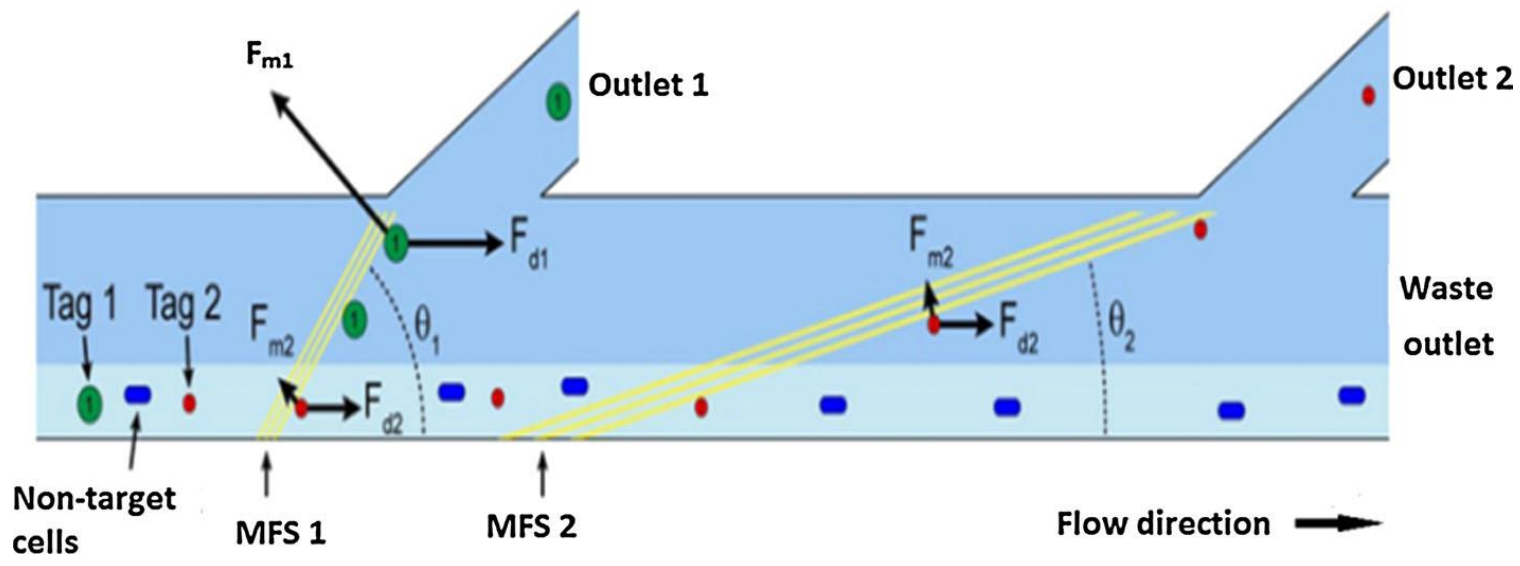

Figure 1-17 Schematic presentation of the application of a multitarget magnetic-activated cell sorter (MT-MACS) (Reprinted with permission from ${ }^{100}$ ). 
The magnetophoretic force exerting on a spherical particle in a nonuniform magnetic field is expressed by the following equation:

$$
F_{M A P}=2 \pi \mu_{0} f_{C M} a^{3} \nabla\left[H_{\text {ext }}\left(r_{0}\right)^{2}\right]
$$

Where, $f_{C M}$ is the Clausius-Mossotti factor of magnetization (CM) similar to dielectrophoresis. Diameter of the particle is shown as a, and $\nabla\left[H_{\text {ext }}\right]$ is the gradient of magnetic field. Here, CM can be calculated by the following equation:

$$
f_{C M}\left(\mu_{0}, \mu\right)=\frac{\mu-\mu_{0}}{\mu+2 \mu_{0}}
$$

In which $\mu$ is the magnetic permeability of the spherical particle and $\mu_{0}$ is the permeability of vacuum. Based on the equation, magnetophoretic force depends on the gradient of magnetic field, meaning that a nonuniform magnetic field is required to have a non-zero value for the force applied on a particle. ${ }^{102}$ The separation of magnetic from nonmagnetic particles as well as the separation of magnetic particles which have different sizes and magnetic susceptibility is also achieved using a free flow magnetophoresis. The device in Figure 1.18 shows a separation based on this method. This device uses several inlets and outlets to create a laminar flow inside the channel. The direction of magnetic field is perpendicular to the flow direction. The nonmagnetic particles are not affected by the magnetic field and continue to flow in their main path which guide them to towards one of the outlets. The magnetic particles, however, are affected by the magnetic field. Degree of deflection of particles depends on particle size and their susceptibility to magnetic field. Based on the forced applied to each particle, they will be guided to a separate outlet and can be collected from there. ${ }^{103}$ 


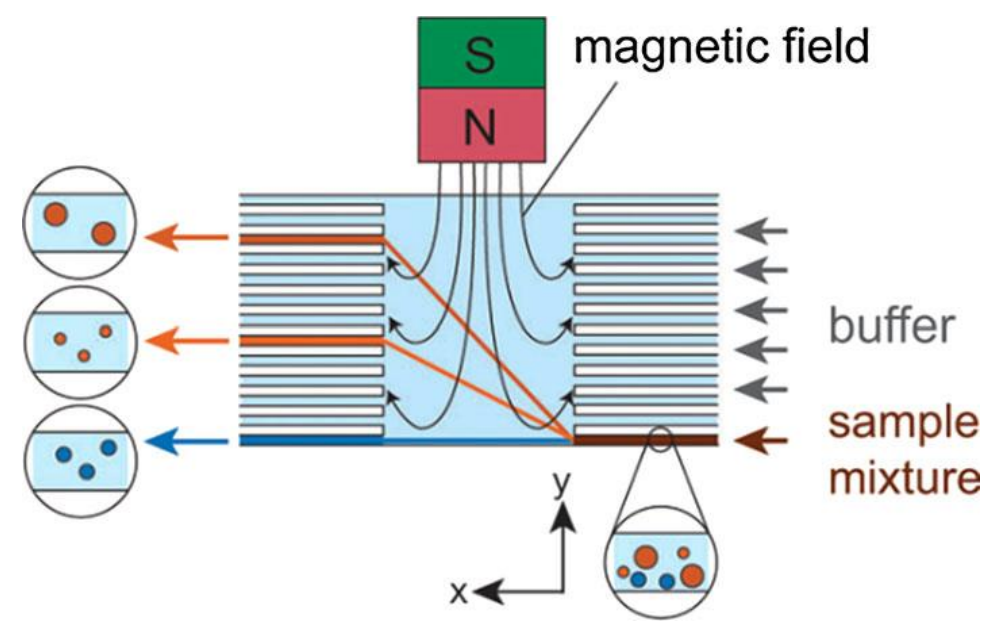

Figure 1-18 Schematic illustration of free-flow magnetophoresis for the separation of particles based on their size and magnetic properties (Reprinted with permission from ${ }^{103}$ ).

There is a magnetic method of separation similar to FFF which is called split-flow lateral-transport thin (SPLITT) separation. This method which is used frequently for preparation rather than analytical studies, consists of two inlets one of which for sample flow and the other one for buffer flow. Both fluids are injected through the chamber while the splitter separates them. Once the external field is activated the particles deflected to distinct distances due to their size difference. Figure 1.19 shows the separation of large (yellow) particles from the smaller (orange) particles using SPLITT method. ${ }^{66}$

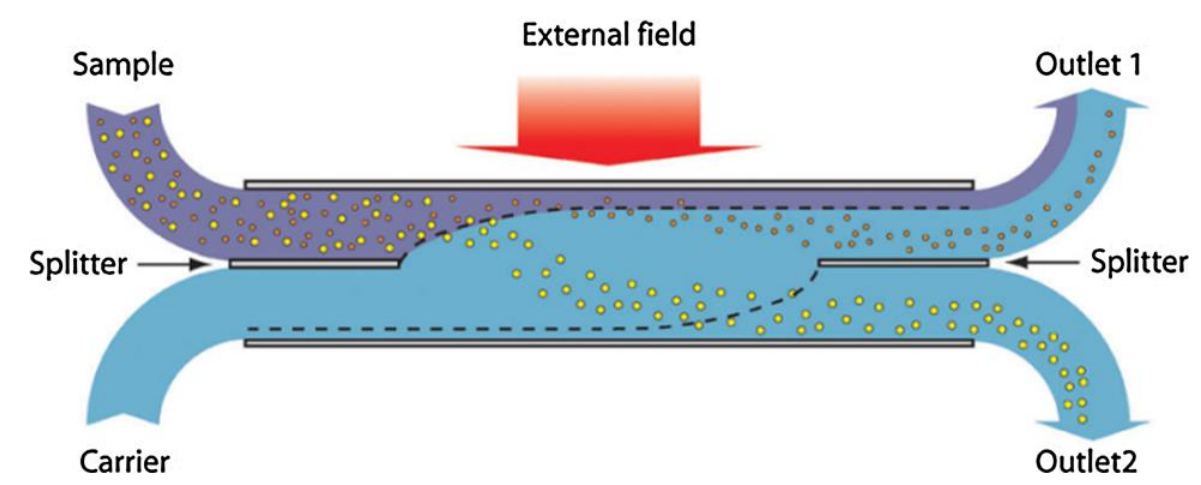

Figure 1-19 Schematic separation of particles using split-flow lateral-transport thin (SPLITT) (Reprinted with permission from ${ }^{66}$ ). 


\subsubsection{Optical}

Optical separation technique is based on the force produced by the scattered light beam since the momentum of it phonons is changed due to the incident and scattering. The intensity profile of a light beam is Gaussian with a maximum value at the center and decreasing trend towards the sides. Therefore, due to the scattering there is an imbalance of intensity across the beam which causes the application of scattering force on the particle and attracting it to the center of the beam. ${ }^{104}$ This phenomena is known as "single-beam gradient force trap for dielectric particles" or "optical tweezers". ${ }^{105,106}$ The wavelength and power of the laser source and its trap geometry can be manipulated to achieve separation of particles with different sizes ranging from $100 \mathrm{~nm}$ to 100 $\mu \mathrm{m} .{ }^{104}$ Optical trap lateral position is controlled by a steering mirror which adjusts the angle of the light going through the objective lens. If a single optical trap is used, the location of the trap and the trapped particles can be displaced with the movement of the laser beam or microscope stage. However, when a system contains multiple traps, "holographic optical tweezers" (HOTs) is used instead of steering mirror. HOT is in fact controls the movement of optical tweezers and creates traps for particles flowing inside a channel. The creation of optical traps is due to the diffraction of a single laser beam by a diffractive optic which is placed inclined to the direction of fluid flow. The movement of particles inside the traps are monitored with a camera. ${ }^{107}$ A modification to optical tweezers is the use of liquid crystal display (LCD) which enables the creation of arbitrary light fields for lateral trapping of freely moving particles in water. Since the LCD has a pixilated structure, the overall efficiency of this method is low. ${ }^{108}$ An optical lattice, which has the ability to control the amplitude and phase of each beam passing through it, is formed by the collimation of the beams which are split by passing a through a diffractive splitter. When a laminar flow of particles with different size and intrinsic properties pass through an optical lattice, the interaction of particles with optical lattice vary based on their properties. Less interactive particles deflect from the flow direction while more interactive particles follow the steamline path. Figure 1.20 shows an 
example of an optical lattice.[Gluckstad] Figure 1.20 (b) shows an example of the optical lattice with a body-centered tetragonal lattice structure. A buffer solution is injected from chamber A. The target particles are going to be collected into this buffer after flowing through the optical lattice from chamber B and deflecting toward the chamber C. In the absence of optical actuation, all particles flow their path from chamber B to D, however, in the presence of optical actuation, smaller particles that are less interactive with optical energy are deflected toward chamber $\mathrm{C}$ and larger particles move toward chamber D. ${ }^{109}$ There are methods to control spatial position of the optical beam. Acousto-optic deflector (AOD) is a conventional method and HOT is the more complicated new method to control the trap arrays location in two and three dimensions.

However, HOT cause extension on some of biomolecules such as DNA strand due to high spring constant. To study these molecules that should be analyzed in a free extension condition and under constant force another method is developed known as "asymmetric scanning line optical tweezer" (LOT). ${ }^{110}$ LOT takes advantage of an asymmetric beam profile in the back-focal plane of the microscope objective. Doing so will exert a small constant lateral radiation pressure force on the particle in a distance of about several micro meter.

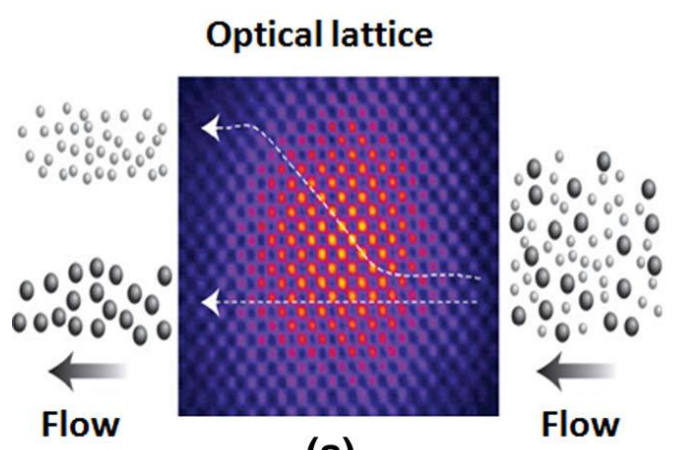

(a)

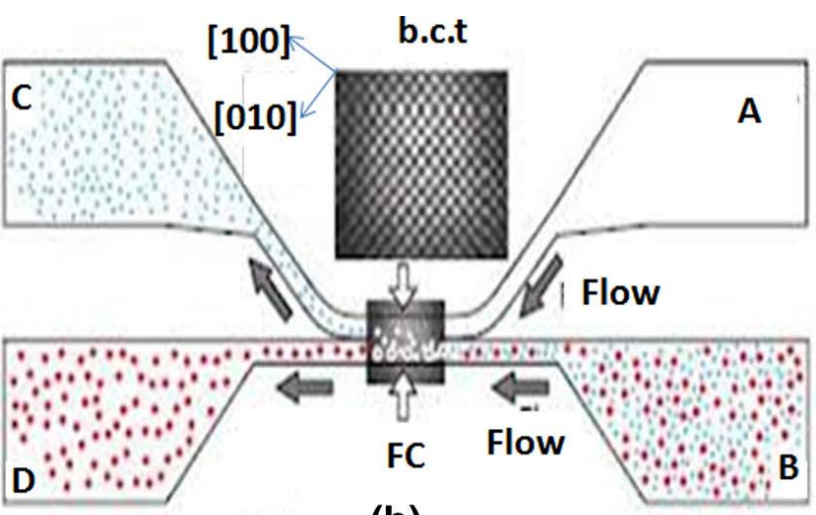

(b)

Figure 1-20 (a) optical lattice principles, and (b) Separation of particles using an optical lattice (Reprinted with permission from ${ }^{109}$ ). 


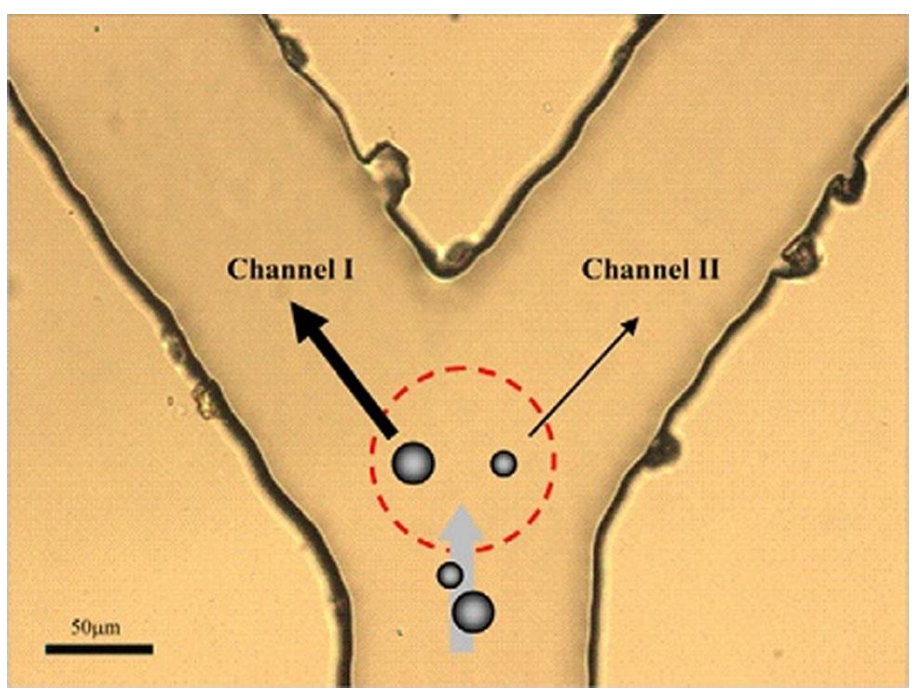

Figure 1-21 Optical micrograph of an LOT microfluidic device (Reprinted with permission from ${ }^{111}$ ).

IF there is two optical traps each of which has a specific wavelength and intensity distribution, then they are able to navigate particles in specific direction (two distinct LOTs). This could be done by two cylindrical lenses. ${ }^{111}$ Figure 1.21 shows the microscopic image of an LOT microfluidic chip.Other methods for sorting and counting of particles are optically induced flow cytometry, which is actually based on optically induced electrophoresis (ODEP) $)^{112}$ and optical chromatography (OC), which works mostly based on the resulting force of the balance between microfluidic drag force and optical radiation force. ${ }^{113}$ separation of particles using "optical tweezers" is based on the entrapment of particles towards the center of the beam by optical gradient force. This usually happens with the use of a lens to focus the light beam. Scattered beams are also used for separation and sorting particles. In this case, the displacement of particles is due to the scattering force of radiation and is known as "photophoresis". ${ }^{114}$ the direction of particle movement depends on the light absorptive properties of the particle. When a particle suspended in a fluid is hit by a scattered light beam, the particle will absorb the light and generate a nonuniform heat flux all over the particle volume. This nonuniformity of heat distribution results in the production of "photophoresis" force. If the particle has a high light absorptivity, the applied photophoresis force 
is called "positive photophoresis" and its direction guide the particle away from the light source. In contrast, if the particle has a low light absorptivity, the applied photophoresis force is called "negative photophoresis" and its direction guide the particle toward the light source. ${ }^{115}$ The separation efficiency using photophoretic force is increased with the size of particles. ${ }^{114}$ the photophoresis velocity depends on the size of the particles or size of the liquid molecules where increasing either of these sizes cause decreasing the photophoretic velocity. "16 "Thermophoresis" is another term used when a temperature gradient exists in the medium. This temperature gradient causes the particles to diffuse from high temperature regions to low temperature regions. Thermophoresis is used to separation droplets when the nonuniform heat distribution results in a temperature gradient in front and backside of a droplet. ${ }^{114}$ This heat generation and its uneven distribution is more evident in smaller particles and droplets, therefore, thermophoretic force is greater in smaller droplets. Also, thermophoresis and photophoresis are mixed to separate particles in water purification. ${ }^{115}$

\subsubsection{Acoustic}

Acoustic forces which is also called as "acoustophoresis" are the forces exerted on a particle in the media. These acoustic waves can be used to separate particles based on their physical properties such as density or compressibility.[Lenshof 2012] In an acoustic standing waves there are regions of high and low pressure which are called as pressure nodes and pressure antinodes. Most particles and cells are attracted to pressure nodes. The particles can be trapped, concentrated and separated based on the differences in their intrinsic physical properties. Similar to DEP, the force exerted on particles can be positive or negative based on their properties. ${ }^{117}$ [Bruus] Fundamentals of acoustophoresis and the related separation techniques are discussed in more details in the next chapter, since this method of separation is the basis upon which this work has been founded. 


\subsubsection{Combined Techniques}

Combined techniques are in fact passive separation techniques that use external fields. For example, a pinched flow fractionation (PFF) is combined with tunable electro-osmotic flow (EOF) for improving the size separation of the particles and is called as tunable PFF. ${ }^{118}$ In another combination method, PFF is combined with photophoresis, which is called optically enhanced pinched flow fractionation (OEPFF). In this method, when the particles passed through the pinched region, a laser beam is exposed on the particles which improves the efficiency of their size-based separation. ${ }^{119}$ Deterministic lateral displacement (DLD) can be combined with DEP to improve sorting efficiency. ${ }^{120}$

\subsection{Acoustophoresis}

\subsubsection{Overview}

The acoustic radiation force or acoustophoresis is the force exerted on a suspended particle in a liquid media in a presence of an acoustic radiation field. These forces are stronger when the ultrasonic frequency is adjusted to form standing waves, therefore, most of the acoustic separation

techniques are performed in this mode. ${ }^{121-123}$ A standing wave can acquire various geometries based on which the pressure node would be in the form of point, line or plane. The geometry of pressure nodes determines the position of particles in a standing wave acoustophoresis, ${ }^{124,125}$ therefore, enabling agglomeration or washing particles from a carrier fluid to another or fractionation and separation of them into different subpopulations. ${ }^{126-128}$ In this chapter the fundamentals and applications of acoustically-actuated microfluidic systems, which is also called "acoustofluidic systems", are discussed. In these systems, the manipulation and separation of particles are achieved with the application of acoustic radiation fields. 


\subsubsection{Standing-Waves}

In a standing wave, as introduced by Michael Faraday in 1831, each point has a constant amplitude due to either interference of two waves moving in opposite directions or movement of the medium in an opposite direction with respect to the wave. A common method to create a standing wave is to use a resonator. The interference of reflecting waves from the end of the medium with the incident waves coming out of the source if adjusted to the correct wavelength/frequencies forms the standing wave. These frequencies are known as harmonic frequencies or harmonics. Interferences in frequencies other than harmonics lead to non-repeating or irregular disturbances of the medium. The wave formed by the interference of two opposing waves can be expressed by the following equation:

$y(x, t)=A \cos \left(\frac{2 \pi x}{\lambda}+\frac{\phi}{2}\right) \sin \left(\omega t+\frac{\phi}{2}\right)$

In which, $y(x, t)$ is the displacement of a point in a standing wave at time $\mathrm{t}$ with respect to the longitudinal position $\mathrm{x}, \mathrm{A}$ is the amplitude of the wave, $\lambda$ is the wavelength, $\omega$ is the angular frequency, and $\Phi$ is the phase difference between two travelling waves. Based on this equation, pressure nodes are the positions where $\mathrm{x}$ is equal to the even multiples of $\frac{\lambda}{4}$ and the amplitude is zero. Similarly, anti-nodes are the positions where $\mathrm{x}$ is equal to the odd multiples of $\frac{\lambda}{4}$ and the amplitude is maximum in the standing wave.

\subsubsection{Acoustic Radiation Forces}

The gradient of acoustic potential is known as the acoustic radiation force and is expressed by the following equation: ${ }^{125}$

$F^{r a d}=-\nabla U^{r a d}$ 
In which it is assumed that the particles are spherical and their diameter is much smaller than the wavelength of the standing wave. $U^{\text {rad }}$ in this equation is the acoustic potential. The acoustic radiation force for a one-dimensional planar standing wave, in the $\mathrm{x}$ direction, can be written as the following equation:

$F_{x}^{r a d}=4 \pi \phi(\rho, \beta) k_{x} r^{3} E_{a c} \sin \left(2 k_{x} x\right)$

Where $\phi$ is the acoustic contrast factor, $k_{x}=2 \pi / \lambda$ is the wave vector in which $\lambda$ is the wavelength equal to $2 \mathrm{w}$ (the width of the channel times two), $r$ is the particle radius, $E_{a c}$ is the energy density and $\mathrm{x}$ is the distance. [11-ata] Based on this equation the acoustic radiation force exerted on a particle in a standing wave depends on the distance of the particle in the standing wave, energy density, particle size and acoustic contrast factor.

\subsubsection{Acoustic Contrast Factor}

Acoustic contrast factor $(\phi)$ can be expressed by the following equation:

$\phi(\rho, \beta)=\frac{1}{3}\left[\frac{5 \rho_{p}-2 \rho_{0}}{2 \rho_{p}+\rho_{0}}-\frac{\beta_{p}}{\beta_{0}}\right]$

Where $\rho_{p}$ and $\beta_{p}$ are the density and compressibility of particle, respectively, and $\rho_{0}$ and $\beta_{0}$ are the density and compressibility of the medium, respectively. Based on the previous equation, the magnitude of acoustic radiation force is a function of acoustic contrast factor. Higher values of $\phi$ determines larger force on the particle. In addition, acoustic contrast factor determines how the particle is moved in a standing wave. Positive values of $\phi$ affords nodal migration while negative values of $\phi$ affords anti-nodal migration (Figure 1.22). Sorting and separation based on acoustophoresis is possible using either the nodal/anti-nodal focusing or fractionation based on their differences in migration velocity due to their size difference. ${ }^{125}$ The size dependency of the 


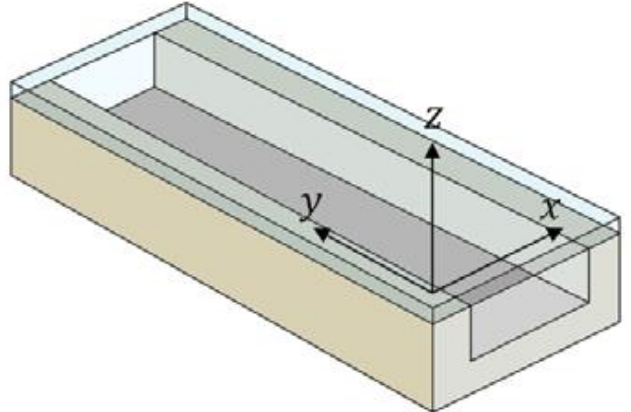

a)

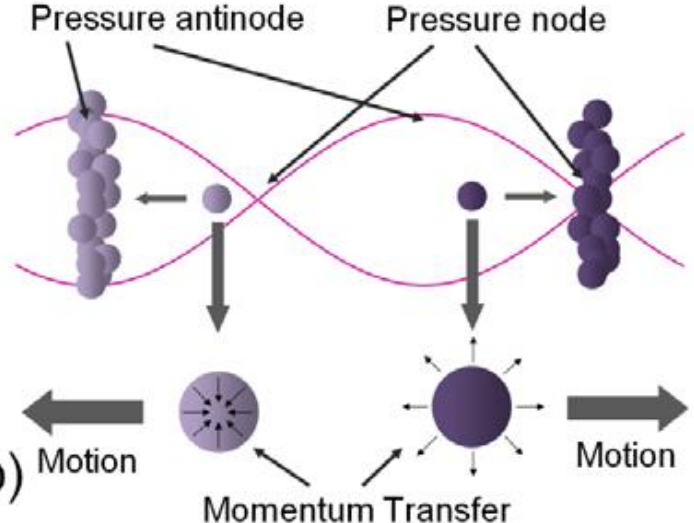

Momentum Transfer

Figure 1-22 Representing a) an acoustically actuated microfluidic device. The transversal direction of the channel and the flow direction in the channel are shown as $\mathbf{y}$ and $\mathbf{x}$, respectively. b) schematic of ultrasonic standing wave along with its pressure nodes and antinodes and the direction of migration for different particles in acoustic standing wave (Reprinted with permission from ${ }^{129}$ ).

migration velocity of particles is shown in the following equation. This equation results from the balance of acoustic radiation force and the drag force in the $\mathrm{x}$ direction:

$$
\sum F_{x}=F_{x}^{r a d}+F_{x}^{d r a g}=0 \Rightarrow v_{x}=\frac{2 k_{x} r^{2} E_{a c}}{3 \mu} \phi(\rho, \beta) \sin \left(2 k_{x} x\right)
$$

According to this equation, the migration velocity is a function of square of the particle radius. Therefore, a separation based on size of the particles in a standing acoustic wave is due to the differences in their velocity.

\subsubsection{Acoustic Particle Manipulation}

The application of an acoustic radiation field, as mentioned in section 1.2.3.4, and more specifically ultrasonic standing waves in a media containing suspended particles exert a force on these particles that could yield to their separation. ${ }^{124}$ The magnitude of this force is a function of the particle distance from the pressure node/antinode for nodal/antinodal focusing. ${ }^{125,130}$ For sorting and manipulation of particles in a microfluidic channel using acoustophoresis, the wavelength of the standing wave should be in the range of active sorting regions of the microfluidic channel, meaning that frequencies of few megahertz are required for a microfluidic channel with the size of an active force region of few hundred microns. Figure 1.23 shows schematic cross-sectional representation 
of particles in a microfluidic channel with the resonator placed at one side of the channel and the other side of the channel acts as reflector. When the actuator is off particles are randomly dispersed inside the channel (Figure 1.23 (a)). Figure 1.23 (b) shows the condition when the transducer is actuating at half-wavelength resonance mode and a standing wave with minimum and maximum points of pressure is formed. The pressure profile of the standing wave in this condition, which leads to the creation of a pressure node at the center and two antinodes at the walls, is shown with purple curves. The relative magnitude of the applied force is shown with blue curve. The direction of migration of particles (toward the pressure node) is indicated with blue arrows in the picture. As it was mentioned earlier, the migration direction depends on the sign of $\phi$ which is itself a function of density of the particle and medium. Based on the equation of $\phi$, it can be concluded that particles with densities higher than the density of the medium render a positive $\phi$ and will migrate toward the pressure node (Figure 1.23 (c)).
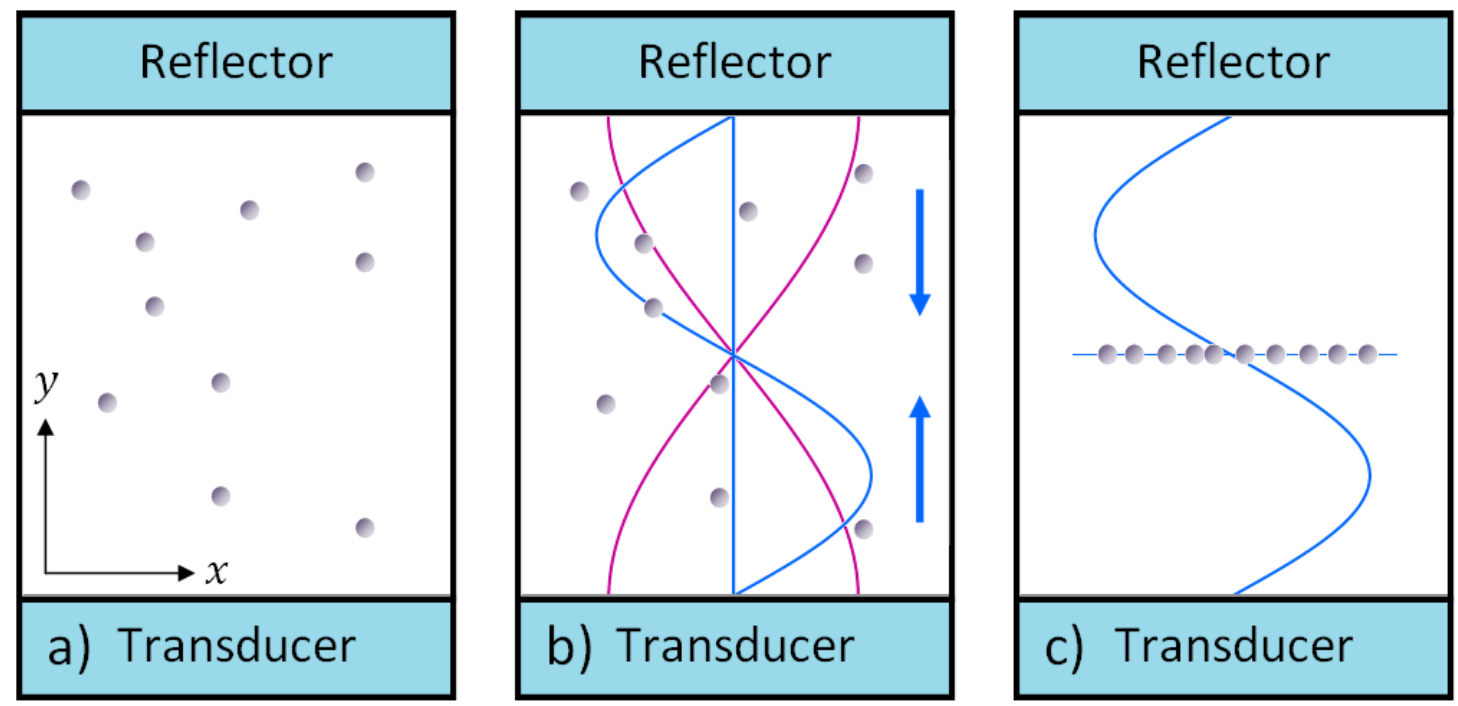

Figure 1-23 Illustration of particle movement in a standing wave acoustophoresis: (a) In the absence of acoustic radiation. (b) When acoustic radiation is activated at a half-wavelength resonance, resulting in the migration of particles toward the central pressure node which is shown with a purple line. The blue arrows show the direction of net force applied on the particles in such acoustic radiations. (c) the accumulation of the particles at the central node due to the acoustic radiation force. (Reproduced with permission from ${ }^{124}$ ). 
The acoustic radiation force has the ability to filter or concentrate particle based on their physical properties such as density and compressibility. ${ }^{124,131}$ This force can be used in a microfluidic channel to move the suspended particles toward the node or antinode locations and then the particles can be collected taking advantage of gravity and removing of the acoustic force. Figure 1.24 shows schematically the sedimentation of the particles using acoustic sedimentation technique. ${ }^{124}$

This method of concentration is useful in stationary and batch processing when the particles suspended in their carrier fluid is inserted in a one-time process. However, if the design of the microfluidic channel is modified such that an outlet is fabricated in the system, the continuous concentration and separation is possible. In this method particles suspended in a media are injected into the microfluidic channel. While passing through the region where the acoustic standing waves are presented, the migration and sorting happens. Figure 1.25 shows the design for such microfluidic device. There are three outlets fabricated in this device. The middle one is intended to collect focused particles and the carrier fluid is flowing out through the upper and lower outlets. ${ }^{132}$ In another application of acoustophoresis, the aim is to wash cells and particles from one fluid to another.
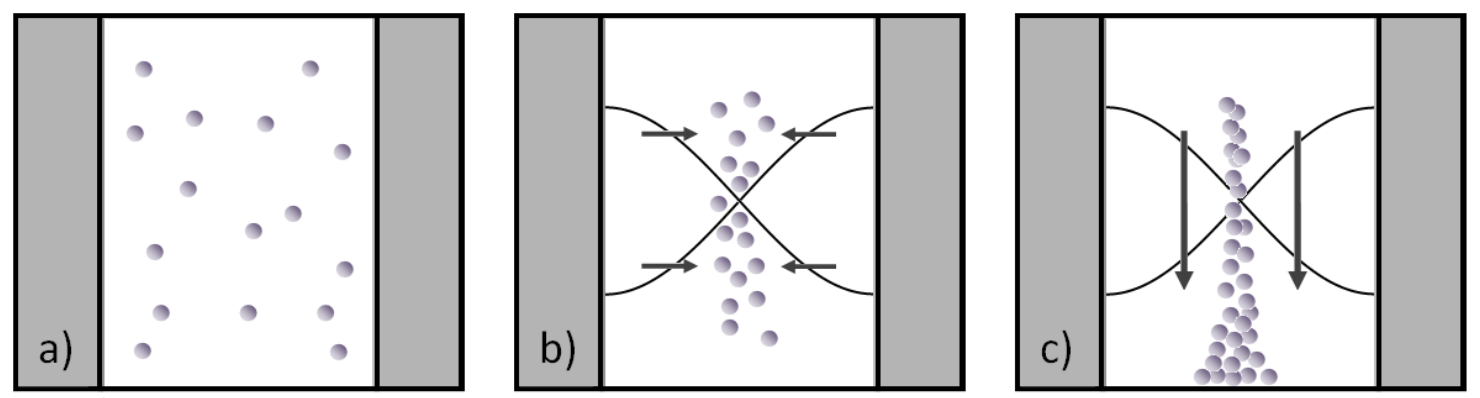

Figure 1-24 Showing the acoustic sedimentation technique: (a) Prior to the acoustic actuation particles are randomly dispersed in the channel. (b) Upon actuation particles start to accumulate at the center line of the channel which is the pressure node at half-wavelength resonance mode. (c) Accumulated particles start to sediment due to gravity. (Reproduced with permission from ${ }^{124}$ ). 


\section{Clear Fluid Out}

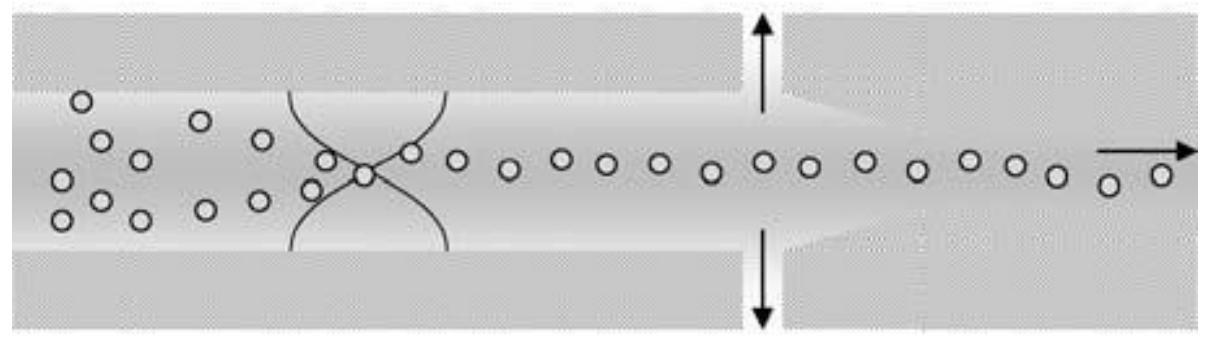

\section{Clear Fluid Out}

Figure 1-25 Schematic of a continuous microfluidic channel for the separation of particles from their carrier fluid. Particles are focused in the middle of the channel and are separated from the middle outlet and the medium is collected from the upper and lower outlets (Reproduced with permission from ${ }^{132}$ ).

This is possible as the fluids in the microfluidic channel flow with a laminar characteristic and are able to move throughout the channel without mixing. In such systems the device has to have two inlets, one of which for the insertion of particles with carrier fluid and the other one for the insertion of the target fluid. The acoustic radiation force is set in the channel such that the nodal region is placed in the target fluid, therefore, the particles migrate from the carrier fluid to the nodal region in the target fluid without mixing of the two fluids. Two outlets are designed at the end of the channel to collect each fluid separately (Figure 1.26). ${ }^{133}$

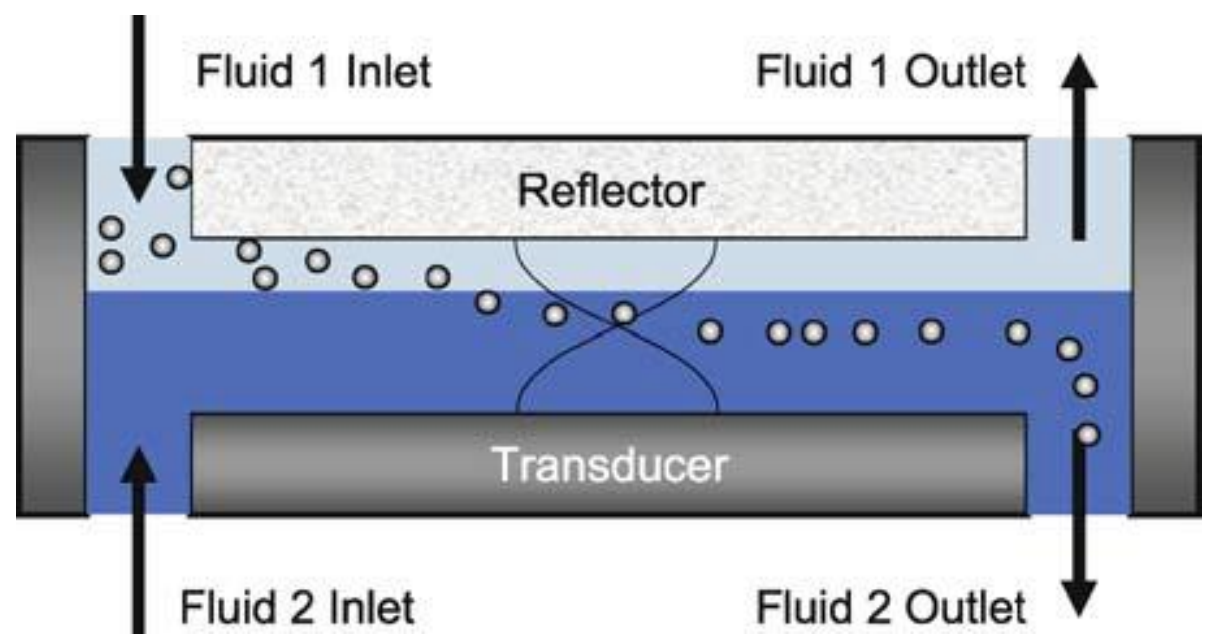

Figure 1-26 Washing particles from one medium to another using acoustic radiation under laminar flow. When the acoustic radiation is adjusted to produce the pressure node in the second medium, particles tend to migrate from the first carrier medium to the second one (Reproduced with permission from ${ }^{133}$ ). 
The acoustic standing waves in the above mentioned acoustofluidic devices were designed such that the nodal region lies in the center of the microfluidic channel and there were two antinodes at the walls. These devices work in the half-wavelength resonance mode. However, in some applications, it is preferred to have other modes of actuation. For example, acoustophoresis can be used to improve the sensitivity of biosensors in biomedical assays. ${ }^{124,134}$ In these types of application, the resonance would be in a quarter-wavelength mode in which a nodal region is constructed at the reflector wall where the biosensor is fabricated and the antinodal region is formed in the opposite wall of the channel that is the resonator surface (Figure 1.27). The number of the particles that migrate to the sensor at the reflector wall is increased due to the acoustic radiation force. It was shown that the concentration of Bacillus subtilis var. niger spores that are sensed using acoustic radiation method is increased 70 times, which shows the capability of this method to enhance the sensitivity of biosensors. ${ }^{135}$ Another frequently used application of acoustic force in the microfluidic devices is to separate and sort particles of the same type based on their size. As it was explained in the acoustic radiation force equation (section 1.3.3), the force exerted on a particle is a function of $r^{3}$ with $r$ being the radius of the particle.

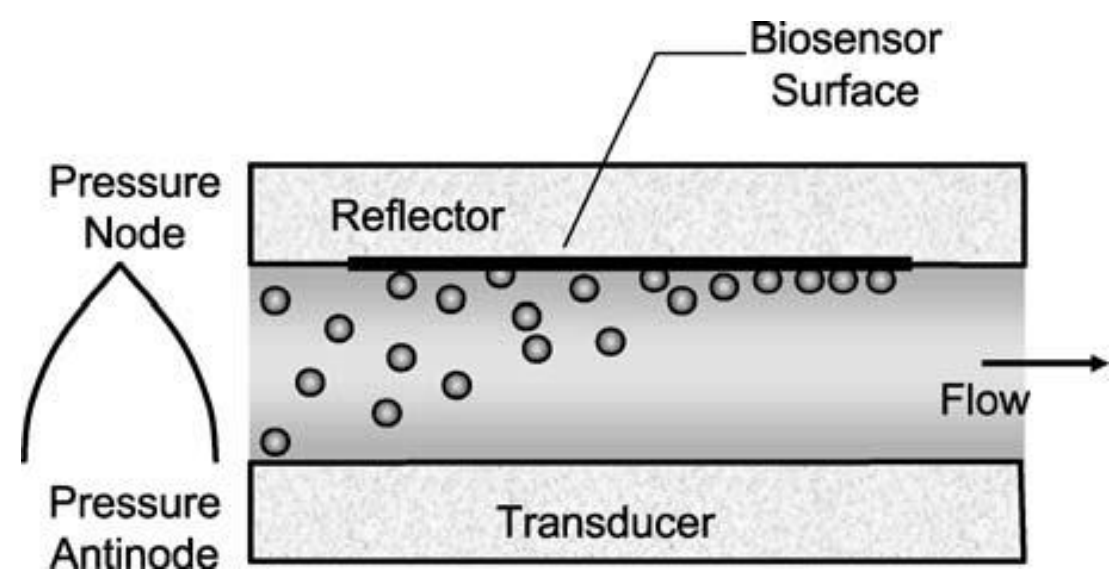

Figure 1-27 Acoustic radiation resulted in an improvement in capturing bacteria by the biosensor. The sanding wave in this case is working in a quarter-wavelength resonance mode so that the pressure node is formed on the biosensor side wall, therefore increase the capture of bacteria on the sensor (Reproduced with permission from ${ }^{135}$ ). 


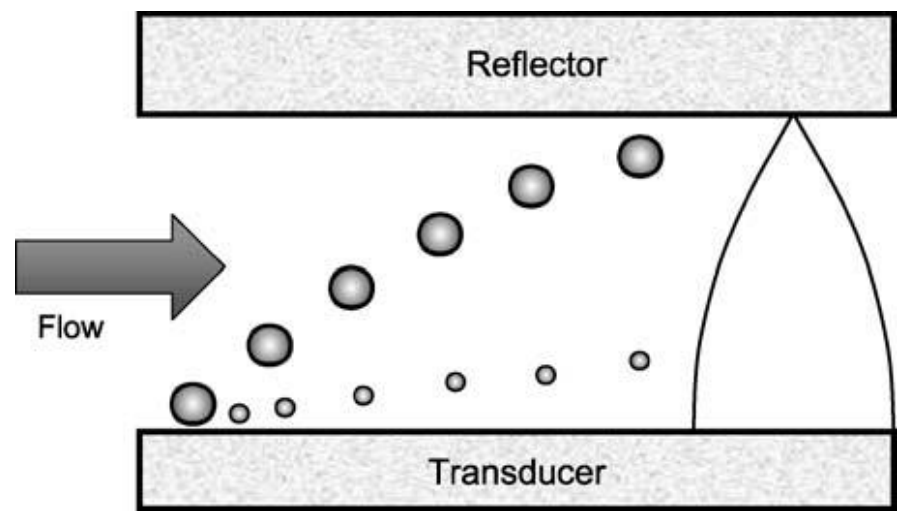

Figure 1-28 Acoustofluidic separation of particle based on their size using a quarter-wavelength resonance. The size of the particle determines the magnitude of the force exerted on them. Based on the difference in size, particles are focused in different locations at the standing wave (Reproduced with permission from ${ }^{124}$ ).

Also the migration velocity is a function of $\mathrm{r}^{2}$. Using the differences in acoustic force and migration velocity yielded by differences in the size of particles, in a laminar flow condition, a separation system could be developed. It should be noted that the residence time should be selected carefully to avoid the accumulation of all particle sizes in the node. Figure 1.28 shows an example of the microfluidic device for size-based separations. In this device also a quarter wavelength resonance mode is applied to the channel and particles are differentiated due to their differences in the acoustic radiation force.

\subsubsection{Acoustophoresis of Biological Particles}

Acoustophoresis as a method of separation of particles has been investigated in many studies. ${ }^{136}$ The differentiation via acoustophoresis is based on the physical property differences in the particles. In contrast to other method of separations, acoustophoresis has the least effect on the particle which makes it a reliable candidate for the separation of biological samples. ${ }^{137}$ It also offers a relatively rapid and easy to employ screening technique. ${ }^{138}$ There has been several studies that consider acoustophoresis as a practical separation technique for cell separation. ${ }^{139,140}$ However, similar to other methods of separation, acoustophoresis also encounters some limitations with regards to further improve the separation efficiency. One of the most important limitations is the 
fact that different cell types have been shown to have similar acoustophoretic behavior. Meaning that cell of the same size tend to migrate toward the high pressure nodal region regardless of their type. ${ }^{141}$ It is assumed that the differences in their compressibility is not high enough to yield a difference in their acoustic radiation force. This section focus on some of the recent applications of acoustically actuated microfluidic systems for manipulation and sorting of biological particles like cells.

As it was mentioned earlier (section 1.3.5), acoustophoresis can be used to wash particles from one fluid to another. This feature is highly important for many bioanalytical studies. Hawkes et al. used an acoustofluidic technique, similar to what has been presented in Figure 1.26, for transferring yeast cells from one fluid to another. The laminar flow along with a continuous FFF were used to insert two different media into the channel without mixing. Similar to Figure 1.26, a half-wavelength resonance standing wave was created inside the channel which forms a nodal region at the center of the channel. One of the two inlets is to inject yeast cells suspended in a media and the other inlet is used for the insertion of the second media. Here also on the actuation cells migrate to the center of the channel which is formed in the second media and are carried out through the designed outlet. ${ }^{142}$ The same concept was used by Tenje et al. ${ }^{143}$ where the plasma proteins and other lowmolecular weight compounds were washed away from red blood cells (RBCs) and RBCs are resuspended in a buffer solution (Figure 1.29). Using this method higher concentration efficiencies are achieved that was not feasible otherwise. Preparation of protein-free RBC is of high importance for blood transfusion to whom suffering from Immunoglobin A (IgA) deficiency. Immunoglobin A (IgA) deficiency is a common immunodeficiency disease in which the patient has an undetectable levels of IgA in the blood. In a study by Antfolk et al., a microfluidic chip was developed for the separation of CTCs from white blood cells (WBCs). 


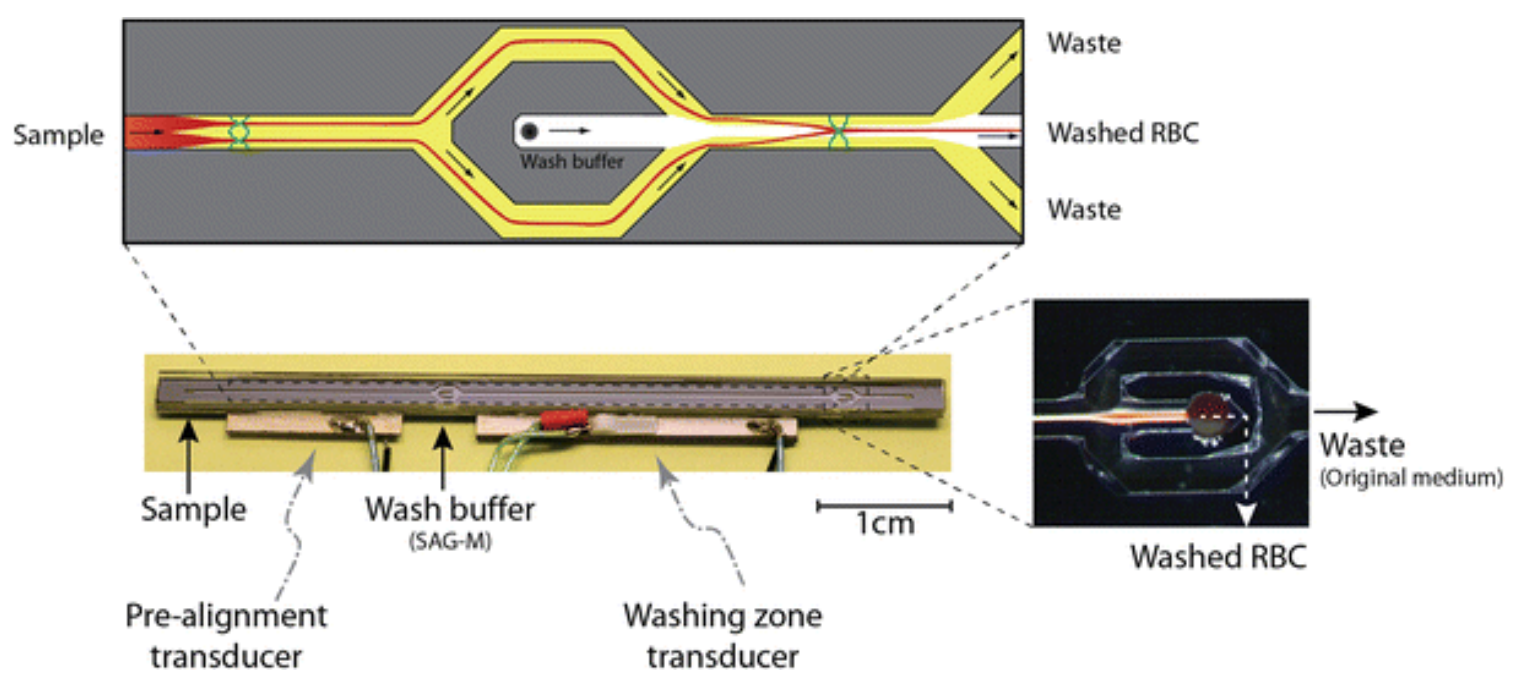

Figure 1-29 Removing proteins from red blood cells (RBC) using acoustophoresis. Protein-free RBCs are collected from the center outlet (picture on the right shows the outlet and collected RBCs). (Reprinted with permission from ${ }^{143}$ )

This device had a single inlet and consequently single laminar flow was created inside the channel and two piezoelectric transducer were used for alignment and separation of cells. The high rate of recovery achieved by this method could further be increased using higher acoustic radiation intensity. ${ }^{144}$ The ability of acoustophoretic to distinguish between CTCs from blood cells based on their intrinsic properties offers a promising method of separation for bioanalytical studies. Other methods of separation were limited due to losing of a lot of characteristics of epithelial cells during metastasis, which is known as epithelial-mesenchymal transition (EMT). ${ }^{144,145}$

In another study by Li et al. ${ }^{146}$, CTCs were successfully separated from peripheral blood of the patients with breast cancer cells. In this study, tilted-angle standing surface acoustic waves (taSSAW) were constructed in the acoustophoretic device to achieve a successful separation of: 1) cultured human breast cancer (MCF-7) and cervical cancer (HeLa) cells from WBCs, and 2) rare breast CTCs from WBCs while the viability was maintained (Figure 1.30).

taSSAW is a recently developed technique in which higher efficiency and sensitivity was obtained compared to the conventional methods using a numerically optimized configuration of tilted standing wave. 


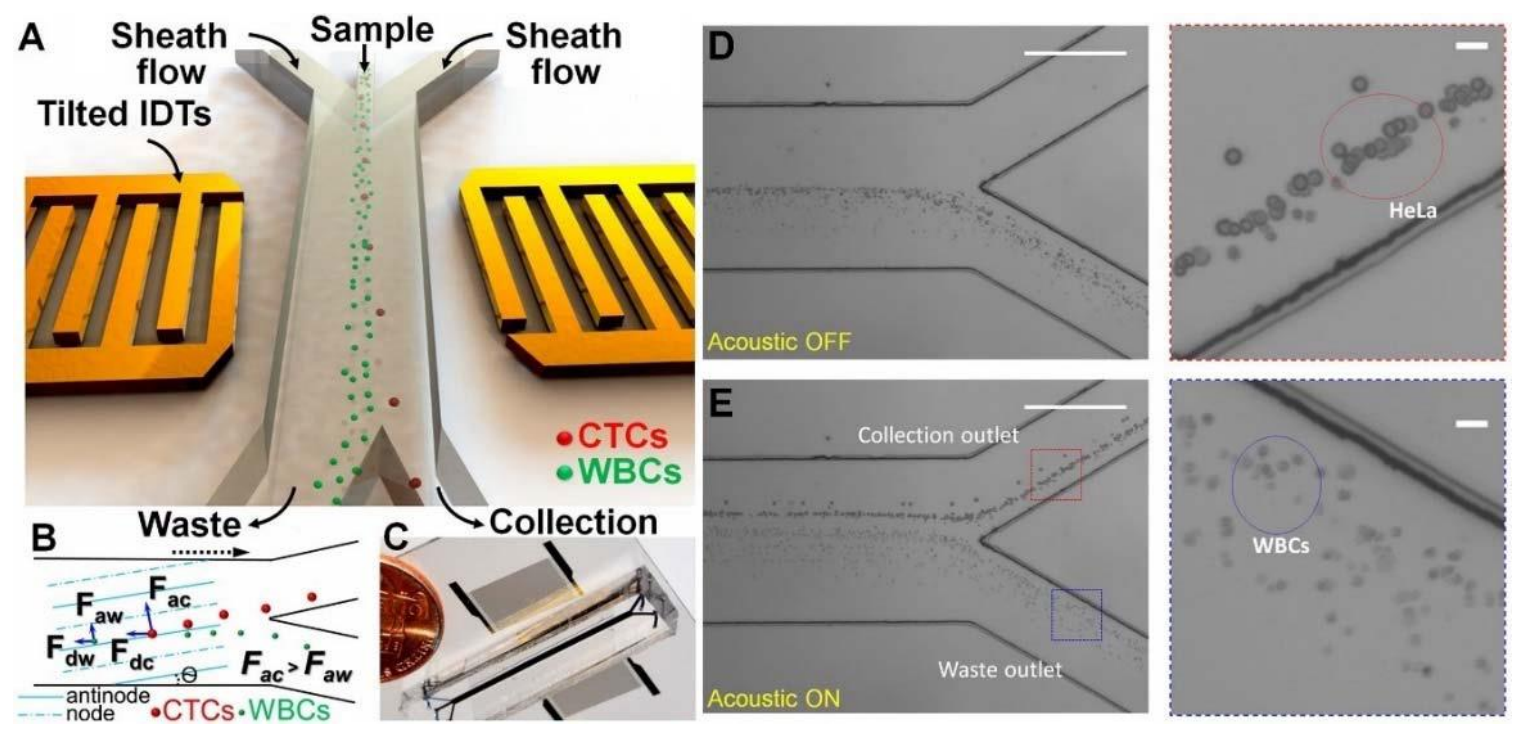

Figure 1-30 a) Showing the schematic of taSSAW device. b) The node and antinode in taSSAW are at an angle with regards to the flow direction. As a result, particles with larger sizes such as CTCs undergo greater acoustic force in comparison with WBCs, they therefore migrate to farther distances perpendicular to the flow direction. c) Image of an actual taSSAW microfluidic system. d) Showing a mixture of HeLa cells and WBCs inserted in the channel with no acoustic actuation. All the particles are collected from the lower outlet and no separation occurs. e) The same mixture upon acoustic actuation are separated based on their size. HeLa cells are larger, so they experience greater force and migrate toward the upper outlet, while WBCs are collected from the lower outlet. (Reprinted with permission from ${ }^{146}$ ).

The reported efficiency for the size-based separation of polystyrene microbeads was as high as 99\%. Other than high efficiency for size based separation, this method is shown to be capable of separating based on the compressibility difference of particles and cells of the same size and density. ${ }^{147}$ It was mentioned earlier that the separation of same size cells with acoustophoresis faces some challenges as the difference in their densities are not large enough to make a detectable contrast in their acoustic contrast factor and acoustic radiation force. However, Wyatt Shields et al. proposed a "particle-based" method to overcome this challenge. In this method, cells were connected to elastomeric particles which renders negative acoustic contrast factor. Therefore, they would migrate toward the antinodal regions while those cells that are not bound to those particles (with positive acoustic contrast factor) would migrate toward the nodal regions of the channel. Figure 1.31 shows the antinodal migration of cells as they bound to elastomeric particles. ${ }^{148}$ 

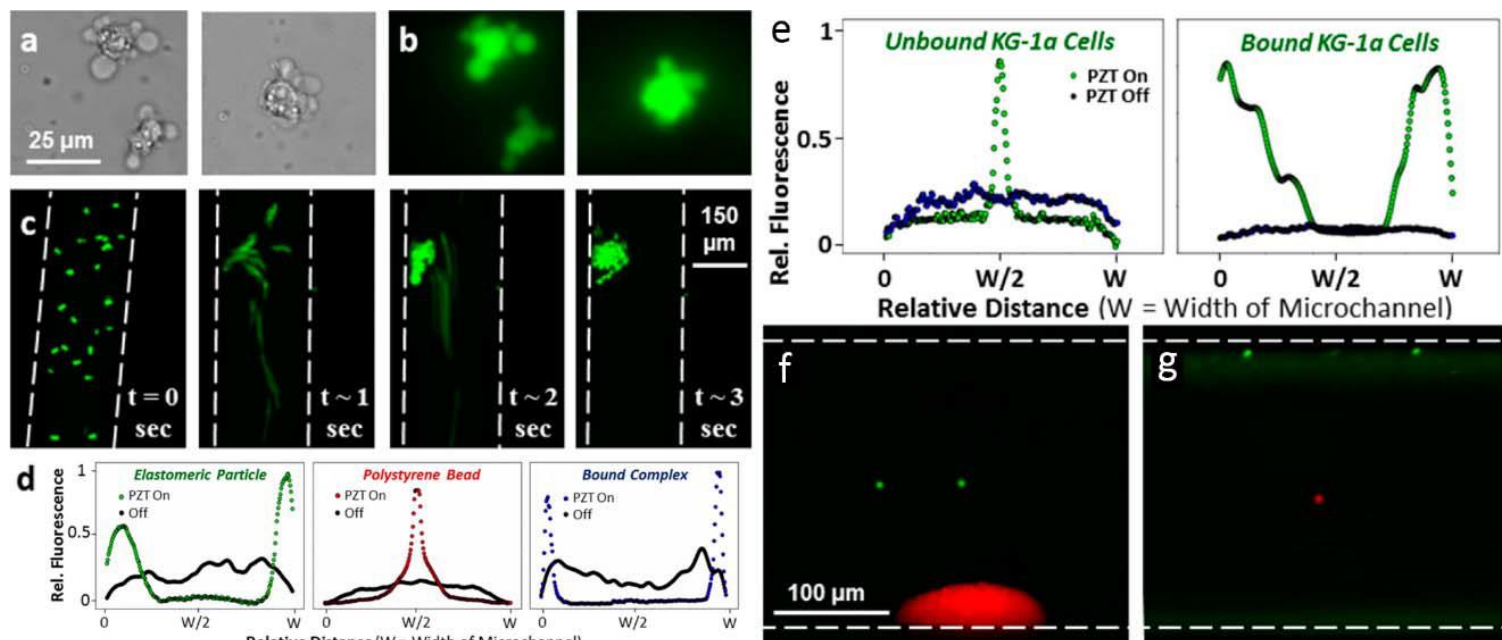

Relative Distance $(\mathrm{W}=\mathrm{Width}$ of Microchannel)
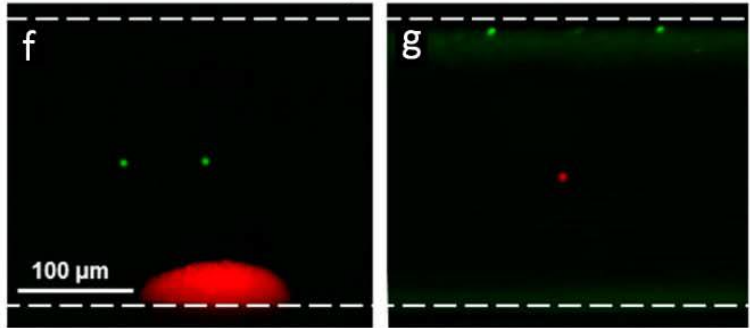

Figure 1-31 Showing the binding of elastomeric particles to cells for the separation of cells: a) Brightfield, and b) Fluorescent micrograph of SA-adsorbed particles that are attached to KG-1a cell (labeled with green dye). c) Showing the migration of cells with their attached elastomeric particles as the acoustic actuation turns on. d) From left to right: intensity distribution of SA-adsorbed particles, biotinylated polystyrene microbeads and complexes of particles along the channel with the width of W. e) The intensity profiles for the separation of particle-bounded cells from particle-free cells. f) fluorescence micrograph of KG-1a cells (green labeled) focused at node and elastomeric particles tagged with Nile red dye focused at the antinode. And g) the separation of SA-adsorbed elastomeric particles attached to the anti-CD34-biotin cells (green labeled) from the cells without anti-CD34-biotin (red labeled). (Reprinted with permission from ${ }^{148}$ )

\subsection{Statement of the Problem}

Cellular Stiffness and compressibility as the biomechanical properties of cells have been under investigation in bioanalysis research as biomarkers for diseases. For instance, changes in the level of stiffness in cells has been related to an underlying pathology within the cells. Likewise, various other conditions, including but not limited to coronary heart diseases, parasitic infections, red blood cell anemia, and neurodegenerative diseases have been correlated to changes in the cellular stiffness. Separation and analysis of the cells undergone these conditions or the extracellular vesicles produced by the affected cells provides valuable information regarding the pathology of the disease and is of great diagnostic and therapeutic values.

Acoustophoresis is a label-free, gentle, and high-throughput separation technique that is increasingly being used in the separation of cells and other bioparticles. This method, apart from 
its intrinsic capability in making differentiation based on compressibility, has been shown to be unsuccessful in separating cells and bioparticles with varying mechanical properties. Therefore, acoustophoresis has been mainly limited for the unaltered separation of cells based on their size contrast. The main observation arising from studies involving cell separation under acoustophoresis was that different cell types seemed to behave similarly, i.e. migration to the high-pressure zone(s) or node(s) of the ultrasonic standing wave. However, depending on the cell type and its pathological condition, cellular rigidity changes extremely among cells, varying as much as 10 folds in stiffness in some instances. To our knowledge, no previous works were able to harness this vast changes in stiffness for same size and shape cells to achieve separation in acoustophoresis.

Previously in our group, we investigated a novel technique which was a departure from conventional acoustofluidic technologies and in that for the first time thermally-induced changes in the physical properties of membrane-bound bioparticles was used to yield separations that are otherwise impossible using conventional methods. In this method, to differentiate between vesicles of same size but different compositions, the sensitive response of vesicles' compressibility due to temperature changes was harnessed. Because of the thermotropic transitions in their phases, vesicles are expected to exhibit a positive to negative $\phi$ change at a unique temperature based on the lipid composition of their membrane. Following this transition, caused by ordered to disordered phase transition upon heating, the stiffness or compressibility of the membrane of vesicles changes (more compressible or less stiff). Therefore, a separation was achieved based on differences in the distinct transition temperatures of two distinct vesicle compositions in the microfluidic device when operating at a separation temperature where $T_{\text {separation }}$ is in between the $T_{\text {transition }}$ of the vesicles.

Other than lipids makeup, protein contents also affect the characteristics of the membrane and therefore change the transition temperature of the vesicles. The initiative undertaken in this study will be departure from what has already been developed in our lab and move forward with evaluating the capabilities of thermally-assisted acoustophoresis method for the separation of same 
size vesicles which differ to one another in protein content. Also for the first time use this technique for the separation of cells based on their thermally-induced changes in their physical properties. Figure 1.32 (left) depicts the expected separation of two types of vesicles, each experiencing a different $\phi$ sign at $T_{\text {transition,ves1 }}<T_{\text {separation }}<T_{\text {transition,ves2 }}$ and (right) represents the building blocks of a liposomal vesicle containing transmembrane proteins. It is anticipated that as long as a safe temperature range is employed $\left(1-43^{\circ} \mathrm{C}\right)$, no permanent change to the genetic or proteomic properties of the biological vesicles will occur.
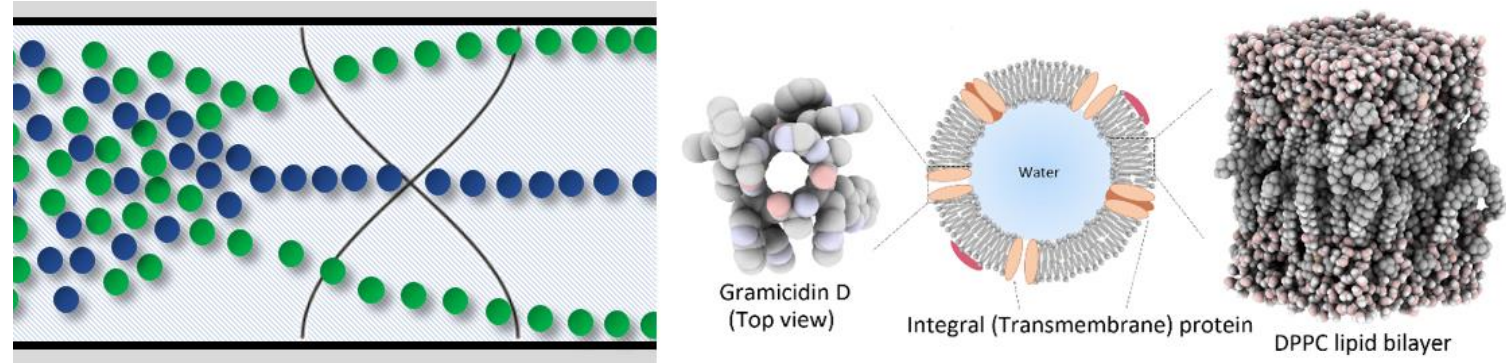

Figure 1-32 Schematic representation of a separation event (left), and building blocks of a lipid vesicle containing a transmembrane protein. 
2 Materials and Methods

\subsection{Thermo-Acoustophoretic Setup}

The thermo-acoustophoretic setup consisted of microfluidic channel in which the separation takes place and a piezoelectric transducer which is placed underneath the chip to produce acoustic excitation. A function generator and a power amplifier were used to generate and amplify an AC signal of sinusoidal form. During the thermo-acoustophoretic experiments, the chip temperature was controlled using a Peltier element. The flow at the inlet and outlets were controlled by syringe pumps (New Era) holding 1-mL syringes (BD Luer-Lok ${ }^{\mathrm{TM}}$ ) connected to luer ends-equipped PVC tubing (Cole-Parmer). The following sections describe each particular component in more details.

\subsubsection{Microfluidic Device}

The microfluidic device used in this study was designed to produce a transversal resonance meaning that the standing waves are formed by the reflections from the walls of the channel. The mode of the resonance in transversal design is perpendicular to the actuation direction. For the microfluidic channel to maintain a proper acoustic resonance, it should be made of a material with low acoustic losses and a high difference in acoustic impedance with respect to the one of the fluid. ${ }^{149}$ A schematic of a transversal resonator is shown in Figure 2.1. ${ }^{149}$

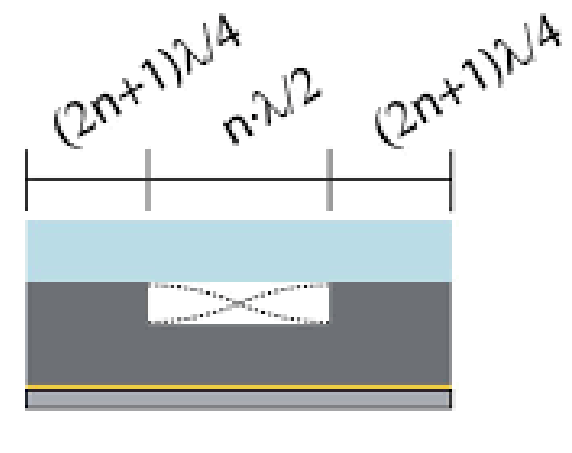

\section{Transversal resonator}

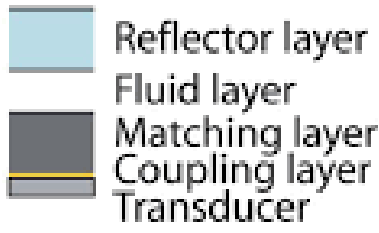

Figure 2-1 Schematic illustration of a microfluidic channel resonating in transversal direction with optimum dimensions. (Reprinted with permission from ${ }^{149}$ ) 
The suitable dimension for a half-wavelength transversal resonance (width of the channel $=n \frac{\lambda}{2}$ )

is where the bulk material surrounding the channel has the width equal to $\frac{3 \lambda}{4}$. In this study the microfluidic channels were made of 4-inch <100> silicon wafer with thickness of 500-550 $\mu \mathrm{m}$ purchased from WRS Materials. All wafers had a $1.000 \pm 0.050 \mu \mathrm{m}$ thick low-stress silicon nitride $\left(\mathrm{Si}_{3} \mathrm{~N}_{4}\right)$ coating on both sides. This coating layer, that was acting as a mask during the etching process with potassium hydroxide $(\mathrm{KOH})$ to remove the bulk underlying silicon and make the chamber for the channel, was eventually stripped off from the surface using another wet etch process. The fabrication process of microfluidic channel is described in more details in section 2.2.

\subsubsection{Device Cover Lid}

The microfluidic channel should be covered with a material that has a comparable/similar acoustic impedance to the one of substrate in order to maximize the energy density of the acoustic radiation and minimize the acoustic loss due to the reflection from the lid. ${ }^{149}$ Borosilicate glass is one of the most commonly used materials as it can be attached to the silicon channel via anodic bonding. This strong bonding leads to the uniforms performance of the entire setup as a single structure. The transparent glass cover also enables the visualization throughout the experiments. For the cover lid of microfluidic devices in this study, 4-inch BOROFLOAT ${ }^{\circledR} 33$ borosilicate glass double-side polished wafers (Präzisions Glas \& Optik GmbH) with the thickness of $0.700 \pm 0.025 \mathrm{~mm}$ were used. The thermal expansion coefficient of the BOROFLOAT® borosilicate glass wafer is almost the same as that of silicon. Acoustic impedance of these two material is very similar. Both of thses materials are resistant to high temperatures which makes them suitable for anodic bonding.

\subsubsection{Piezoelectric Transducer}

The acoustic transducer used here is an intermetallic inorganic compound, lead zirconate titanate (PZT), purchased from APC International and has remarkable piezoelectric properties. Table 2-1 
summarize the specifications of APC 850 and 851 piezoelectric transducers that were used in this study.

Table 2-1 Characterization of APC 850 and 851 piezoelectric transducers

\begin{tabular}{|c|c|c|c|c|c|c|}
\hline $\mathrm{Pb}[2$ & $\begin{array}{l}\text { terial: } \\
\text { Ti1-x]O3, } \\
\leq \mathrm{x} \leq 1\end{array}$ & Shape & Electrode Pattern & Thickness & Width & Diameter/Length \\
\hline $\begin{array}{l}\text { Type } \\
850\end{array}$ & $\begin{array}{l}\text { Structure } \\
\text { perovskite }\end{array}$ & & & & & \\
\hline $\begin{array}{l}050 \\
850\end{array}$ & perovskite & $\begin{array}{l}\text { Disk } \\
\text { Disk }\end{array}$ & Wrap around & $2.20 \mathrm{~mm}$ & N/A & $9.50 \mathrm{~mm}$ \\
\hline 851 & perovskite & Plate & Solid & $0.70 \mathrm{~mm}$ & $13.00 \mathrm{~mm}$ & $70.00 \mathrm{~mm}$ \\
\hline
\end{tabular}

\subsubsection{Temperature Control System}

Since the working frequency of this work may cause about 3 to $4{ }^{\circ} \mathrm{C}$ temperature increase, a Peltier element (Farnell) was used to adjust the temperature of microfluidic device. This Peltier element was connected to a DC power supply (Model 72-2010, TENMA). For a uniform temperature distribution, Peltier element was placed in between two aluminum plates. Temperature measurement was performed using a flat platinum resistance thermometer (PT100, Thermometrics) with the repeatability of $\pm 0.1^{\circ} \mathrm{C}$ which was placed on the backside of the device, close to the main channel. An IR thermometer was used on top of the channel to proofread the temperature measurements.

\subsection{Device Microfabrication}

In this section the microfabrication process of the thermos-acoustophoretic device is described. This process consists of a series of standard photolithography procedures to create a microfluidic channel into the silicon wafer and cover it with a glass wafer using a suitable bonding technique.

\subsubsection{Mask Design and Pattern Generation}

The first step in every photolithography procedure is to design the layout of the device. Here, the channel layout was first designed by the CAD software Layout Editor (Juspertor Gmbh) and then 
transferred onto a photomask via high-precision laser mask writer ( $\mu \mathrm{PG} 101$ Heidelberg Instruments, Mikrotechnik GmbH). The photomask used here was a soda lime glass, pre-coated with a $1000 \mu \mathrm{m}$ thick chromium and $5300 \AA$ thick positive-tone photoresist (AZ1518). The parameters of mask writer for patterning were $100 \%$ exposure rate with $18 \mathrm{~mW}$ of power. After writing the pattern on the mask, the exposed photoresist on the mask was developed in a developer solution. For AZ1518 positive photoresist, the developer was AZ 400K (AZ Electronic Materials) diluted with water (4:1) for $70 \mathrm{~s}$. The chromium layer of those parts of mask surface which were not covered with photoresist was then removed through a wet etch process by soaking the wafer in a bath of a fully concentrated etchant (Chromium Etchant 1020, Transene Company, Inc.) for 1 minute. An oxygen plasma treatment was then performed for 660 seconds to further remove the remaining photoresist. The parameters used for fully stripping the photoresist via oxygen plasma was $400 \mathrm{mTorr}$ pressure of $\mathrm{O}_{2}, 100$ watts of power and $60 \mathrm{sccm}$ flow rate. The pattern developed on the mask was then transferred to the silicon wafer by UV exposure.

Before starting to transfer the pattern on the wafer, the wafer was thoroughly cleaned using a spin cleaning process with acetone and isopropyl alcohol (IPA). In the next step, bis(trimethylsilyl)amine (also known as hexamethyldisilazane, or HMDS) which acts as the adhesion promoter was applied through the spinning process of $3000 \mathrm{rpm}$ for 30 seconds on the wafer. Then, the wafer was spin coated with AZ 4620 (MicroChemicals GmbH) in two back-toback steps: 1) 5 seconds at $100 \mathrm{rpm} / \mathrm{s}$ up to $500 \mathrm{rpm}$ and 2) 30 seconds at $300 \mathrm{rpm} / \mathrm{s}$ up to 2500 $\mathrm{rpm}$. The coated wafers were then undergone a soft bake process at $110^{\circ} \mathrm{C}$ for $2 \mathrm{~min}$.

To precisely transfer the pattern from mask to the wafer, a mask aligner (Model $800 \mathrm{MBA}, \mathrm{OAI}$ ) was used in contact-mode with the recommended exposure energy of $400 \mathrm{~mJ} / \mathrm{cm}^{2}$. The developer used to develop the pattern on the wafers was also the dilution of 1:4 (volume ratio) of developing solution (AZ® 400K, AZ Electronic Materials) in DI water. Optical microscopy (Unitron Versamet 
Optical Microscope) was employed to examine the features of the design on the developed wafers. Then, a descum plasma treatment, with oxygen as the process gas, was applied to completely remove the resist from the channels' trench. The parameters of reactive ion etcher (CS-1701, MARCH) for the descum plasma treatment was 400 watts of power and 100mTorr pressure of $\mathrm{O}_{2}$ entering the chamber with $60 \mathrm{sccm}$ of flow rate for 1 minute. The silicon nitride layer on the cleaned wafers were then removed using the same instrument this time with tetrafluoromethane (CF4) as the process gas. After removing the nitride layer, the photoresist stripping were performed on the wafers using a Remover PG solution (MicroChem) at $60{ }^{\circ} \mathrm{C}$ for 10 minutes. This process was followed by a thorough rinse with IPA, then water and dry.

\subsubsection{Etching}

Before starting to etch the wafers, all the patterned features were carefully examined via optical microscopy. The anisotropic wet etching process was then employed to remove the underlying silicon material on the wafers using a preferential silicon etchant (PSE-200) (Transene Company, Inc.). The wet etching was continued until the desired depth of channel $(90 \mu \mathrm{m})$ was gained. A profilometer (Alpha-Step 200, KLATencor) was used to measure the depth of the channel. The next step was to remove the remaining nitride from the surface of the wafers. Before doing so, wafers should be rinsed with ethanol and DI water and then dried. The wet etching process for removing the nitride layer was performed using an aluminum etchant (Transene Company, Inc.) at $180{ }^{\circ} \mathrm{C}$ for 210 minutes. Piranha solution (3 parts of sulfuric acid $\left(\mathrm{H}_{2} \mathrm{SO}_{4}\right)$ and 1 part of $30 \%$ hydrogen peroxide $\left.\left(\mathrm{H}_{2} \mathrm{O}_{2}\right)\right)$ was used to clean the wafers after nitride removal. The cleaning process was followed by rinsing with ethanol and DI water to remove any debris left on the wafers. The wafers were then again examined with optical microscopy. 


\subsubsection{Bonding of Glass Cover Lid}

Based on the design of the channel, inlets and outlets were bored onto the BOROFLOAT ${ }^{\circledR}$ glass wafers using a manual drill (220-01 WorkStation $^{\mathrm{TM},}$ Drememl). Prior to bonding the glass cover to the silicon channel, both parts were cleaned in piranha solution (sulfuric acid $\left(\mathrm{H}_{2} \mathrm{SO}_{4}\right)$ : $30 \%$ hydrogen peroxide $\left(\mathrm{H}_{2} \mathrm{O}_{2}\right)$; 3:1 and rinsed in acetone and DI water followed by drying. The anodic bonding was used to bond glass covers to the silicon substrate. For doing so, silicon wafers were heated up to $500{ }^{\circ} \mathrm{C}$ and then were anodically bonded for 15 minutes using a power supply (Model 247, Keithley Instruments Inc.) to produce $500 \mathrm{~V}$ potential difference. In anodic bonding, the coefficient of thermal expansion (CTE) of the bonded materials should be close to each other as this method does not allow differences in CTEs of two bonded materials. The CTE of BOROFLOAT® 33 borosilicate glass wafers was $3.25 \times 10^{-6} \mathrm{~K}^{-1}$, which was similar to the one of silicone wafer $\left(2.56 \times 10^{-6} \mathrm{~K}^{-1}\right)$ in the temperature range of $20-300{ }^{\circ} \mathrm{C}$. Figure 2.2 shows a microfluidic device fabricated with the above mentioned procedure and Figure 2.3 shows the process flow of device fabrication.

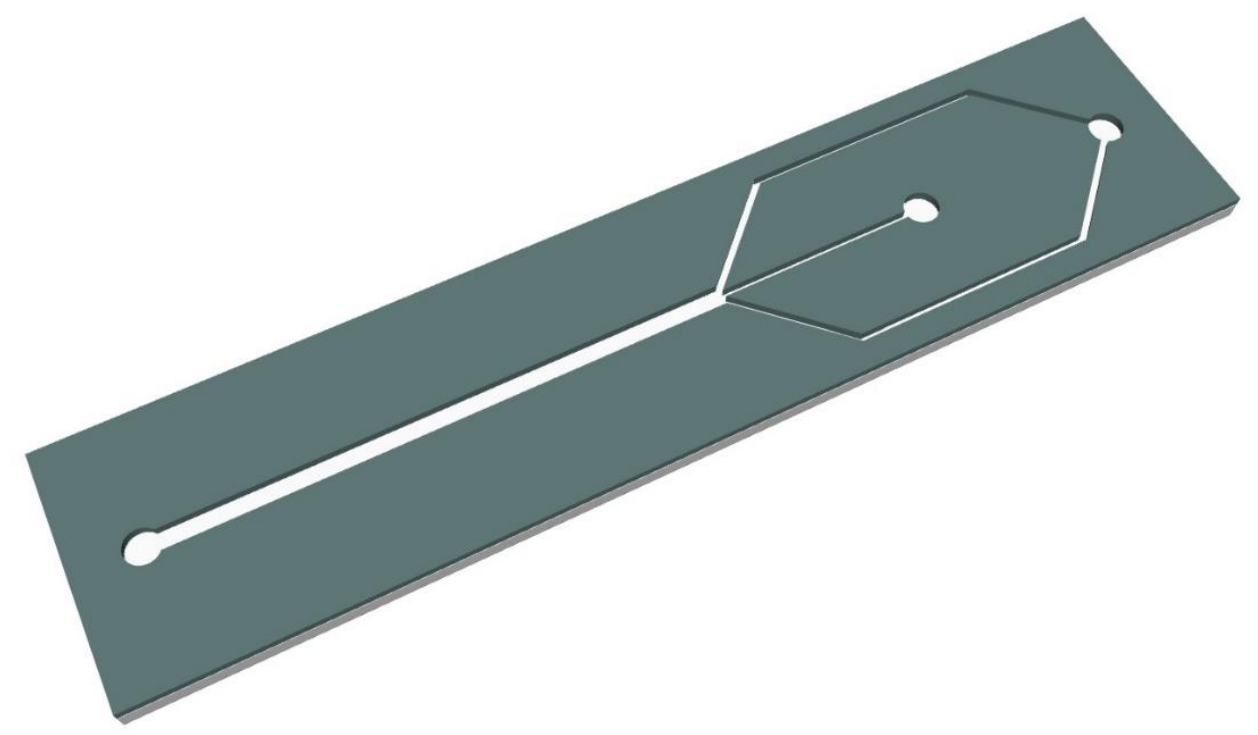

Figure 2-2 Image of the microfluidic separation device designed and fabricated for this study using standard photolithography procedure. 
The wafer used as the substrate was 4-inch $\langle 100\rangle$ prime silicon (WRS Materials), pre-deposited with a $1-\mu \mathrm{m}$ layer of low-stress silicon nitride $\left(\mathrm{Si}_{3} \mathrm{~N}_{4}\right)$.

The front side was spin coated by photoresist AZ 4620 (MicroChemicals), and then baked at $110{ }^{\circ} \mathrm{C}$ for $2 \mathrm{~min}$.

Using a contact-mode aligner (Model $800 \mathrm{MBA}, \mathrm{OAI}$ ), the wafer was exposed at the exposure energy of $400 \mathrm{~mJ} / \mathrm{cm}^{2}$.

Wafer was developed in $A Z^{\circledR} 400 \mathrm{~K}$ (AZ Electronic) prediluted with DI water at a volume ratio of 1:4.

The developed wafer was processed with tetrafluoromethane $\left(\mathrm{CF}_{4}\right)$ plasma to remove the nitride layer.

The remaining photoresist was stripped using a Remover PG solution (MicroChem) at $60{ }^{\circ} \mathrm{C}$ for 10 minutes.

The underlying silicon was removed by an anisotropic wet etching using a preferential silicon etchant (PSE-200) (Transene).

The remaining nitride was removed by wet etching using an aluminum etchant (Transene) at $180{ }^{\circ} \mathrm{C}$ for 210 minutes.

Next, 4-inch Pyrex ${ }^{\circledR}$ wafers (Praezisions Glas \& Optik) were anodically bonded to the heated-up substrates $\left(500{ }^{\circ} \mathrm{C}\right)$ using $500 \mathrm{~V}$ of potential difference provided by a power supply (Model 247, Keithley) for 15 minutes.

Assembly of the thermo-acoustofluidic device showing the chip layout, transducers, tubing and temperature sensor. The silicon/glass plates were clamped together via anodic bonding. In the close-up the position of the ultrasonic transducer is shown compared to the main acoustophoretic region in the channel.

Figure 2-3 Process flow of the fabrication of microfluidic device showing the photolithography process of the front side of silicon wafer after cleaning with acetone, IPA and HMDS. The process flow consisted of photoresist spin coating, followed by a soft bake, exposure and development in the proper developer solution. The developed pattern was then etched with RIE to remove nitride layer in the patterned area and produce a hard mask. Then, the exposed silicone was etched through with a wet chemistry etching process in $\mathrm{KOH}$. After etching the microfluidic channel, the remaining nitride layer was removed using an aluminum etchant and followed by cleaning, drying and anodic bonding of glass cover.
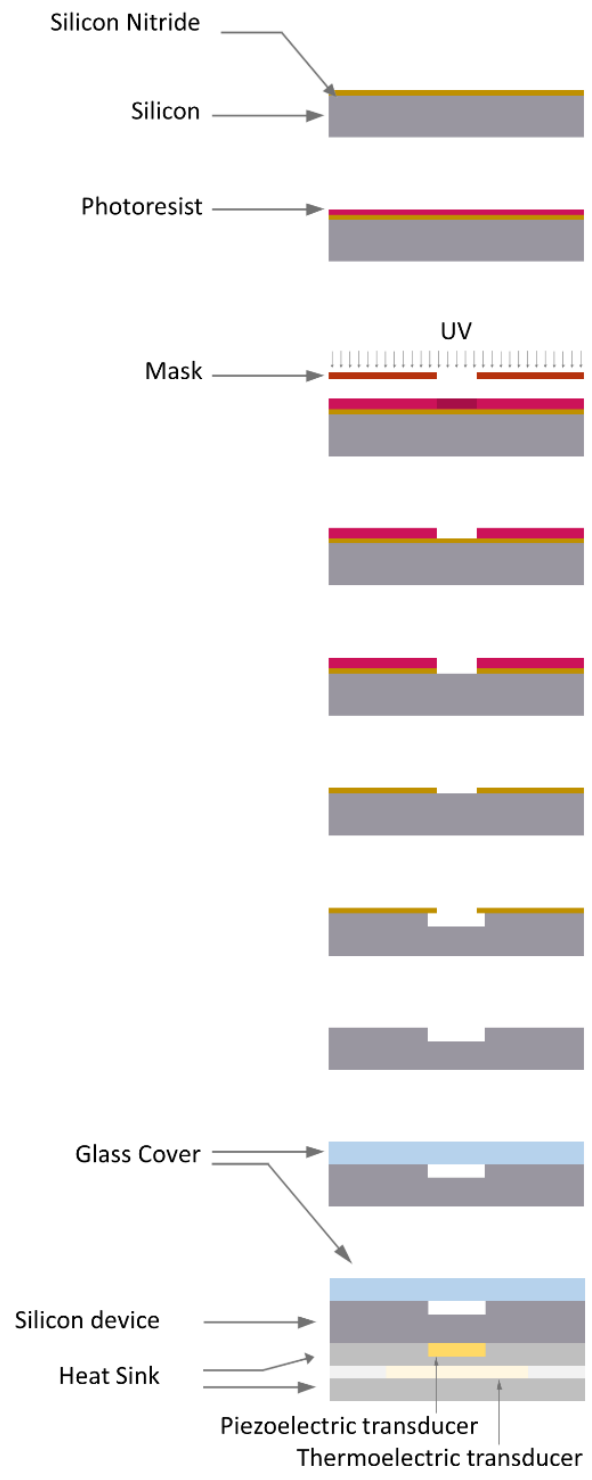


\subsection{Preparation of Vesicles}

Vesicles in this study prepared using a modified solvent-injection method in which the desired lipids in appropriate ratio were mixed and dissolved in a solvent. A desired fluorescent dye was also added to this solution. Then, DI water was added to the system dropwise while the solution stirred using a magnetic stirrer. A syringe pump (New Era Pump Systems, Inc.) was used to add water to the system under controlled rate of $10.0 \mathrm{ml} / \mathrm{h}$. the mechanism of vesicle formation is not fully known, however, the formation of small vesicles at lower water content due to the selfassembly properties and then the fusion of these small vesicles to form larger vesicles was reported as the formation mechanism of vesicles. ${ }^{150}$

This method is inspired by the ethanol-injection method, ${ }^{151,152}$ however, here tetrahydrofuran (THF) ( $\geq 99.9 \%$, inhibitor-free, Sigma-Aldrich) was used as the solvent. All the lipid containing THF solutions were prepared such that the concentration of the total lipids in THF was 1 to $3 \mathrm{wt} \%$. After water addition of about 25 to 35 volume $\%$ THF, the solvent was stored in room temperature overnight to allow the evaporation of THF solvent. Cholesterol and peptide addition to the system for samples that contained cholesterol or peptides were performed using the same method. Optical microscopy was used to examine the formation and size of the vesicles. Figure 2.4 shows fluorescent micrographs of the DMPC vesicles prepared with the described method. Optical micrographs and Image J software were used to measure the size of vesicles. The average size of vesicles prepared here were $1.41 \pm 0.20 \mu \mathrm{m}$. 

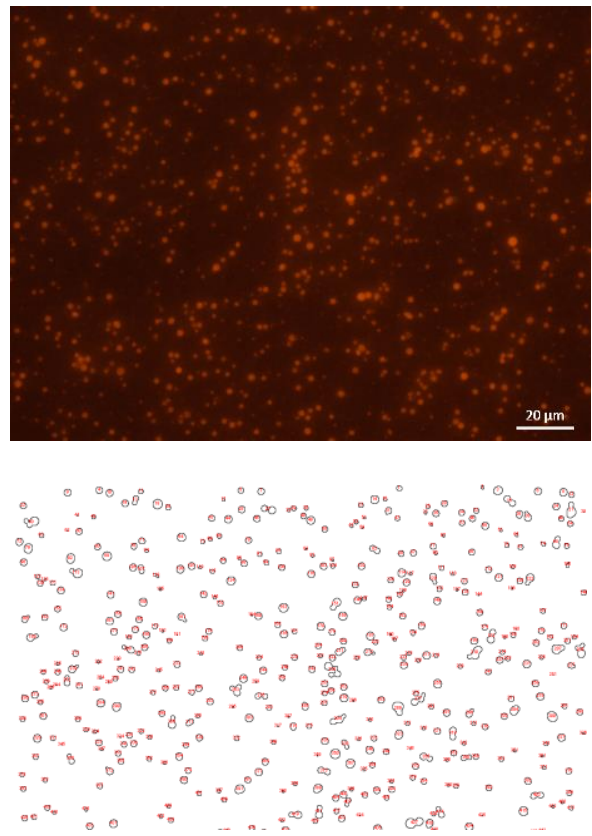

Average size $=1.32 \mu \mathrm{m}$
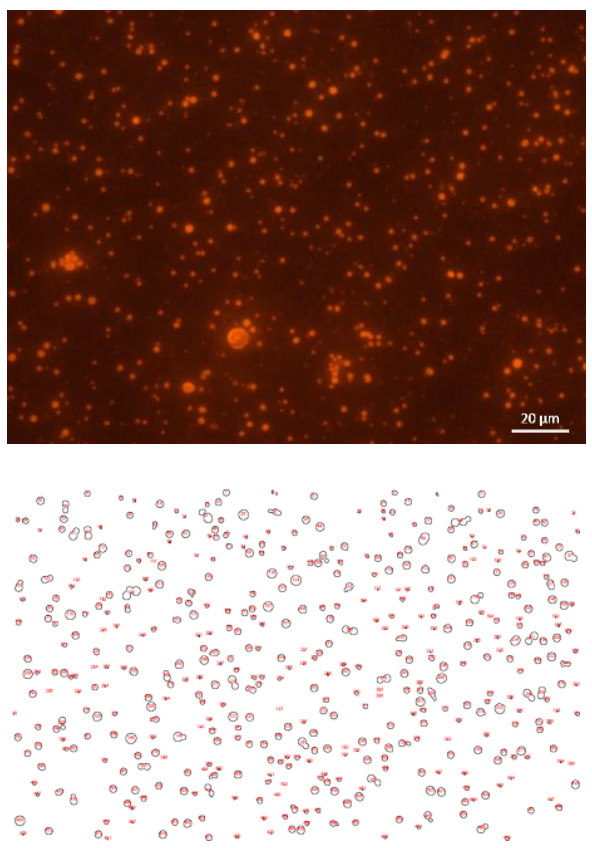

Average size $=1.50 \mu \mathrm{m}$

Figure 2-4 Optical micrograph of the DMPC vesicles prepared by the described method.

\subsubsection{Lipids}

The lipids used in this study for the preparation of vesicles were two different phosphotidylcholines (PCs) with linear saturated fatty acyl chains namely, 1,2-dimyristoyl-sn-glycero-3-phosphocholine (DMPC) and 1,2-dipalmitoyl-sn-glycero-3-phosphocholine (DPPC) with purities over 99\%. These lipids were purchased from Avanti Polar Lipids (Alabaster, AL) and used without any further purification. Cholesterol was purchased from Sigma-Aldrich (powder, BioReagent, $\geq 99 \%$ ). The reason for choosing these lipids was that these types of PCs were found in a number of biological membranes. And, therefore, these lipids were among the most investigated PCs in the literature for their mechanical and thermotropic behaviors. 


\subsubsection{Peptides}

In this study three different transmembrane peptides were used to better mimic the real condition of biological liposomes and investigate the influence of membrane protein content, particularly channel forming proteins, on the acoustophoretic behavior of vesicles. Of all the membrane proteins and peptides, pore forming peptides are the most extensively used ones for investigating the interactions of proteins with the membrane. ${ }^{35}$ They have also been used as model systems in various studies $^{17,153-155}$ aiming at evaluating the changes of membrane electrical properties upon the insertion of peptides. ${ }^{35}$ Gramicidin is one of the first commercially manufactured pore-forming peptide that is most frequently used as an antibiotic in model bilayer membranes. This antimicrobial peptide is in fact a mixture of pore forming peptides (gramicidin A 80\%, B 6\% and C 14\%), ${ }^{156}$ called collectively gramicidin D. ${ }^{35}$ Gramicidin D has a linear chain made of 15 amino acids, ${ }^{157}$ as opposed to gramicidin $\mathrm{S}$ that forms a cyclic peptide chain. ${ }^{158}$ Alamethicin is another channelforming peptide antibiotic, that can induce voltage-dependent ion channels in lipid membranes. ${ }^{159}$ Despite being made of only 20 amino acids, alamethicin exhibits channeling activity that is suggested to be relevant to that of physiological channel proteins which typically consist of 2,000 or more amino acids. ${ }^{160}$ Melittin is a peptide of 26 amino acids and is the main constituent of bee venom. Melittin molecule has both hydrophobic and hydrophilic parts, however, in an aqueous environment, it integrate itself into the cell membrane. Gramicidin D (99\%) was purchased from Fischer Scientific whereas Alamethicin ( $\geq 98.0 \%$, from Trichoderma viride) and Melittin were bought from Sigma-Aldrich. To prepare vesicles containing peptide, the desired concentration of peptide was added along with the lipid to the water-miscible solvent (THF).

\subsubsection{Labeling Dyes}

In order to better visualize the vesicles during the experiments in this study, fluorescent dyes were used to label the vesicles. The selected dyes were lipophile so that they tag only the lipid membrane of the vesicles. One of the fluorescent dyes was Nile red (9-diethylamino-5-benzo- 
$[\alpha]$ phenoxazinone) and was purchased from Acros Organics. This dye is a red-fluorescent dye and does not produce any interaction with the lipid it is tagged to. ${ }^{161}$

Laurdan (6-dodecanoyl-2-dimethylaminonaphthalene) and DiOC6(3) iodide [3,3dihexyloxacarbocyanine iodide], which are blue-fluorescent and green-fluorescent dyes respectively and were also bought from AnaSpec, Inc, were the other dyes used in this study. The structure of these fluorescent dyes is also such that they render a hydrophobic affinity and therefore they locate inside the membrane. 
3 The Effect of Proteins on Thermo-Acoustofluidic properties of Vesicles

\subsection{Introduction}

Cells rely on vesicles to exchange information with other cells, dispose of their waste, modify their environment, and perform many other specialized functions. Various types of biomolecules such as nucleic acids, lipids, and proteins are enclosed in the vesicles before secretion into the extracellular milieu. ${ }^{162}$ Examining the biochemical composition of these "extracellular vesicles" (EVs) would therefore provide valuable insights into the state of the parent cells and the biology mediated by these EVs. ${ }^{163}$ For instance, the genomic analysis of vesicles shed from cancer cells contains signature features of the underlying pathology, ranging from the parent cells' phenotype, progression of the disease, and responsiveness to treatment. ${ }^{19,22,24}$ Likewise, the proteomic analysis of EVs released by immune cells has revealed an important role for these vesicles in tuning immune responses to inflammations, tumors, and pathogens. ${ }^{164}$

Informative contents along with a relatively crowded existence in all biological fluids have highlighted EVs as promising sources of biomarkers. ${ }^{165-170}$ There is consequently a growing interest in studying EVs to develop liquid biopsy platforms for monitoring health and disease development. ${ }^{171,172}$ This however has been hindered by technical limitations in the isolation of EVs. ${ }^{19,24}$ One particular challenge is that existing methods such as differential centrifugation and ultrafiltration can merely discriminate between vesicles based on their size and are not based on their composition or cells of origin. ${ }^{19,22,24,173}$ Likewise, microfluidic-based methods, despite achieving high efficiencies, can only indiscriminately trap vesicles. ${ }^{174,175}$ In fact, even more sophisticated methods like immunoaffinity-based capture ${ }^{176,177}$ cannot differentiate between vesicles with different contents of the same protein. Therefore, complementary techniques such as mass spectrometry, flow cytometry, and microscopy are often required to further characterize the separated vesicles. 
Of all the parameters based on which vesicles could be differentiated, composition is perhaps the most reliable indicator of their identity. The term composition in this context refers to the multitude of compounds that make up the lipidome and proteome of an EV along with its genetic material. Not only composition can be used as a separation criterion, but in fact the changes in composition can also be considered as a biomarker. For instance, lipid profiling of the membrane of EVs isolated from prostate cancer cells shows an enrichment in glycosphingolipids, cholesterol, sphingomyelin and phosphatidylserine in the membrane. ${ }^{178}$ Similarly, EVs secreted from colorectal cancer cells shows an enrichment of specific lipid classes including sphingolipids, sterol lipids, glycerolipids and glycerophospholipids and particularly plasmalogen and alkyl ether containing glycerophospholipids in the membrane. ${ }^{179}$ Such enrichment or depletion of a specific lipid or protein can be regarded as a biomarker associated with an underlying condition. ${ }^{173}$ Therefore, a reliable method that can rapidly and reliably differentiate and sort EVs based on their composition would be of great significance in biomarker discovery and biomedical diagnostics.

Standing wave acoustophoresis has been previously demonstrated for separations consisting of a variety of different microparticles ranging from synthetic droplets ${ }^{180}$ to cancer cells, white and red blood cells and even proteins. ${ }^{125,147}$ More recently, enrichment of EVs from biological fluids was achieved using tilted-angle standing sequential surface acoustic wave (taSSAW) ${ }^{181}$ and beadmediated acoustic trapping. ${ }^{182-184}$ As was mentioned in Section 1.3, separation in acoustophoresis is mainly realized through the size- and/or density-based differences in the primary acoustic radiation force exerted on the particles, described by Equation (1-11), in which, generation of a large enough force causes the migration of particles along directions determined based on the sign of $\Phi$, where positive values afford nodal migration and negative values afford anti-nodal migration. ${ }^{125}$ Acoustophoresis inherently stands out as a desirable candidate for cell separation applications, as it is label-free, fast, and non-invasive. Nonetheless, the fact that most cells often do not vary much in density, makes acoustophoresis of same-size cells and vesicles a challenging 
task. Furthermore, almost all membrane-bound systems such as cells and vesicles are found to be indifferentiable based on only compressibility at room temperature.

We previously demonstrated that lipid vesicles can undergo a reversible nodal or anti-nodal migration in a thermally-modulated ultrasonically-stimulated microfluidic channel. ${ }^{185}$ The switch from nodal to anti-nodal focusing was determined to occur at a specific transition temperature attributed to a biomechanical transition in the stiffness of vesicles as the lipid membrane undergoes a thermotropic transition from an ordered "gel" phase to a disordered "fluid" phase. Since thermotropic transitions are composition-specific, the temperature at which this switch is observed is accordingly specific to the lipid composition. This specificity was put to the test for the separation of vesicles with various phospholipid compositions, ${ }^{185}$ various cholesterol contents, ${ }^{186}$ and was used for the sorting of vesicle mixtures of up to three vesicle systems. ${ }^{186}$ The differentiation, however, has been mainly dictated by the membrane lipidome, while for medically relevant vesicles proteins embedded in the membrane play a major role. ${ }^{55}$ In fact, membrane proteins have been recently suggested to be the main contributor to the stiffness of natural EVs. ${ }^{56}$ The over and underexpression of certain proteins in extracellular vesicles has been observed in many physiological and pathological conditions; however, a simple method to sort vesicles based on contrast in protein content is yet to be developed.

We herein present a non-affinity-based method for rapid and inexpensive isolation of vesicles based on their membrane protein content. Based on a composition-specific thermophysical property change of vesicles at various protein contents, an acoustic property change that enabled an acoustophoretic separation was observed. ${ }^{187}$ This change was demonstrated in a thermallymodulated acoustofluidic device in the form of a shift in vesicle migration from the nodal plane to antinodal plane at a specific temperature known as the acoustic contrast temperature $\left(T_{\Phi}\right)$. In this section, we examine the influence of membrane protein content (molar ratio) of particularly 
channel-forming proteins, on the acoustophoretic behavior of vesicles. We focus on pore forming peptides since they are some of the most extensively studied in membrane ${ }^{35}$ and have also been used as model systems in various other studies. ${ }^{17,153-155}$ Figure 3.1 shows the schematic of the separation device fabricated using the previously described microfabrication technique in section

\section{2 .}

The peptides selected for this study are gramicidin $\mathrm{D}$, an antimicrobial linear peptide made up of 15 amino acids, alamethicin, another antimicrobial linear peptide made up of 20 amino acids, and melittin, a small protein made up of 26 amino acids and the principal toxin in the bee venom. Using phosphatidylcholine vesicles containing the membrane proteins gramicidin D and alamethicin at molar contents ranging from $1 \%$ to $10 \%$, we observed that increasing the membrane protein content brought about conformational changes in the membrane which afforded the vesicles distinctive acoustic properties.

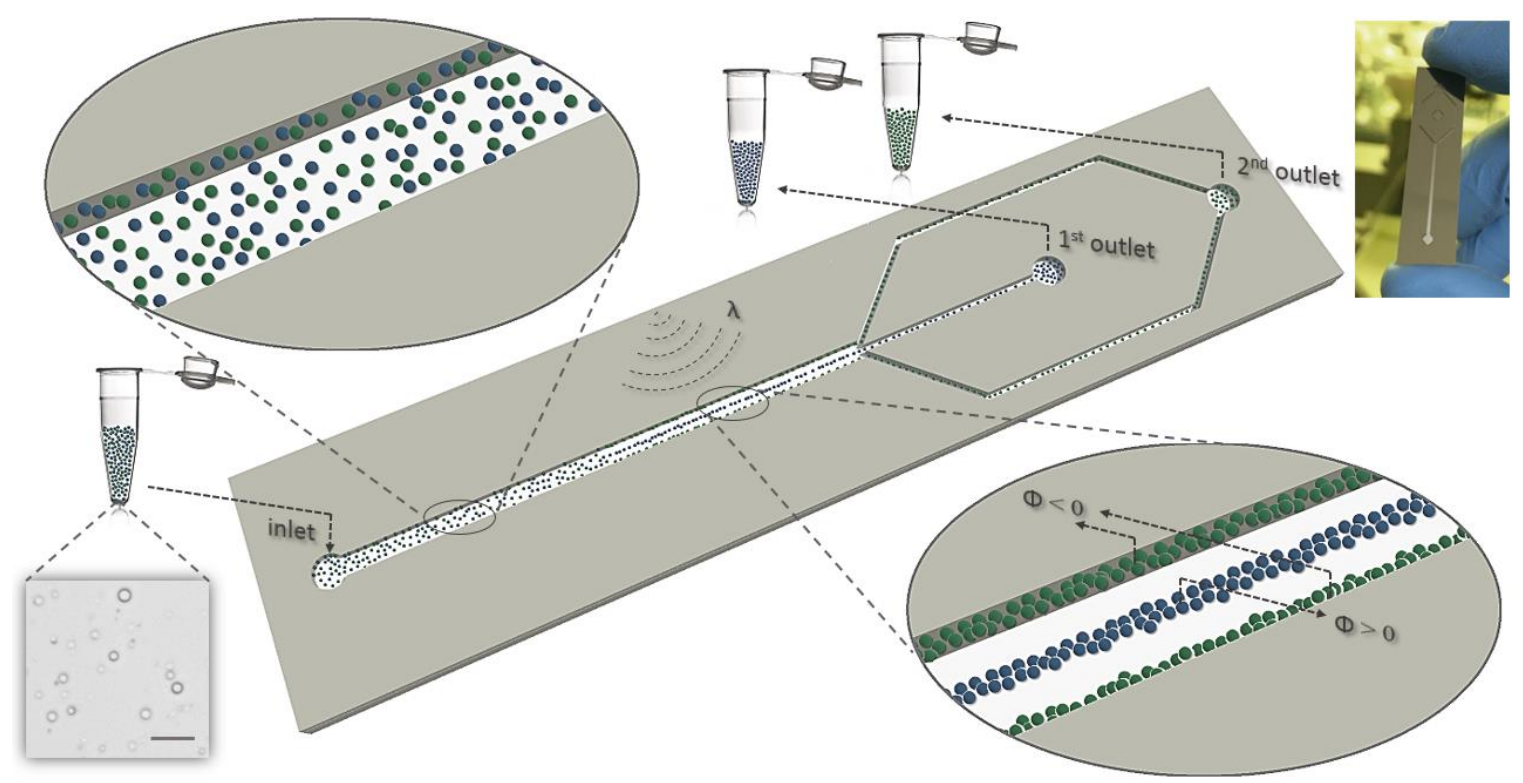

Figure 3-1 Schematic of the acoustofluidic device in a separation experiment; two types of EVs injected through the same inlet initially flow randomly but upon actuation of the device at a specific frequency and temperature focus in distinct streamlines running along different outlets. The number and position of streamlines is determined by the frequency or wavelength $(\lambda)$ of the actuation signal which is in turn adjusted according to the width of the channel in the separation region. Inset on the left shows an 
optical micrograph of the vesicles with a scale bar of $10 \mu \mathrm{m}$. The image on the right is the actual microfluidic chip.

Then by establishing an acoustic contrast temperature window, vesicles with same protein but different molar content were successfully separated. The efficiency of the separation was studied for various vesicle mixtures and a separation efficiency as high as $97 \%$ was accomplished. This method holds promise for myriad applications in the biomedical field. The fractionation of extracellular vesicles based on the expression level of different proteins would be of high significance in medical diagnostic, therapeutic, and drug discovery applications.

\subsection{Transmembrane Peptides: Gramicidin, Alamethicin and Melittin}

Gramicidin is one the first commercially manufactured antibiotics. This antimicrobial peptide that is in fact a mixture of pore forming peptides (gramicidin A $80 \%$, B $6 \%$ and C $14 \%$ ) ${ }^{156}$, and called collectively gramicidin $\mathrm{D}$ has been used in different studies to investigate the interaction of peptides with the membrane. ${ }^{35}$ These pore forming peptides have also been acting as model systems in various studies ${ }^{17,153-155}$ aiming at evaluating the changes of membrane electrical properties upon insertion of peptides ${ }^{35}$. These peptides are produced by soil bacterial species Bacillus brevis and are active against Gram-positive bacteria except for the Gram-positive bacilli against selected Gram-negative organisms, such as Neisseria bacteria ${ }^{158}$. Gramicidin D has a linear chain made of 15 amino acids ${ }^{157}$, opposed to gramicidin $S$ that forms a cyclic peptide chain ${ }^{158}$. Gramicidin D create active pores/channels in lipid membrane to enhance the transport of small cations by inserting into the hydrophobic region of membranes ${ }^{188-192}$. Figure 3.2 shows schematic of building blocks of a bilayer membrane containing PC lipid molecules such as DPPC and transmembrane peptides such as gramicidin D. It was shown that the chain melting transition of lipids can be

changed upon insertion of peptides and proteins ${ }^{158,193}$. This changes in transition profile can be observed either as broadening and/or shifting the curve to lower or higher temperatures. In the case of gramicidin S a shift to lower temperatures was observed ${ }^{158,193}$. Zein et al. ${ }^{194}$ also asserted that 
the addition of gramicidin into the DMPC bilayer has an acute effect on the lipid bilayer structure and temperature, pressure phase behavior ${ }^{158,194}$. Additionally, the insertion of peptide or proteins in the membrane has shown to influence the dynamic structure of the bulk lipid. ${ }^{158,195}$ The other peptide that was used in this study is alamethicin, which is a 20 -residue peptide antibiotic that promotes voltage-dependent ion channels in lipid membranes as it is almost entirely hydrophobic. ${ }^{159}$ The addition of alamethicin into the membrane causes the hydrophobic regions of the membrane to try to match the hydrophobic regions of peptide. This hydrophobic matching induces some long-range deformation in the configuration of the bilayer. Overlapping these deformation regions decreases the deformation free energy of the system. ${ }^{160}$

a) Building blocks

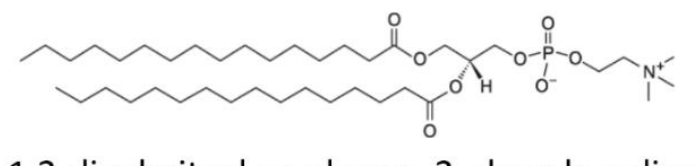

1,2-dipalmitoyl-sn-glycero-3-phosphocoline (DPPC)

b) Liposome
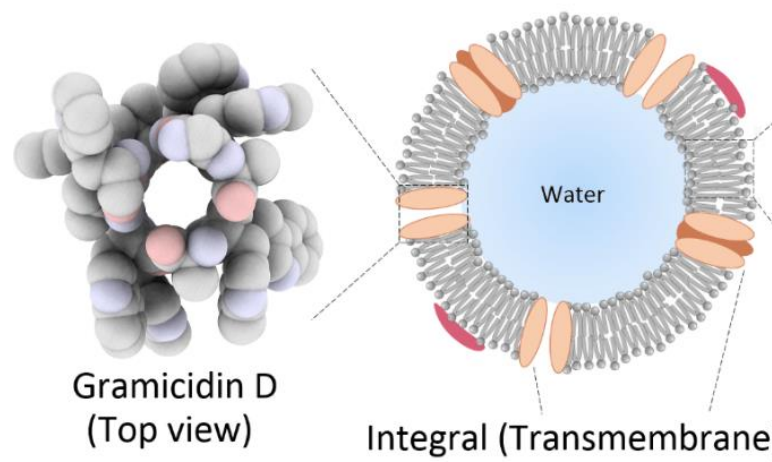

Integral (Transmembrane) protein
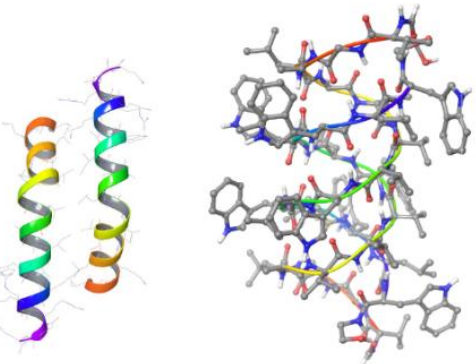

Transmembrane protein

(Gramicidin D)

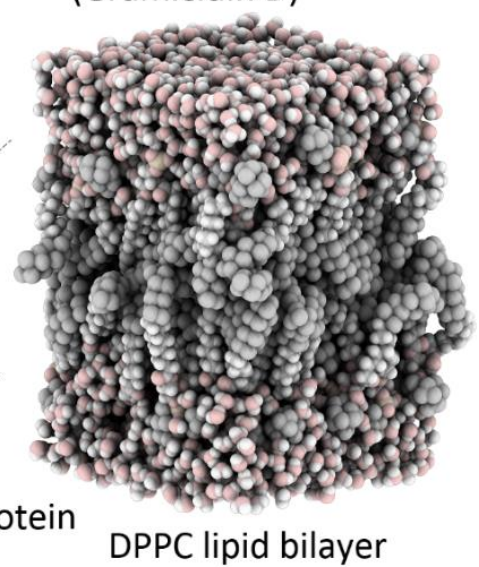

Figure 3-2 (a) The building blocks of the lipid vesicles used in this study: amphiphilic lipids (e.g. DPPC), and transmembrane peptides (e.g. Gramicidin D); (b) Upon hydration under proper conditions, the lipids arrange in bilayers to self-assemble to form liposomes of average $1 \mu \mathrm{m}$ incorporating transmembrane peptides which, depending on their type and concentration, can assume various conformations (e.g. transmembrane pores) in the membrane. 


\subsection{Thermo-Acoustofluidic Behavior Vesicles Containing Model Proteins}

The workflow of thermally-assisted acoustophoresis begins by examining the existence of an acoustic contrast temperature, $T_{\Phi}$, for each vesicle composition. Mixture of vesicles with different compositions will yield two distinct $T_{\Phi}$ values that will constitute the separation temperature window. Operating the device at a temperature within that window yields an opposite response for each vesicle composition, namely nodal and anti-nodal, thus achieving a separation. PC vesicles with various amounts of gramicidin $\left(X_{\mathrm{g}}\right)$ and alamethicin $\left(X_{\mathrm{a}}\right)$ and melittin $\left(X_{\mathrm{m}}\right)$ ranging from 0 to $10 \mathrm{~mol} \%$, were first individually injected into separate channels to determine their $T_{\Phi}$ values. Prior to the injection of vesicles, the first harmonic frequency $\left(f_{1}\right)$, which produces one nodal plane in the center of the channel and two antinodal planes at the walls, was pre-determined using the initial conditions. At $f_{1}$ and $15{ }^{\circ} \mathrm{C}$, a prompt nodal focusing was expectedly observed for all vesicle systems. A controlled heating was then initiated and at $T_{\Phi}$ all vesicles in the channel collectively proceeded to migrate from the centre towards the walls of the channel. That specific temperature was recorded for five separate preparations of identical compositions, and the average was identified as $T_{\Phi}$ and was recorded for each vesicle composition. The $T_{\Phi}$ data collected from these experiments are plotted in Figures 3.3, 3.4 and 3.5 depicting how the presence of proteins distinctly affects the $T_{\Phi}$ of the vesicles.

All $T_{\Phi}$ values recorded here, like other systems investigated in our previous studies, were found to be specific to the composition of each system. This specificity can be explained by the distinctive effects of proteins on the membrane mechanics which have been investigated in various studies. While some studies have reported a significant increase in the bending modulus $\left(k_{c}\right)$ of vesicles reconstructed from yeast membranes ${ }^{50}$ and influenza viruses ${ }^{51}$ due to the presence of membrane proteins, others have shown either a neutral effect ${ }^{52}$ or a decrease in the $k_{c} \cdot{ }^{53,54,196}$ The reason for this lies in the fact that transmembrane proteins, depending on their concentration, can assume different conformations in the membrane. ${ }^{191,197,198}$ For instance, for many pore-forming proteins a 
threshold concentration, typically shown as the molar ratio of polypeptide or protein to lipid $\left(\mathrm{P} / \mathrm{L}^{*}\right)$, can be identified for the pore-forming activity of the protein in membrane. Below $\mathrm{P} / \mathrm{L}^{*}$, the protein or peptide is randomly dispersed in the membrane showing no pore-forming activity, but as the concentration is increased above the $\mathrm{P} / \mathrm{L}^{*}$ a specific conformation is adopted by the protein leading to the formation of pores across the membrane. ${ }^{199}$

In the case of gramicidin, as evident in Figures 3.3a and b, the random incorporation of peptide in the membrane first increases the order parameter in the hydrophobic region of the membrane ${ }^{200}$ and therefore stiffens the membranes. This stiffening is manifested as a $1{ }^{\circ} \mathrm{C}$ increase in the $T_{\Phi}$ of systems with $X_{g}=1 \%$, compared to respective pure systems.

As more peptide is added to the membrane, an upright pore-forming conformation is adopted by the protein which is typically accompanied by a size mismatch in the local membrane enclosing the protein. This mismatch, which is defined as the difference between the thickness of the hydrophobic moieties on the transmembrane peptide and the surrounding lipid bilayer, ${ }^{201}$ causes a local thinning or thickening in the bilayer and therefore weakens the membrane. For DMPC and DPPC, the thickness of the hydrocarbon part of the pure lipid $\left(h_{0}\right)$ is sufficiently larger (approximately $25 \AA$ and $27 \AA,{ }^{202}$ respectively) than the length of the hydrophobic part of the channel $\left(h_{\mathrm{G}}\right)$, which is $\sim 22 \AA,{ }^{191}$ rendering a negative mismatch and a local bilayer thinning in the bilayer. ${ }^{197}$ This softening (reduction in stiffness) appears as a decrease in the rate by which the $T_{\Phi}$ increases in the case of DMPC system and an actual decrease in $T_{\Phi}$ for the DPPC system.

Transmembrane proteins, usually at higher concentrations, can once again adopt a non-poreforming conformation and even induce structural changes in the membrane. ${ }^{35}$ Gramicidin, for instance, can transform from dimer channels spanning the membrane, ${ }^{191}$ to dissociated aggregates ${ }^{203,204}$ once its increased presence is no longer accommodated by the membrane. This could explain the increase in $T_{\Phi}$ in both DMPC and DPPC systems where the breaking of the dimer 
channels and its consequent increase in the amount of aggregated peptide leads to an increase in the $k_{c}$ and accordingly an increase in the $T_{\Phi}$ up to $10 \mathrm{~mol} \%$.

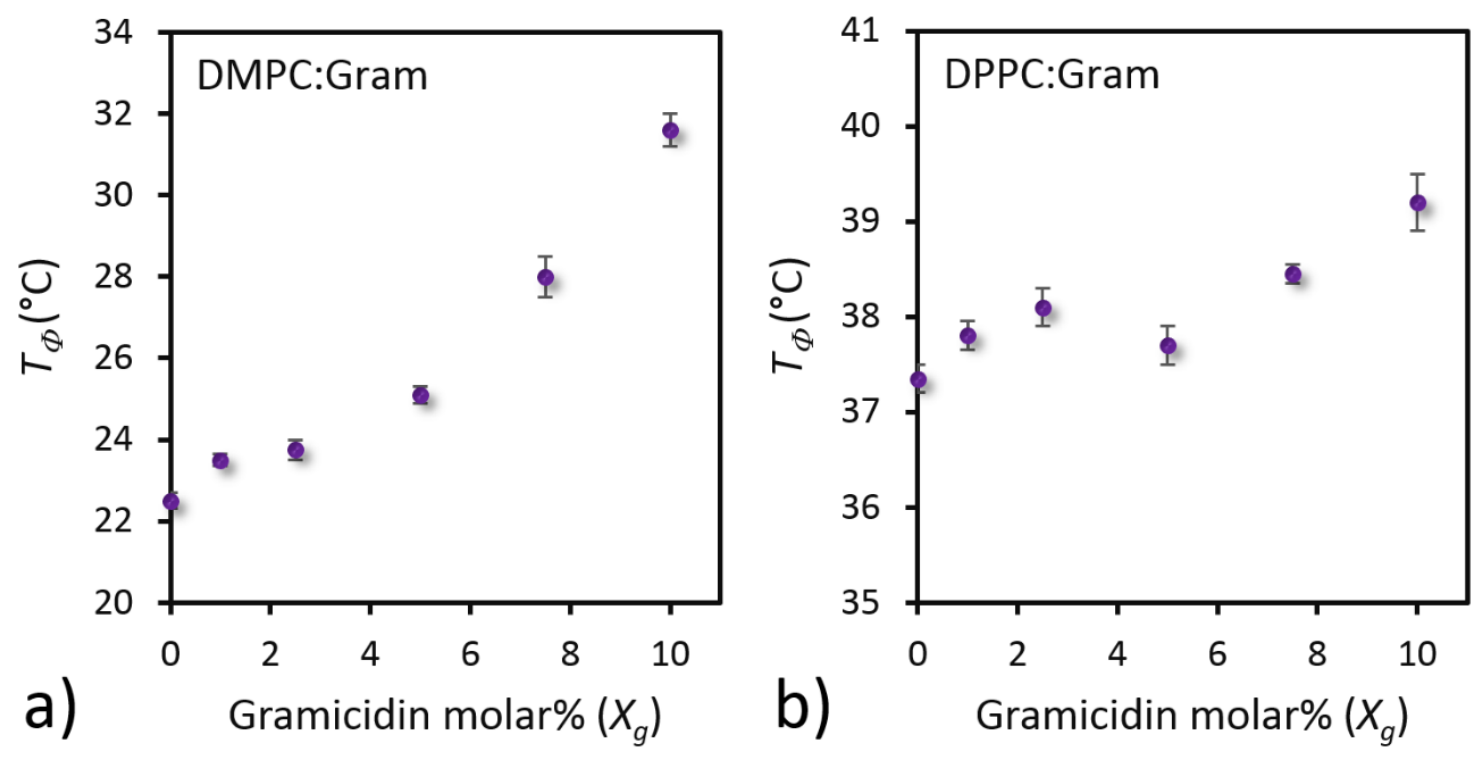

Figure 3-3 The acoustic contrast temperature, $T_{\phi}, v s$. composition for (a) DMPC and (b) DPPC vesicles, upon the introduction of gramicidin. The error bars represent the maximum and minimum values recorded for each system in at least five independent experiments using separate preparations.

In the case of alamethicin, a polypeptide known to have significant influence on the $k_{c}$ of lipid membranes, ${ }^{196}$ a sharp decrease in $T_{\Phi}$ is observed for both DMPC and DPPC systems (Figures 3.4a and b). This can be explained by considering the fact that the hydrophobic thickness of alamethicin, which is $27-28 \AA{ }^{198}$ is greater than both DMPC and DPPC bilayers and therefore with increasing peptide concentration a progressive thickening occurs in the membrane that decreases the $k_{c}$. The resulting softening is evident through the decline in the $T_{\Phi}$ at lower peptide contents in both systems. This decline, which in the case of DMPC appears to be continuous up to $X_{\mathrm{a}}=10 \%$, is followed by a moderate increase in the DPPC system at $X_{\mathrm{a}}=2.5 \%$, possibly due to the aggregation of peptide in the membrane at higher concentrations. 


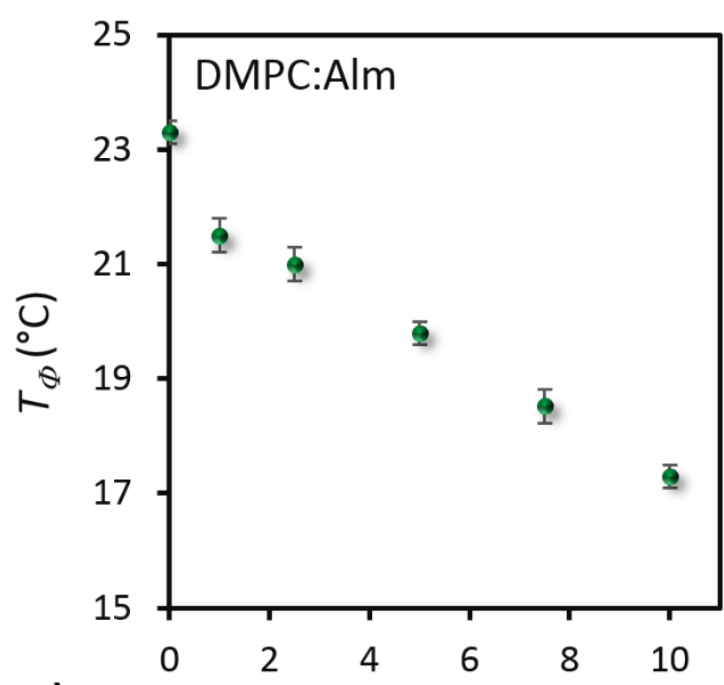

a)

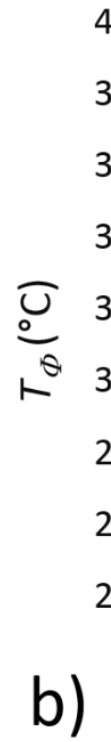

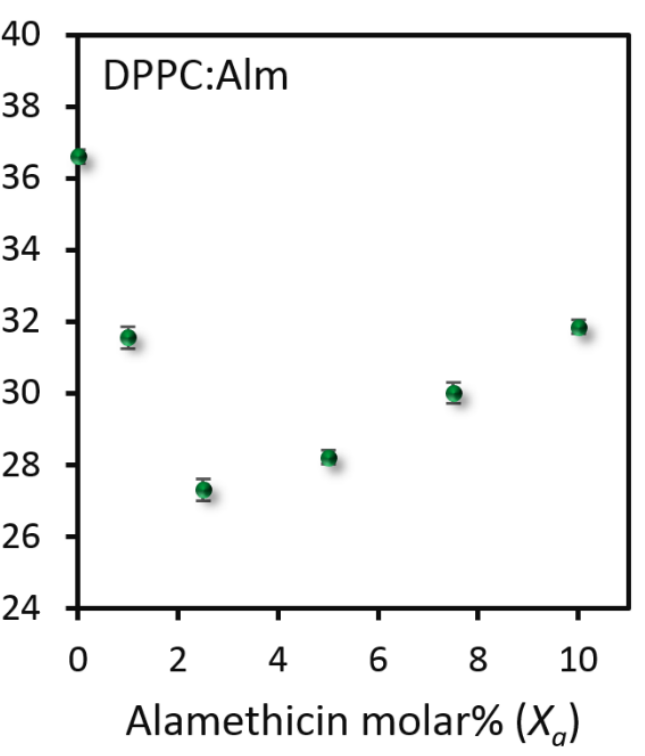

Figure 3-4 The acoustic contrast temperature, $T_{\infty}$, vs. composition for (a) DMPC and (b) DPPC vesicles, upon the introduction of alamethicin. The error bars represent the maximum and minimum values recorded for each system in at least five independent experiments using separate preparations.

Melittin also disrupts the membrane and produces pores similar to those formed by alamethicin. ${ }^{205}$ Compared to other peptides with 8-26 amino acids, melittin dramatically influences the membrane's order, ${ }^{206}$ causing a sharp decrease in its bending rigidity. ${ }^{207}$ This phenomena is shown in Figs. 3.5a and b, when introducing small amounts of melittin $\left(X_{m}=0.1 \%\right)$ to DMPC and DPPC lower the $T_{\Phi}$ of the system by 7.5 and $6.5^{\circ} \mathrm{C}$, respectively. This is due to the partitioning and agglomeration of peptides and the disruption of the membrane structure due to the peptide-induced pore formation.

In general, the temperature at which the migration towards the antinodal planes is initiated, is recorded as the $T_{\Phi}$. This point as discussed in our previous studies ${ }^{185,186}$ does not necessarily correspond to any thermotropic transition(s) in the respective system. It however reflects the moment at which the $\Phi$ assumes a non-positive value, in the case of heating. In fact, when recorded accurately, a small plateau can be observed in the temperature as heating continues with a constant rate to a point close to the thermotropic transition of the system (Figure 3.6). 

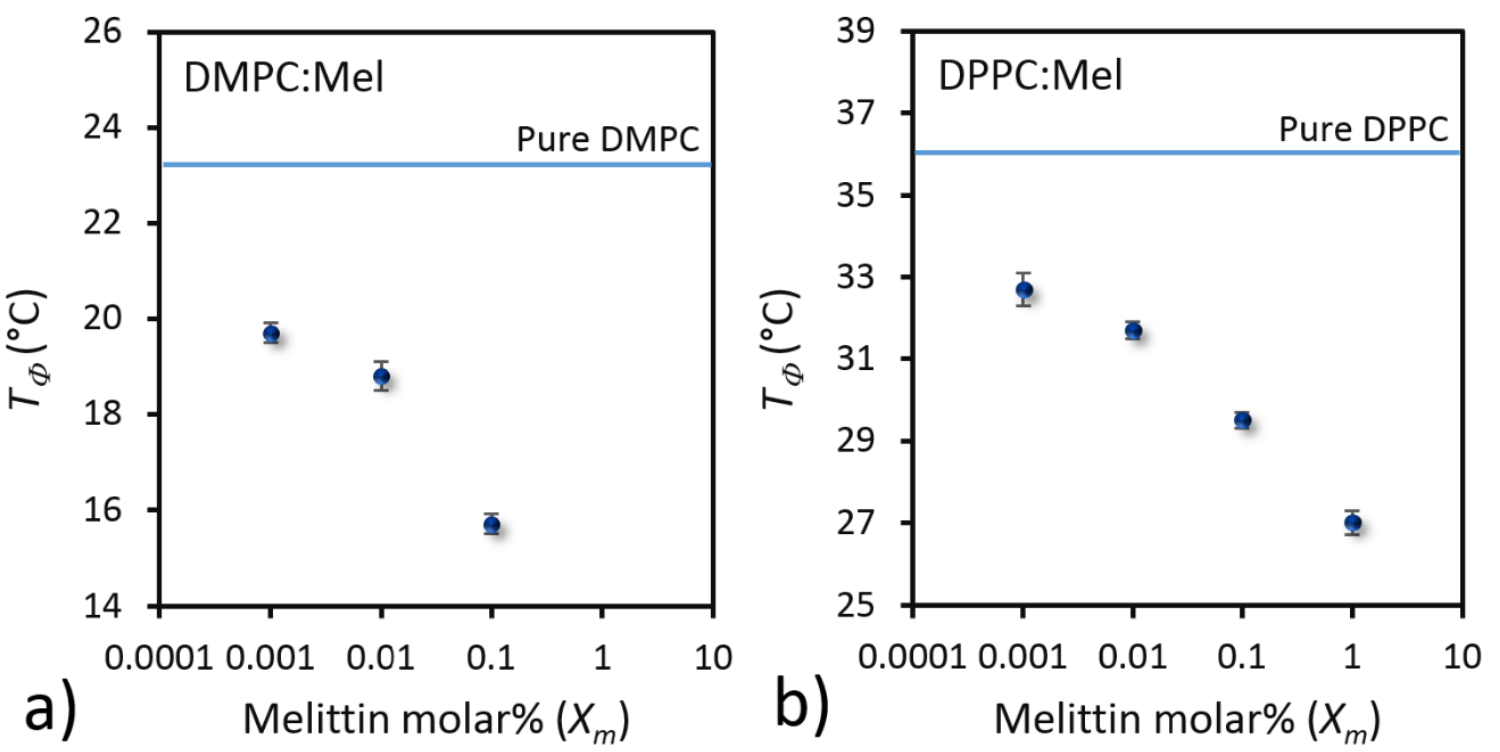

Figure 3-5 The acoustic contrast temperature, $T_{\phi}, v s$. composition for (a) DMPC and (b) DPPC vesicles, upon the introduction of melittin. The error bars represent the maximum and minimum values recorded for each system in at least five independent experiments using separate preparations. The blue horizontal line indicates the $T_{\phi}$ of pure DMPC and DPPC systems.

This drop or gap in the temperature rise can be attributed to the heat absorbed by the vesicles as they undergo the endothermic transition from the more ordered gel phase to the rippled phase $\left(\mathrm{L}_{\beta}\right.$, $\rightarrow \mathrm{P}_{\beta}$ ) or from the rippled phase to the fluid phase $\left(\mathrm{L}_{\beta}, \rightarrow \mathrm{L}_{\alpha}\right)$. When the heating rate is faster, this gap is not as evident as it is in slower heating regimes. In all cases, however, $T_{\Phi}$ was according to its definition identified as the onset of the change in the focusing behavior, not the point at which the plateau is observed.

\subsection{Separation of Vesicles Based on Protein Molar Ratio $\left(X_{\text {protein }}\right)$}

The main objective of this study is to demonstrate the reliable separation of vesicle subpopulations which contain vesicles of similar size but of different protein composition. The difference in composition, as mentioned before, can be due to the source cells from which vesicles originate or the variations in lipidomic and proteomic composition conferred on the vesicles by an underlying condition. ${ }^{20,208}$ To investigate the separation of subpopulations which have vesicles with varying expression levels of certain proteins, we chose two DPPC vesicles with different molar ratios of 
gramicidin $\left(X_{g}=7.5 \%\right.$ and $\left.X_{g}=10 \%\right)$. The vesicles were respectively fluorescently labelled with green and blue and then mixed just prior to being injected into the microfluidic device. When the acoustic actuator was switched off at $T=37^{\circ} \mathrm{C}$, all the vesicles were randomly dispersed (Figure 3.7a).

After actuation $\left(f_{1}=1.4 \mathrm{MHz}, 50 \mathrm{~V}_{\mathrm{pp}}\right)$ at the same temperature, vesicles of both compositions proceeded to migrate toward the central nodal plane (Figure 3.7b). The nodal migration for both vesicles system was expected, since the temperature was below the $T_{\Phi}$ values for both vesicles. At $39^{\circ} \mathrm{C}$, since the temperature now exceeded the $T_{\Phi}$ of $X_{g}=7.5 \%$ vesicles, these vesicles swiftly proceeded to migrate toward the antinodal planes at the walls (Figure 3.7c).

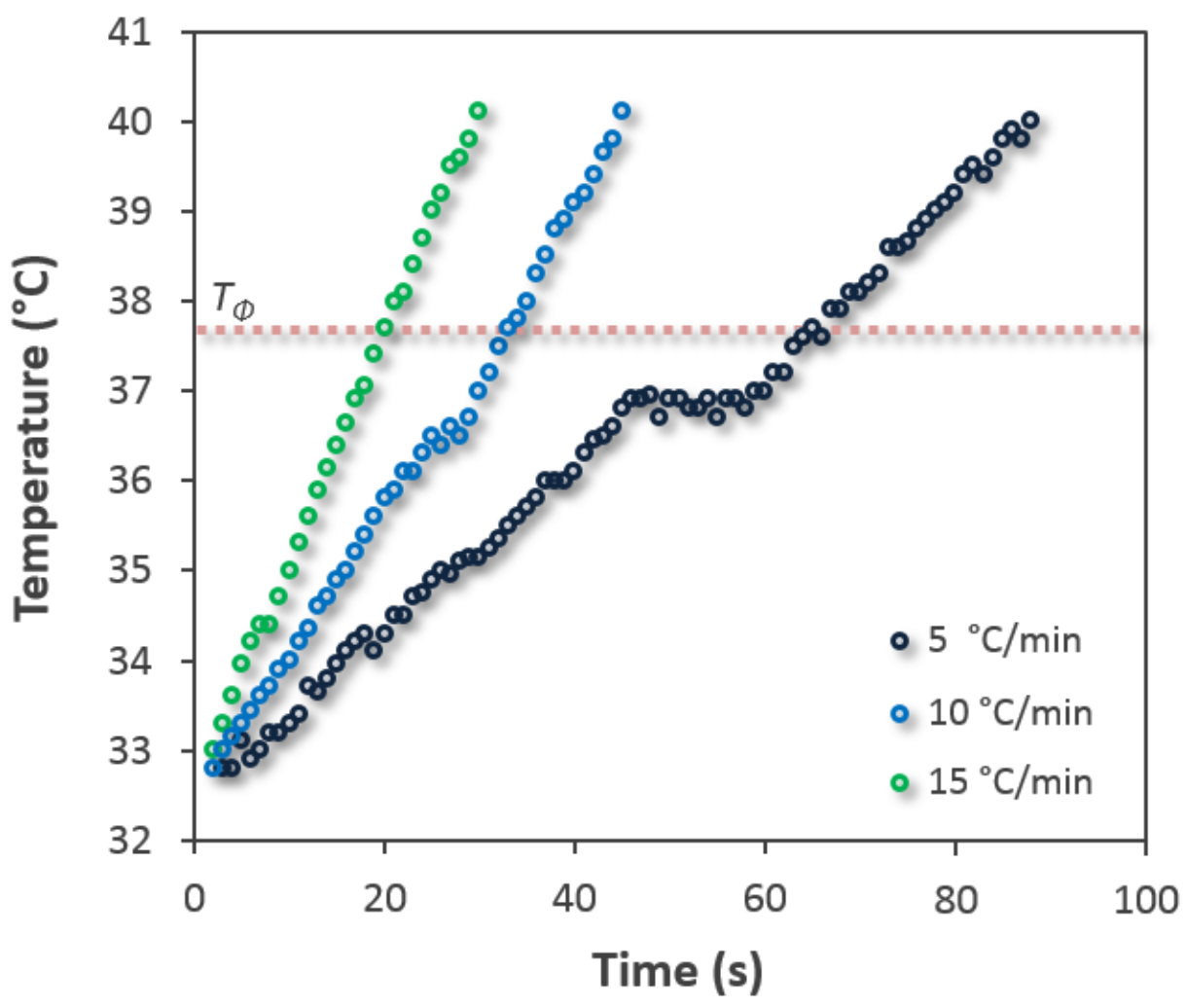

Figure 3-6 Recording of temperature vs. time under different heating rates $\left(5,10\right.$ and $\left.15{ }^{\circ} \mathrm{C} \cdot \mathrm{min}^{-1}\right)$ when measuring $T_{\Phi}$ of DPPC vesicle systems with $\mathrm{Xg}=1 \%$. A $1^{\circ}$ difference can be detected between the point at which the stagnation phase is observed and the point at which the antinodal migration is initiated $\left(T_{\Phi}\right)$. The plateau, evident in $5{ }^{\circ} \mathrm{C}$ min $^{-1}$ heating rate, is not as evident in $10{ }^{\circ} \mathrm{C} \mathrm{min}{ }^{-1}$ and is completely absent in faster heating rates $\left(15^{\circ} \mathrm{C} \mathrm{min}^{-1}\right)$. 

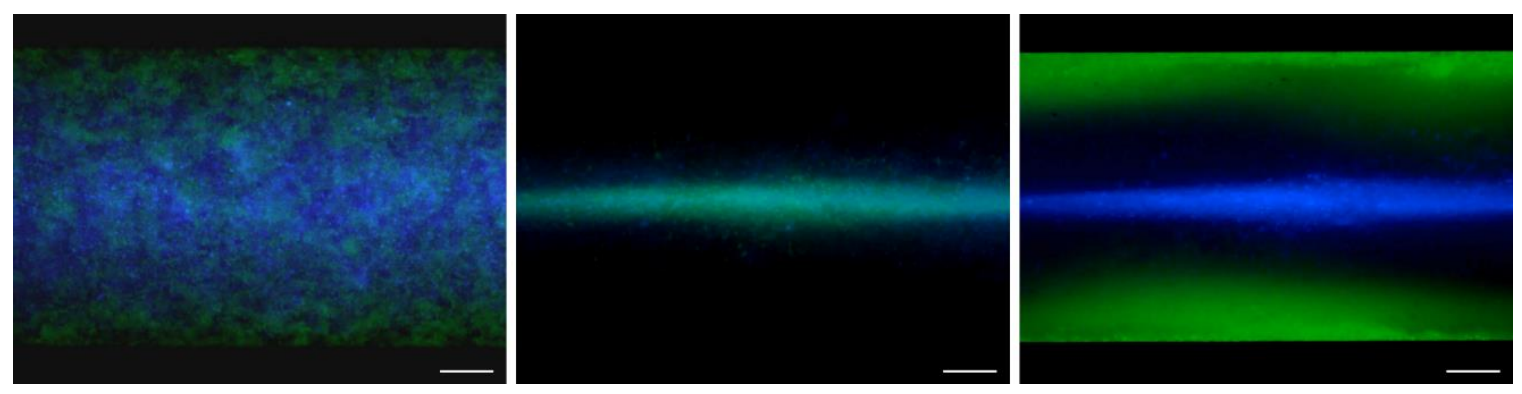

Figure 3-7 Fluorescence micrographs of the experiment involving the separation of DPPC vesicles with $X_{g}=7.5$ (green) and $X_{g}=10 \%$ (blue). (a) Vesicles are randomly dispersed in the microchannel at $37{ }^{\circ} \mathrm{C}$ with no acoustic actuation. (b) The same vesicles in figure (a) 3 seconds after setting the acoustic transducer to the first harmonic frequency undergo a nodal focusing at the same temperature. (c) After sweeping up the temperature to $\sim 39^{\circ} \mathrm{C}, X_{g}=7.5$ vesicles (green) begin to migrate toward the antinodes while $X_{g}=10 \%$ vesicles (blue) remain at the pressure node of the channel (scale bar in all images is 100 $\mu \mathrm{m})$. Vesicles now flowing along distinct streamlines can be collected from separate outlets.

Since the separation temperature remained lower than the $T_{\Phi}$ of $X_{g}=10 \%$, these vesicles remained focused at the central node and were gradually collected from the middle $\left(1^{\text {st }}\right)$ outlet.

A separation criterion could also be developed a priori based on the differences in $T_{\Phi}$ of vesicles by calculating the value of $\Phi$ at different temperatures. Figure 3.8 shows one such plot obtained by calculating the relative values of $\Phi$ using the compressibility data of the same compositions studied here. The compressibility data were obtained from the literature. ${ }^{158}$ In this plot, $T_{\Phi}$ for each system corresponds to the point at which the $\Phi$ crosses the temperature axis $(\Phi=0)$ for that system. The range between the crossing points of any two $\Phi$ curves on the abscissas determines the separation "window" for the acoustophoresis, shown as a blue band for the $X_{\mathrm{g}}=7.5 \%$ and $X_{\mathrm{g}}=10 \%$ systems. If the chip temperature is set to stay within this window, vesicles with $T_{\Phi}$ greater than this temperature will migrate to the nodes, while the ones with $T_{\Phi}$ below this temperature will migrate to the antinodes. 


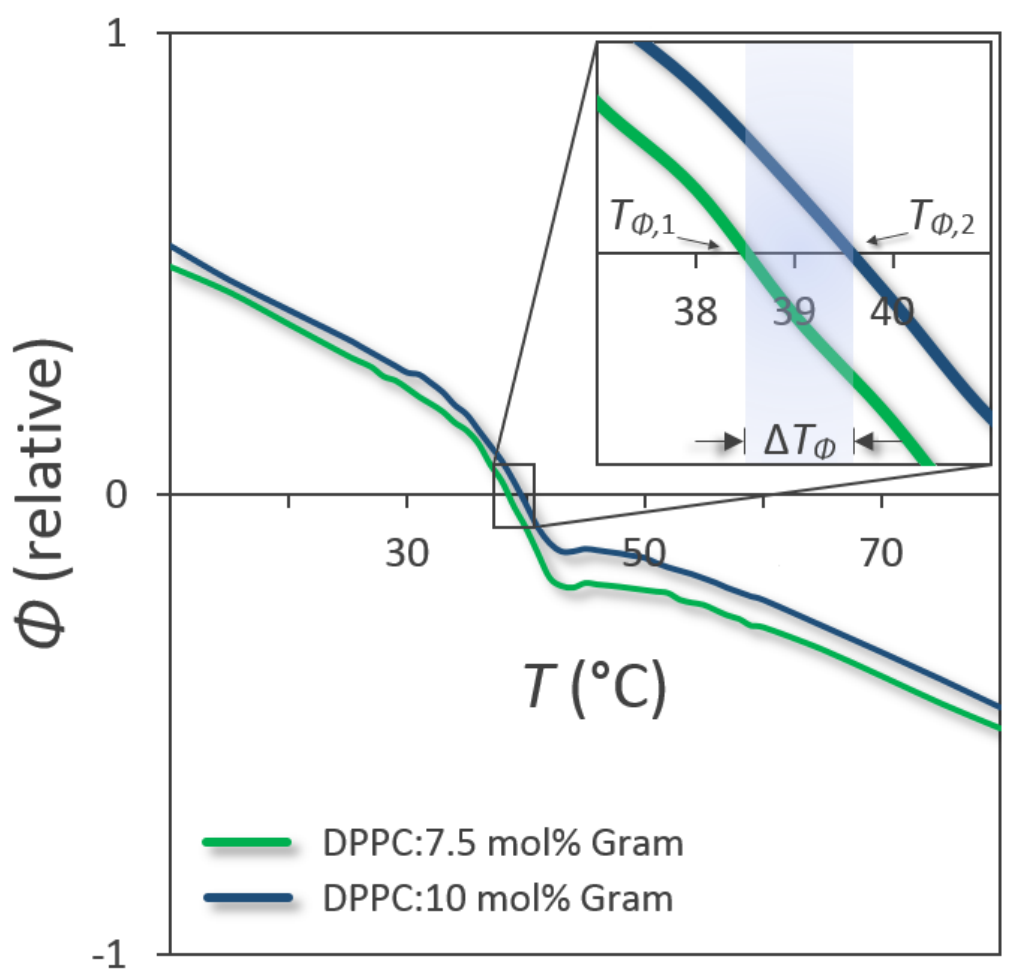

Figure 3-8 Relative values of $\Phi$ for DPPC vesicles $X_{g}=7.5$ (green) and $X_{g}=10 \%$ (blue). Inset: The allowable separation window for a mixed system composed of these two vesicle types is marked in blue, outlining the temperature range within which separation could be achieved due to opposite migrations of the vesicles. The relative values of $\Phi$ were calculated using vesicle compressibility data from the literature.

Alternatively, the separation window can be determined only based on the experimental measurements of $T_{\Phi}$ values, where no compressibility data exists for the systems of interest. The described opposite migrations in the microfluidic channel enables the collection of the enriched vesicles from separate outlets with high efficiencies (in this case exceeding 95\%). Figures 3.9a and b illustrate the 3D mapping of the fluorescent intensities of the blue and green channels across the width and a length of approximately $600 \mu \mathrm{m}$ of the channel at 37 and $39^{\circ} \mathrm{C}$, respectively. As evident from the figures, when the temperature has not exceeded the $T_{\Phi}$ of either of the two compositions, both vesicle compositions stay focused along the nodal plane in the center (Figure 3.9a).

Upon crossing the $T_{\Phi}$ of the $X_{g}=7.5 \%$ system, however, vesicles of this composition would migrate towards the antinodal planes at the walls leaving the $X_{g}=10 \%$ vesicles in the nodal plane (Figure 
3.9b). In determination of separation window for separation of cells, it should be noted that exposing cells to the temperatures higher than $43{ }^{\circ} \mathrm{C}$ is harmful and threaten the viability of the cells. ${ }^{209}$ However, there are a large number of cells/vesicles that reported to have lower transition temperatures, for which thermally-assisted acoustofluidic separation can be used safely. ${ }^{210,211}$

To verify the integrity of vesicles after separation, we reinjected the vesicles collected from the outlets into the device to examine their thermo-acoustofluidic behavior. We observed no significant difference in the $T_{\Phi}$ or the separation behavior of vesicles and concluded that essentially all of the vesicles survived the thermal modulation applied in the device.

\subsection{Analysis of Separation Performance Under Different Conditions}

The separation of $X_{g}=7.5 \%$ and $X_{g}=10 \%$ vesicle systems was in fact studied using different injected concentrations to examine the effect of starting vesicles population on the separation efficiency. In all separation experiments in this study, we quantify the efficiency of the separation in terms of purity which is defined as the ratio of the total number of the entity of interest to the total entities collected at the target outlet. Furthermore, the vesicles of the type with lower $T_{\Phi}$ were always labelled with green dye while the ones with the higher $T_{\Phi}$ were labelled with the blue dye.

The results obtained from the experiments conducted with different injected concentrations (Figure 3.9c) showed that lower starting concentrations expectedly result in higher purities at the outlets. This is, in part, due to the fact that at higher concentrations migrating vesicles would eventually saturate the available space at the walls, leading to their overflow to the center streamline or vice versa. Figure $3.9 \mathrm{c}$ clearly demonstrate that with increasing the injected vesicle concentrations the purities at both outlets drastically decreases to values lower than $60 \%$, indicating poorly effective separation. The other reason for the decrease is the steric hindrance from other vesicles. This is especially of significance at higher flow rates, when the residence time of vesicles in the channel are not sufficiently long. 

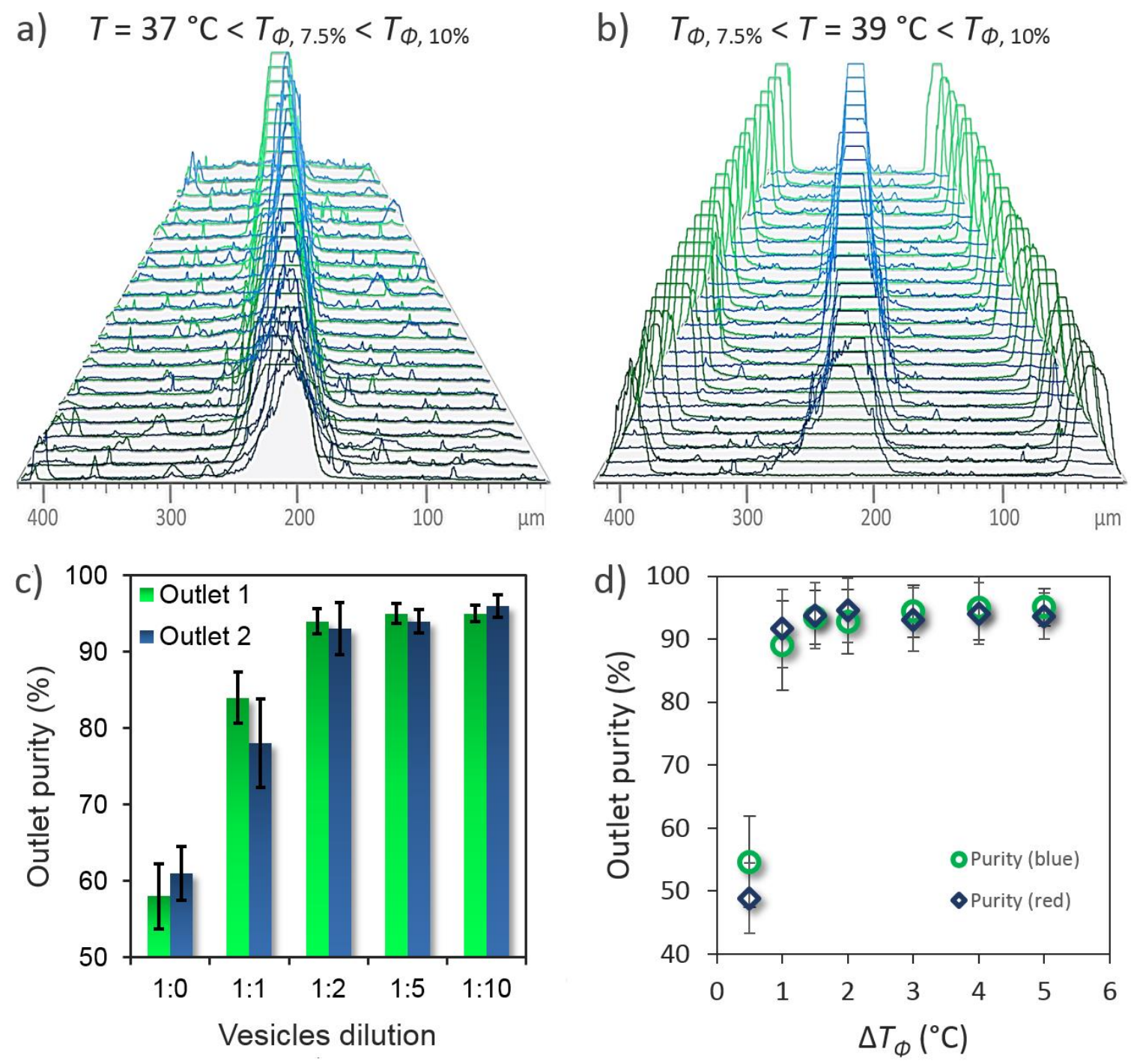

Figure 3-9 (a) and (b) 3D mapping of a separation event constructed using the fluorescent intensities of the blue and green channels across an area of approximately 400 by $600 \mu \mathrm{m}$ in the channel at respectively $37^{\circ} \mathrm{C}$, where the acoustic transducer is on but $T<T_{\Phi, 7.5 \%}<T_{\Phi, 10 \%}$, leading to the focusing of both systems in the center node, and at $39^{\circ} \mathrm{C}$, where the acoustic transducer is on and $T_{\Phi, 7.5 \%}<T<$ $T_{\Phi, 10 \%}$, causing the vesicles with $7.5 \mathrm{~mol} \%$ gramicidin to migrate towards the antinodes. (c) Outlet purity as a function of vesicles concentration in the channel (parts of the original suspension: parts of the diluent). Original concentration of the vesicles, 1:0, approximately corresponded to $10^{6}$ vesicles per $\mathrm{ml}$ of the suspension. (d) Outlet purity as a function of $\Delta T_{\Phi}$, i.e. the width of the allowable separation window, corresponding to the difference between the $T_{\Phi}$ S of constituent vesicle types of the binary mixture. (In all separation experiments, the system of lower $T_{\Phi}$ was labelled green and the one of higher $T_{\Phi}$ was blue. In addition, the flow rate in all experiments was $100 \mu \mathrm{l} \mathrm{hr} r^{-1}$, with $f_{1}$ and $V_{\mathrm{pp}}$ set to $1.4 \mathrm{MHz}$ and $50 \mathrm{~V}$, respectively.) The separation temperature was always selected at the middle temperature between $T_{\Phi 1}$ and $T_{\Phi 2}$. 
The initial composition of the vesicles before dilution (1:0) corresponded to $2 \%$ dissolved lipid in the organic solvent, yielding approximately $10^{6}$ vesicles per $\mathrm{ml}$ of the final suspension. The injection flow rate in all the separation experiments was set to $100 \mu \mathrm{l} \cdot \mathrm{hr}^{-1}$.

To further optimize the separations achieved by thermally-assisted acoustophoresis, separation experiments using multiple binary mixtures of vesicles with different compositions were performed to investigate the effect of $\Delta T_{\Phi}$, i.e. the width of the separation window, on the separation efficiency. The chosen binary mixtures of DMPC: gramicidin systems were $X_{g}=1 \%$ and $X_{g}=2.5 \%$ $\left(\Delta T_{\Phi} \approx 0.5\right), X_{g}=0 \%$ and $X_{g}=1 \%\left(\Delta T_{\Phi} \approx 1\right), X_{g}=0 \%$ and $X_{g}=2.5 \%\left(\Delta T_{\Phi} \approx 1.5\right), X_{g}=1 \%$ and $X_{g}=5 \%$ $\left(\Delta T_{\Phi} \approx 2\right), X_{g}=0 \%$ and $X_{g}=5 \%\left(\Delta T_{\Phi} \approx 3\right), X_{g}=2.5 \%$ and $X_{g}=7.5 \%\left(\Delta T_{\Phi} \approx 4\right)$, and $X_{g}=0 \%$ and $X_{g}=7.5 \%$ $\left(\Delta T_{\Phi} \approx 5\right)$. Figure 3.9d summarizes the results obtained from these experiments. In all experiments the channel temperature was maintained at the midpoint of the window range with the flow rate set to $100 \mu \mathrm{hr} r^{-1}$. As evident from the plot, when $\Delta T_{\Phi}$ is approximately $0.5^{\circ} \mathrm{C}$ wide, the purities at both outlets are close to $50 \%$, implying no effective separation in the channel. This is due to the very close values of the separation temperature compared to $T_{\Phi}$ of the vesicles migrating towards the wall $\left(T_{\text {separation }}=23.7^{\circ} \mathrm{C} v s . T_{\Phi, 1 \%}=23.5^{\circ} \mathrm{C}\right)$. Under such conditions, the $\Phi$ value remains close to zero and does not allow large enough acoustic radiation force to successfully drive the antinodal migration before reaching the channel split. When $\Delta T_{\Phi}$ is $1^{\circ} \mathrm{C}$ or wider, however, the purities at both outlets were improved to more than $92 \%$ for $\Delta T_{\Phi}=1^{\circ} \mathrm{C}$, and up to $97 \%$ in some cases for $\Delta T_{\Phi}$ wider than $2^{\circ} \mathrm{C}$. This improvement in separation efficiency is due to large enough $\Phi$ values assumed by vesicles after reaching temperatures that are significantly larger than the $T_{\Phi}$ to generate a large enough radiation force. In all cases, particularly with $\Delta T_{\Phi}<1{ }^{\circ} \mathrm{C}$, the purities can be further improved by intensifying the actuation parameters, such as increasing the power supplied or narrowing the channel, which can also reduce energy losses. Furthermore, slower flow rates, would extend the residence time of vesicles in the channel and allow them enough time to complete their migration towards their corresponding nodal or antinodal planes. 
Efficient acoustophoresis of EVs, particularly exosomes, due to the competing natures of the acoustic radiation force and acoustic streaming on the vesicles, has proved to be a challenging task..$^{212,213}$ Recently, however, a number of studies have presented improved designs that can indiscriminately, yet efficiently, trap EVs in a complex media. ${ }^{181-183}$ Furthermore, novel designs utilizing Fabry-Perot resonators have been recently shown to achieve reliable separations on the order of $100 \mathrm{~nm} .{ }^{214}$ Therefore, given the fact that the $\Delta T_{\Phi}$ of thermally-assisted acoustophoresis is independent of the size of the vesicles and merely harnesses the thermoelastic changes in the membrane, we expect that with more advanced resonator designs the current separation efficiencies and size limitation can be drastically improved.

\subsection{Conclusion}

In conclusion, this study demonstrates the separation of vesicles based on protein content using thermo-acoustophoresis. To our knowledge, this is the first report of such separation using a labelfree method. By relying on distinctive differences in vesicles thermophysical properties based on different peptide content, the separations of lipid vesicles containing gramicidin and alamethicin was accomplished. The separation efficiencies achieved in this study were as high as $97 \%$ without compromising the integrity of vesicles.

This label-free separation of vesicles based on membrane protein content opens new possibilities to the potential use of thermo-acoustophoresis in medical diagnoses that may involve microvesicles, exosomes, ectosomes, oncosomes, and other extracellular vesicles which may have real implications in various fields of medical research. 


\section{Addition of Melittin on Red Blood Cell Membrane}

\subsection{Introduction}

In this chapter, we examine the influence of membrane protein content (molar ratio) of particularly channel forming proteins, on the acoustophoretic behavior of sheep red blood cells. To investigate the effect of antimicrobial peptides on cells, we evaluated the thermo-acoustophoretic behavior of sheep red blood cells containing different amounts of melittin added to their membrane.

\subsection{Cell Preparation}

Sheep red blood cells (RBCs) (10\% suspension) were purchased from Fisher Scientific and diluted in Dulbecco's Phosphate Buffer Saline (DPBS) solution (from Gibco by life technologies). To introduce melittin to the cells, the peptide was first dissolved in DPBS and diluted to the desired concentration. Then, the diluted blood was added to the melittin solution kept at room temperature to reach the final dilution factor of 300 -fold for RBCs. Melittin containing cells were incubated at $37^{\circ} \mathrm{C}$ for 30 minutes before injection into the channel.

\subsection{The Effect of Melittin Concentration on Red Blood Cell Membrane}

Thermo-acoustophoretic behavior of red blood cells containing different amounts of melittin were investigated. As shown in Figures 4.1 to 4.4, a prompt nodal focusing was observed for RBCs containing 1,5 and $10 \mu \mathrm{M}$ melittin at $25^{\circ} \mathrm{C}$. This nodal focusing stays intact when increasing temperature to $37^{\circ} \mathrm{C}$. However, when melittin of $20 \mu \mathrm{M}$ concentration was added to RBCs, at both 25 and $37{ }^{\circ} \mathrm{C}$ the antinodal focusing was observed due to the negative sign of $\Phi$ at these temperatures. 

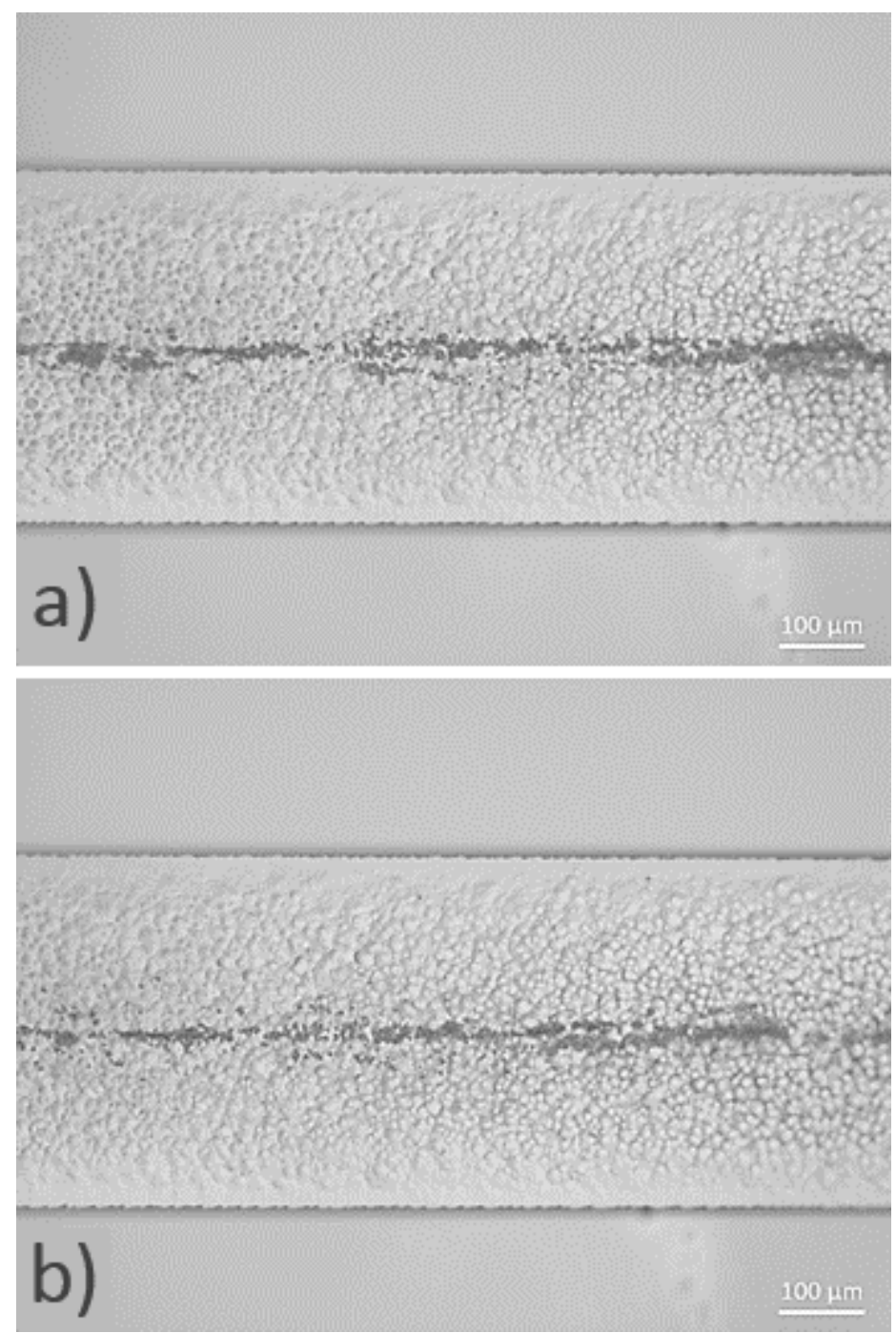

Figure 4-1 Optical microscope image of RBCs containing $1 \mu \mathrm{M}$ Melittin at (a) 25 and (b) $37^{\circ} \mathrm{C}$. 

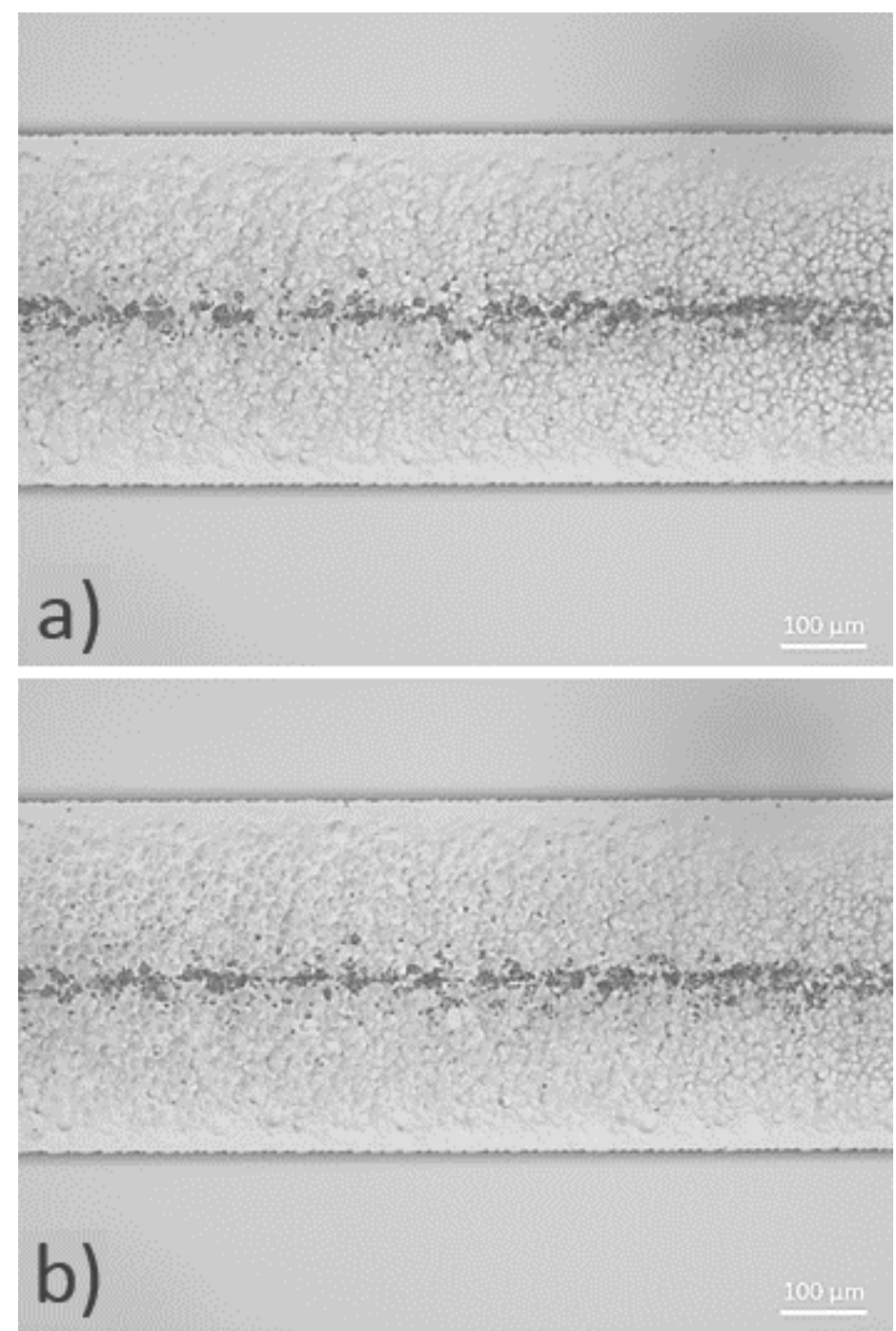

Figure 4-2 Optical microscope image of RBCs containing $5 \mu \mathrm{M}$ Melittin at (a) 25 and (b) $37^{\circ} \mathrm{C}$. 

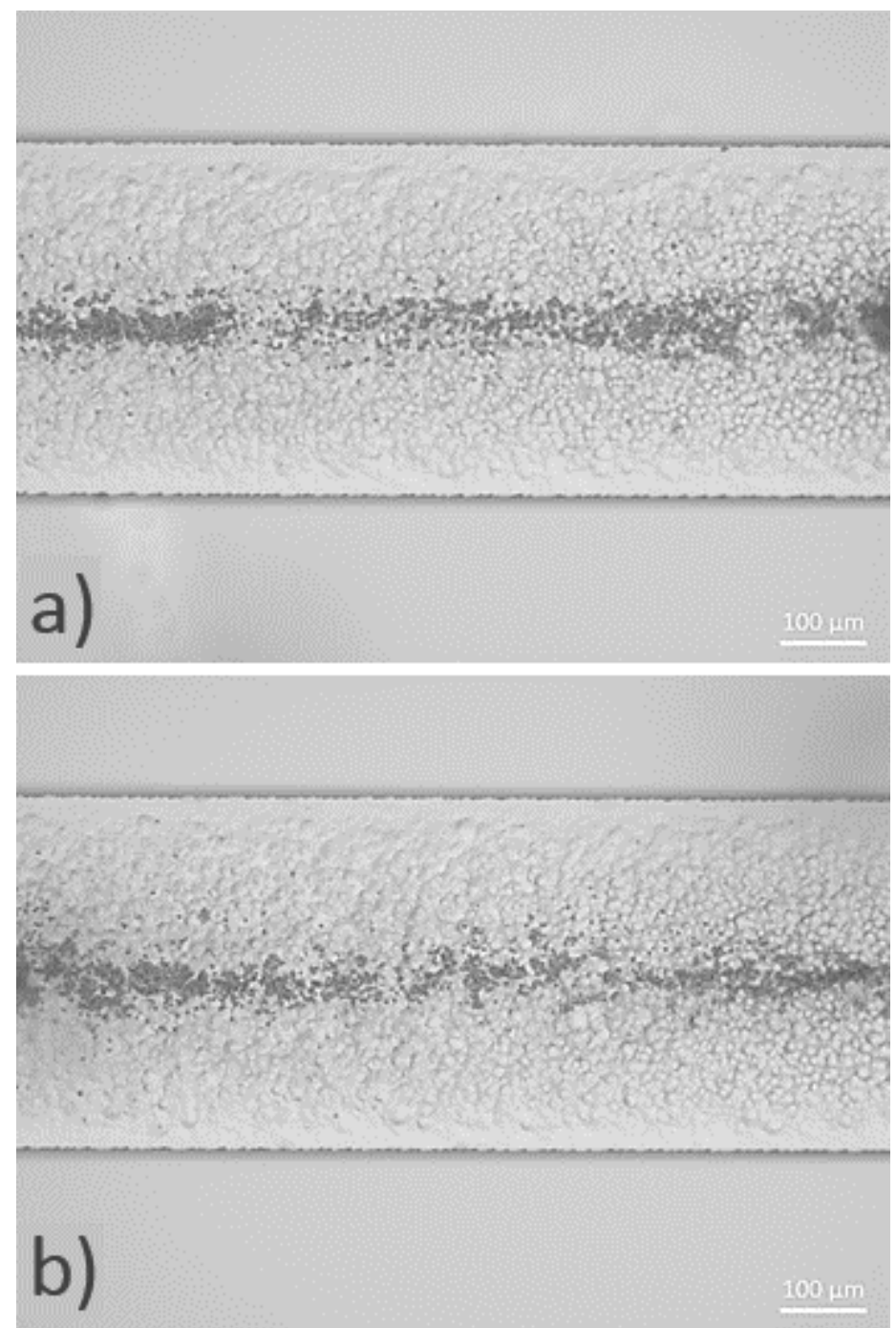

Figure 4-3 Optical microscope image of RBCs containing $10 \mu \mathrm{M}$ Melittin at (a) 25 and (b) $37^{\circ} \mathrm{C}$. 

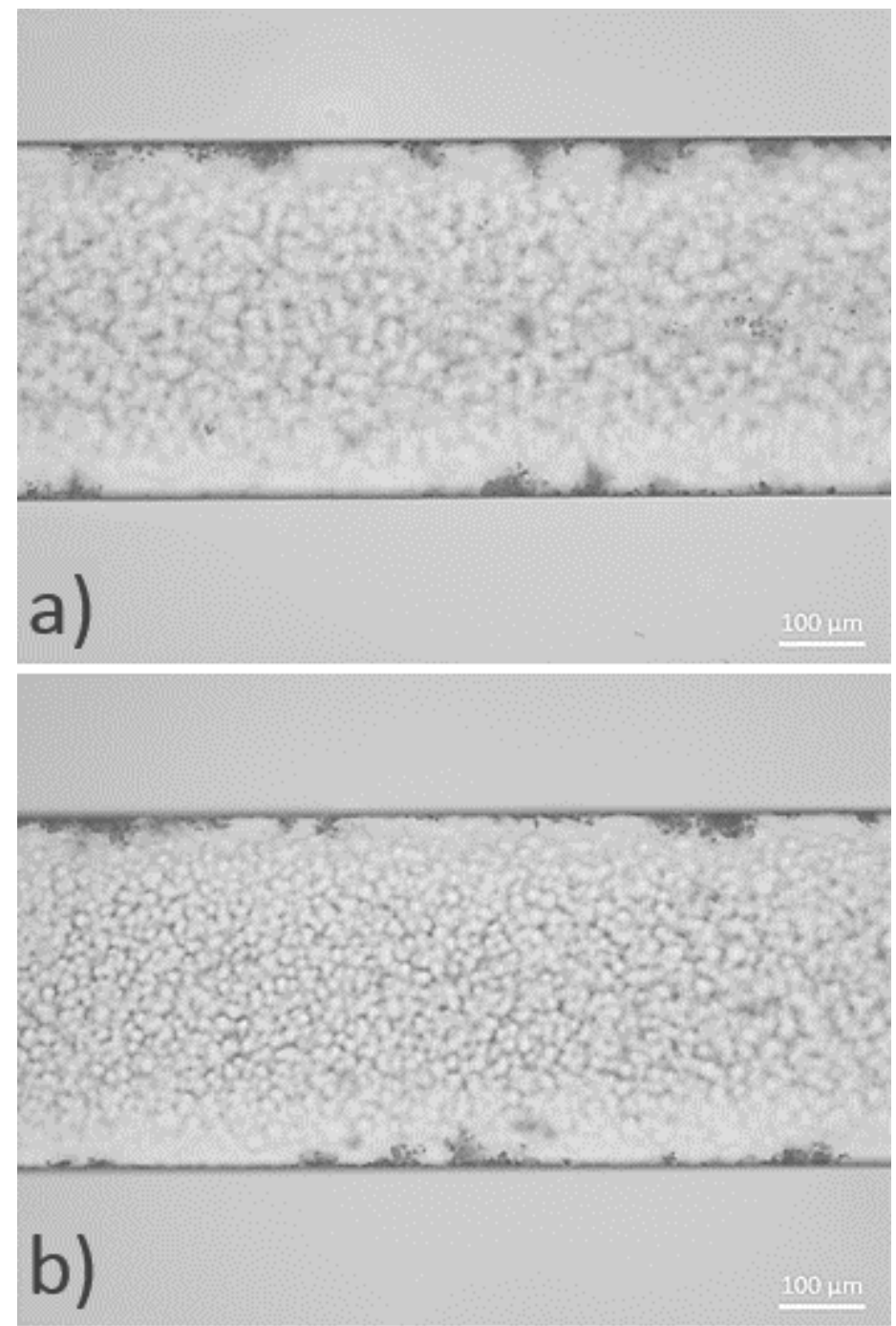

Figure 4-4 Optical microscope image of RBCs containing $20 \mu \mathrm{M}$ Melittin at (a) 25 and (b) $37^{\circ} \mathrm{C}$.

\subsection{Thermo-Acoustofluidic Behavior RBCs Containing Melittin}

At $37{ }^{\circ} \mathrm{C}$ and in the absence of melittin, RBCs exhibit a positive $\Phi$, leading to their swift nodal focusing. Figure 4-5a shows a micrograph taken from these cells at the center of the channel when transducer was set at the first harmonic frequency. This behavior was expected as cells in their native state are known to show a positive $\Phi$ under physiological conditions. In this regard, RBCs remained focused at the node when the temperature was increased to $42{ }^{\circ} \mathrm{C}$. However, upon 
addition of melittin following the outlined procedure, a negative $\Phi$ was observed at $37{ }^{\circ} \mathrm{C}$ and an anti-nodal focus-ing proceeded when the channel was actuated at the first harmonic frequency (Figure 4-5b).

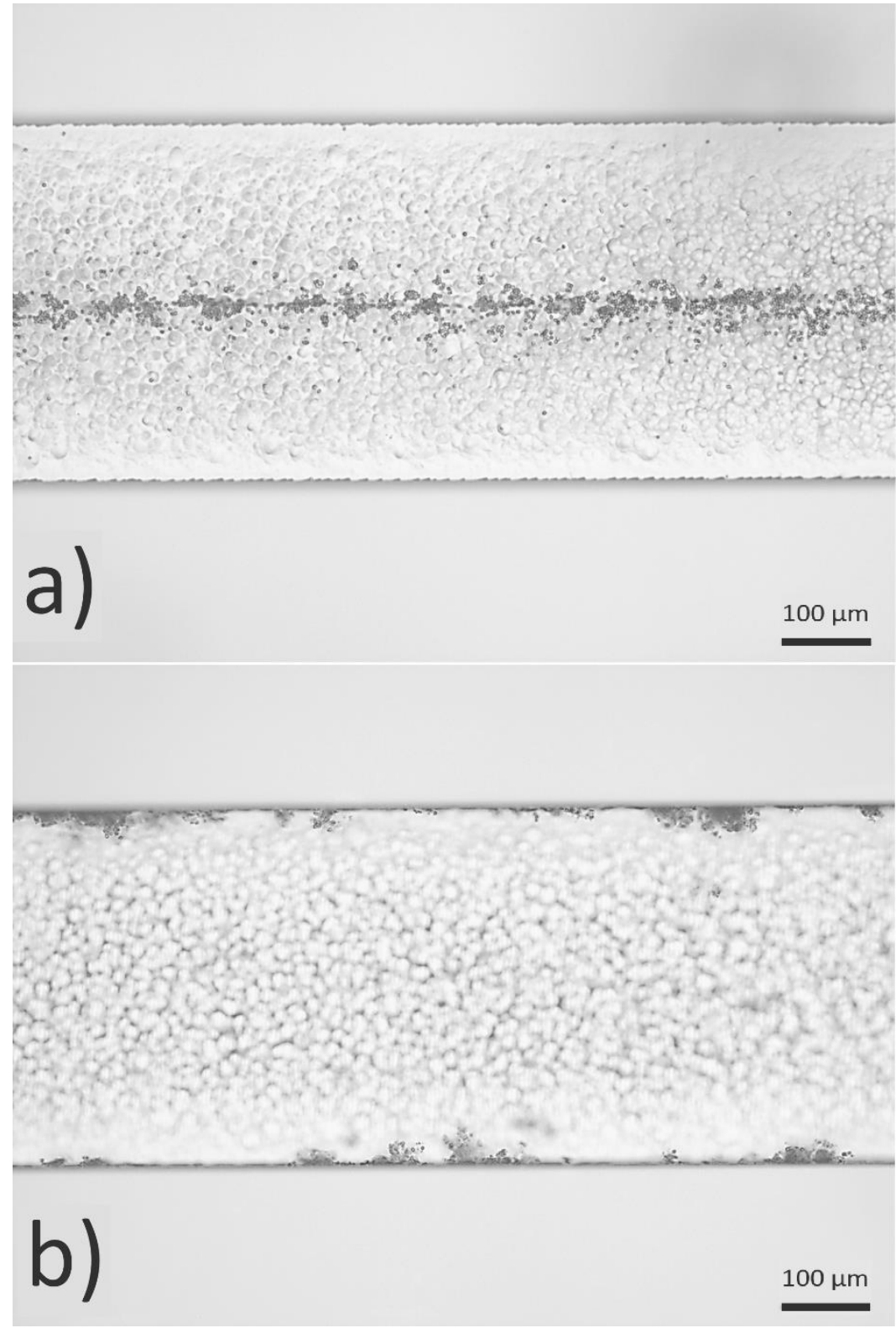

Figure 4-5 Optical micrographs of red blood cells response to acoustic actuation at the first harmonic frequency at $37^{\circ} \mathrm{C}$; (a) when no melittin is present in the membrane, a nodal focusing occurs which is stable upon increasing temperature up to $42^{\circ} \mathrm{C}$, and (b) when $20 \mu \mathrm{M}$ melittin was added to the system; an antinodal focusing is observed even at room temperature, implying a decrease in $\Phi$ to negative values. 
The same behavior was observed even when the channel was actuated at room temperature, indicating the significant effect of melittin at minute concentrations on the mechanical properties of the RBCs membrane, which is manifested through a switch in the sign of $\Phi$. In RBCs, similar to PC vesicles, melittin disrupted the membrane upon its introduction. ${ }^{215-217}$ Melittin 'monomers' first started partitioning into the membrane ${ }^{218}$, which is followed by the aggregation of 'tetramers' that creates 'toroidal' or 'barrel stave' pore structures and causes disorder in the membrane. ${ }^{215-218}$ The formation of these stable pores hinders membrane fluctuations and alters its deformability. ${ }^{219}$ The addition of melittin to RBCs also induced some morphological changes from discocytes to echinocytes (burr cells) and spherocytes. ${ }^{219}$ To our knowledge, this is the first report of a peptideinduced acoustic contrast modification in cells. 


\section{Separation of Vesicles from Cancer Cells Using Thermally-assisted Acoustophoresis}

There exists a growing interest in the study of extracellular vesicles (EVs) as potential biomarkers for health and various types of diseases. ${ }^{166-171,220,221}$ EVs are secreted from cells to act as intercellular communicators, carry waste cargoes and perform many other specialized functions. 20,21,222 Hence, they contain valuable information about their parent cells' biology. ${ }^{223-226}$ In this regard, the compositional analysis of EVs secreted from cancer cells has been shown to provide information on the status of the disease or the treatment effectiveness. ${ }^{19,22-24} \mathrm{~A}$ case in point is the vesicles shed from breast cancer cells which were shown to differ from the ones originating from normal cells in terms of carrying signaling information about the tumor to surrounding tissue. ${ }^{227}$ This type of signaling has been suggested to be responsible for either promoting tumorigenesis or tuning immune response. ${ }^{21,222}$ Although EVs are abundant in circulating biological media, their studies are restricted by the resolution of isolation techniques. ${ }^{19,22-24}$ Most of the available techniques such as differential centrifugation and ultrafiltration function based on size differences between vesicles, and are therefore incapable of discriminating between vesicles based on their origin indicators like composition. ${ }^{19,22-24,173}$ In addition, the small size of EVs makes their differentiation from other components in the media challenging. ${ }^{228}$

Microscopy techniques such as electron microscopy are likewise incapable of differentiating EVs, as different EVs overlap in their size and morphology. ${ }^{26}$ Therefore, a reliable method to separate EVs based on their constituents that is relatively inexpensive and could provide results in a reasonable time is of great significance in biomarker discovery.

The application of acoustophoresis as a gentle and rapid lab-on-a-chip method in differentiating circulating EVs from cells has been traditionally considered to be challenging as a result of the small size of the EVs. More recently, however, modified acoustophoretic devices were shown to be capable of separating particles with submicron size even on the order of $100 \mathrm{~nm} .{ }^{214}$ 


\subsection{Cholesterol Containing Vesicles}

The membrane of biological vesicles is composed of many different lipids and embedded proteins and carbohydrates. Each lipid in the membrane is unique in terms of the head group or constituent fatty acids which determine the differences in the physical properties of lipids. A frequently available lipid in biological membranes is cholesterol. ${ }^{186}$ Although this lipid cannot form a bilayer on its own its presence plays an influential role in the membrane stiffness. Therefore, cholesterol can be used in vesicles to artificially regulate the membrane stiffness. ${ }^{186}$

The vesicles in this study were prepared using a phosphatidylcholine (PC) with saturated fatty acyl chains-1,2-dimyristoyl-sn-glycero-3-phosphocholine (DMPC) - and cholesterol which were respectively purchased from Avanti and Sigma-Aldrich and were used without further purification. The preparation of vesicles was based on the already described method in section 2-3 comprising of first dissolving the desired lipid in the water-miscible solvent tetrahydrofuran, followed by the addition of a fluorescent dye, namely Nile red (9-diethylamino-5-benzo[ $\alpha]$ phenoxazinone) and Laurdan (Acros Organics). In the last step, distilled water was added dropwise to the solution, causing lipid bilayers to form vesicles.

\subsection{Breast Cancer Cells}

MCF-7 epithelial breast cancer cells, purchased from ATCC, were cultured at $37{ }^{\circ} \mathrm{C}$ and $5 \% \mathrm{CO} 2$ in Eagle's Minimum Essential Medium (EMEM, ATCC) supplemented with 10\% Fetal bovine serum (FBS, Sigma Aldrich), 1\% Penicillin- Streptomycin (Fisher Scientific), and $0.01 \mathrm{mg} / \mathrm{mL}$ human recombinant insulin (Sigma Aldrich). Cells were sub-cultured upon reaching 80\% confluency by removing media, rinsing with Dulbecco's Phosphate-Buffered Saline (DPBS, Sigma Aldrich), and incubating in Trypsin-EDTA (Sigma Aldrich) for 5 minutes. In the next step, suspended cells and $6 \mathrm{~mL}$ of growth medium were transferred to a centrifuge tube and centrifuged at $125 \mathrm{~g}$ for 5 minutes. Supernatant was removed and cells were resuspended in fresh growth 
medium. Cells were stained prior to subculture protocol by removing the growth medium and incubating for approximately 15 minutes at $37{ }^{\circ} \mathrm{C}$ in $0.5 \mu \mathrm{M}$ Vybrant CFDA SE Cell Tracer Kit (ThermoFisher, V12883). The stain solution was subsequently removed, fresh growth medium was added, and cells were incubated for an additional 30 minutes.

\subsection{Thermo-Acoustophoretic Behavior of Vesicles in a Media Containing Cancer Cells}

Large unilamellar and multilamellar vesicles, $600 \mathrm{~nm}$ to $1 \mu \mathrm{m}$ in diameter, with two different cholesterol contents were prepared to mimic EVs of different compositions found in complex media containing other components like cells. Cholesterol was chosen as a composition modifier for two main reasons; first, it has been shown that in various types of cancers the feedback mechanisms by which the cholesterol content of the cells is regulated are lost, leading to up- or down-regulation of cholesterol synthesis in the cells. ${ }^{229}$ In the case of breast cancer, specifically, the cholesterol content of the cells tends to increase as the cell assumes a phenotypic state corresponding to the initiation of cancer. ${ }^{230}$ Secondly, small amounts of cholesterol can bring about considerable changes in the thermo-acoustophoretic properties of vesicles. ${ }^{129,186}$ Therefore, given the fact that the composition and consequently the role of EVs is largely determined by their parent cell's composition, the feasibility of separating submicron vesicles differing in their composition in a media containing various components could be of great significance in many biomedical applications. The experiments in this study were accordingly aimed at achieving the same goal by isolating one and two types of vesicles from a medium spiked with cancer cells. The compositions of the vesicles used were 90:10 DMPC:CHOL and 80:20 DMPC:CHOL. The human adenocarcinoma breast cell line MCF-7, usually considered to be a good mimic of circulating breast cancer cells, was used in the mixture to model the circulating tumor cells (CTCs). The thermo-acoustophoretic behavior of each vesicle system was studied prior to the separation experiment. For doing so, each vesicle system was first injected at $20{ }^{\circ} \mathrm{C}$ into a single-stage channel with two outlets. Following that, the piezoelectric transducer was switched on at the first harmonic frequency of the channel which as 
expected resulted in a prompt movement of the vesicles towards the center node of the channel. Upon heating the channel at $1{ }^{\circ} \mathrm{C} / \mathrm{min}$, a node-to-antinode transition was observed at the acoustic contrast temperature $\left(\mathrm{T}_{\Phi}\right)$, as observed in our previous studies. These temperatures which are unique for each system were measured to be 24.2 and $29.2{ }^{\circ} \mathrm{C}$ for the $90: 10$ and $80: 20$ DMPC:CHOL systems, respectively. The $\mathrm{T}_{\Phi}$ of the MCF-7 cells was not observed to occur in the 20 to $35{ }^{\circ} \mathrm{C}$ temperature range, making the separation window big enough to have reliable separation between the vesicles and cells.

After establishing the temperature window for each system, for the separation tests consisting of one type of DMPC:CHOL vesicles and MCF-7 cells, the temperature of the channel was set to a temperature higher than the $\mathrm{T}_{\Phi}$ of the respective vesicle system and was kept constant throughout the experiment. Figure 5.1(a) shows an optical micrograph of the randomly dispersed 80:20 DMPC:CHOL vesicles in the single-stage channel at $20{ }^{\circ} \mathrm{C}$. Upon increasing the temperature to higher than $29.2{ }^{\circ} \mathrm{C}$, vesicles undergo a thermotropic transition and migrate to the walls of the channel (antinodal migration) while MCF-7 cells remain at the center of the channel (pressure nodes). The separation here is realized through the fact that the selected channel temperature (Tseparation) is chosen such that it is greater than the $\mathrm{T}_{\Phi}$ of 80:20 DMPC:CHOL vesicles and is lower than the $\mathrm{T}_{\Phi}$ of the cells. Figure 5.1 (b) shows the same system after the temperature of the channel has reached $31{ }^{\circ} \mathrm{C}$ while the transducer is on.

After the separation, the average purity of the collected vesicles at the target outlet was calculated to be exceeding 94\% For both 80:20 DMPC:CHOL/MCF-7 and 90:10 DMPC:CHOL/MCF-7 systems. Figure 5.3 shows the outlet purity of the experiments consisting of DMPC:CHOL vesicles and MCF-7 cells. 


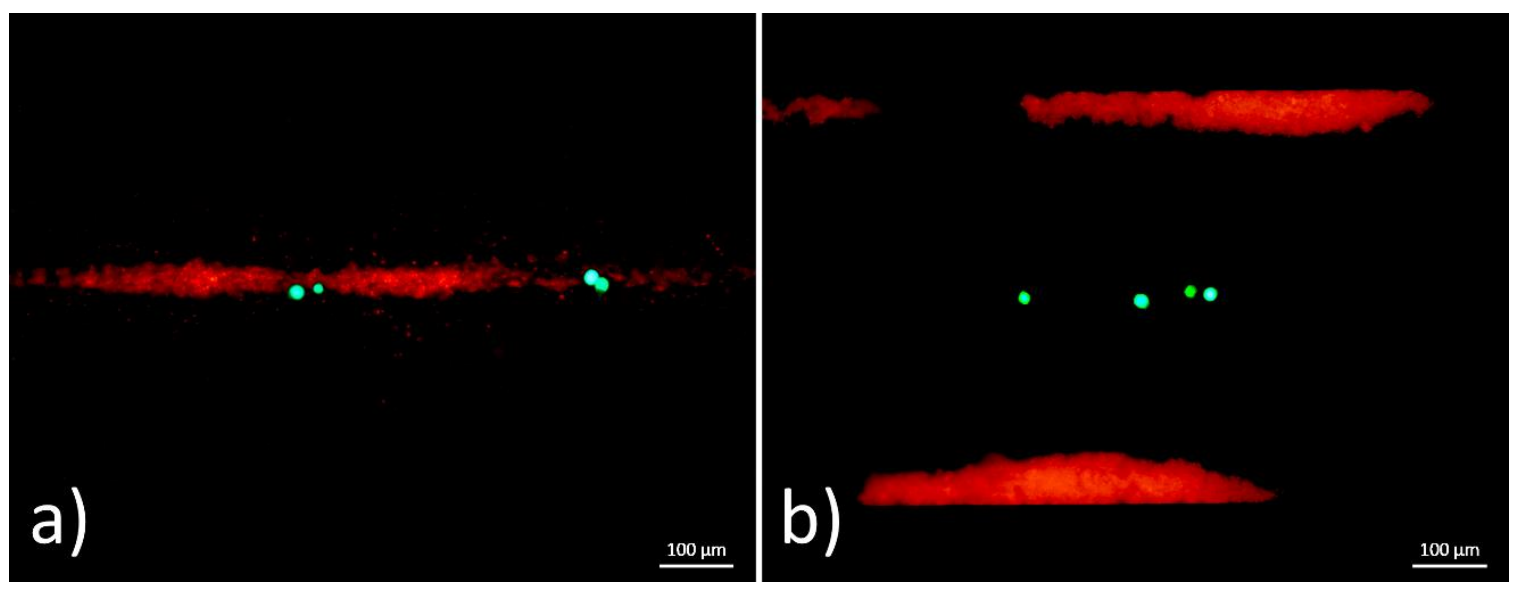

Figure 5-1 a) Focusing of a system consisting of MCF-7 cells (green) and 80:20 DMPC:CHOL vesicles (red) at $20{ }^{\circ} \mathrm{C}$ when the acoustic transducer is on. b) The same system after the temperature of the channel has reached $31{ }^{\circ} \mathrm{C}$.

In a more complex system, where the medium contains two different types of vesicles-90:10 DMPC:CHOL and 80:20 DMPC:CHOL—and MCF-7 cells, the separation temperature should be set within the temperature window of the two corresponding system of interest to achieve a separation between those two systems. Depending on the objective of the experiment and the relative $\mathrm{T}_{\Phi}$ values of the respective systems, usually measured in advance, one could use a singlestage or two-stage separation device for the thermally-assisted acoustophoretic experiments. Since the $\mathrm{T}_{\Phi}$ of the MCF-7 cells was not observed to occur in the 20 to $35^{\circ} \mathrm{C}$ temperature range, isolation of either type of the vesicles would require a two-stage device, where the first stage temperature (Tseparation,1) is greater than the smallest $\mathrm{T}_{\Phi}\left(24.2{ }^{\circ} \mathrm{C}\right.$ for $90: 10$ system) and the second stage temperature (Tseparation,2) is greater than the $\mathrm{T}_{\Phi}$ of the second vesicle type $\left(29.2{ }^{\circ} \mathrm{C}\right.$ for $80: 20$ system). In all experiments, the temperature for each stage was fixed at the assigned separation temperature $\left(\mathrm{T}_{\text {separation }}\right)$ while the mixture was injected into the channel. The vesicle-cell mixtures dispersed randomly in the channel before switching on the acoustic transducer. Upon turning on the acoustic transducer at $27{ }^{\circ} \mathrm{C}$, since the separation temperature is greater than the $\mathrm{T}_{\Phi}$ of $90: 10$ DMPC:CHOL vesicles (blue) and is yet less than the $\mathrm{T}_{\Phi}$ of 80:20 DMPC:Chol vesicles (red) and 
cells (green), an antinodal migration for the 90:10 system and a nodal migration of 80:20 system and cells were observed, leading to the separation of the first type of vesicles through the first pair of side channels (Figure 5.2 (a)). In the second stage, where the temperature is set to be $31^{\circ} \mathrm{C}$, since the $\mathrm{T}_{\text {separation }}$ is now greater than the $\mathrm{T}_{\Phi}$ of 80:20 DMPC:CHOL vesicles, these vesicles are also driven towards the antinode, leaving behind the cells in the center. Therefore, the second type of vesicles can be collected from the next pair of side channels located at the second stage of the device. It is worth mentioning that only a localized temperature rise after the first series of side channels would lead to the successful separation 80:20 DMPC:CHOL vesicles from 90:10 DMPC:CHOL vesicles. Figure 5.3 part 3 shows the outlet purity of the cells and vesicles collected after the experiments consisting of two types of DMPC:CHOL vesicles and MCF-7 cells, indicating a reliable separation for all the components. In all experiments, the actuation voltage and mixing ratio of the vesicles were constant.
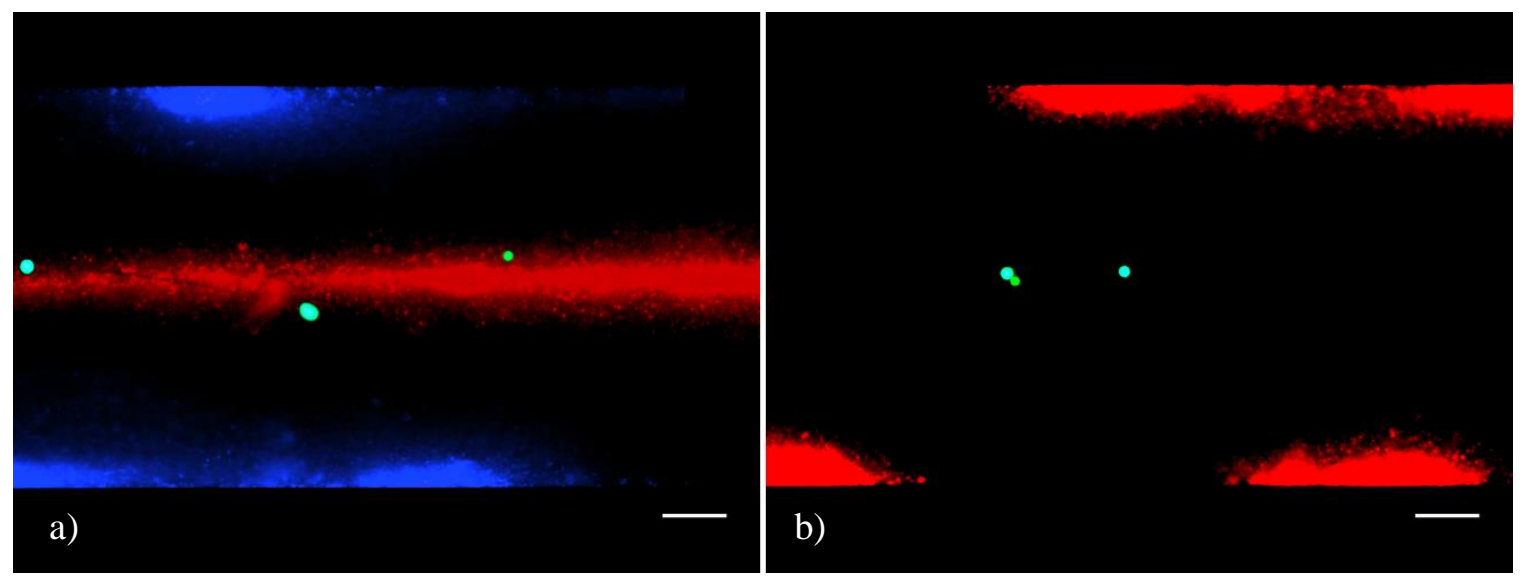

Figure 5-2 a) Thermo-acoustophoretic behavior of a system consisting of MCF-7 cells (green), 90:10 DMPC:CHOL (blue) and 80:20 DMPC:CHOL vesicles (red) at $27^{\circ} \mathrm{C}$ when the acoustic transducer is on. b) The same system at the second stage of the channel where the temperature of is kept at $31{ }^{\circ} \mathrm{C}$. 


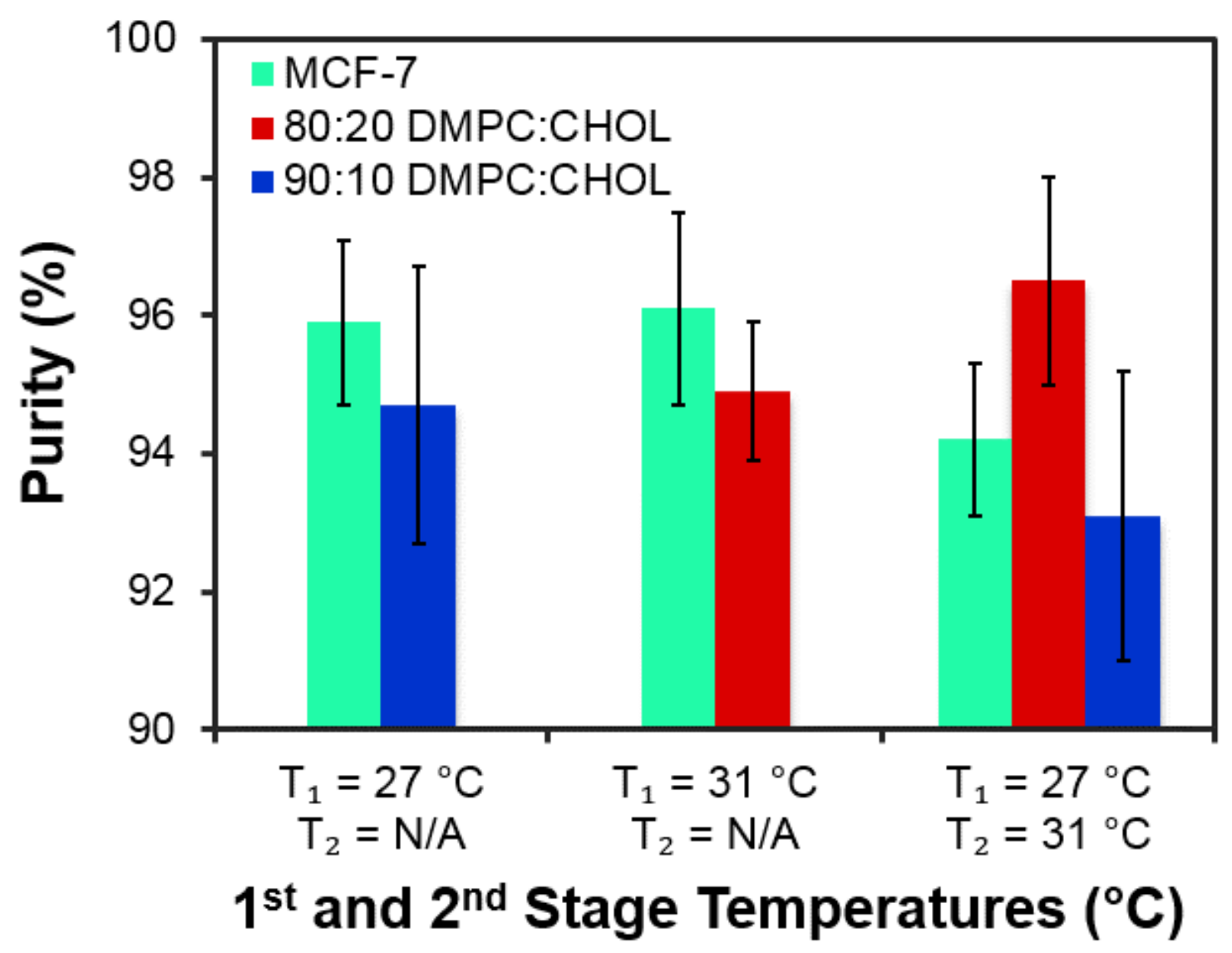

Figure 5-3 Purity of the vesicles and cells collected from the single-stage and two-stage devices. The first two series of clustered columns on the left present the data obtained from experiments in the single-stage device with 90:10 DMPC:CHOL/MCF-7 and 80:20 DMPC:CHOL/MCF-7 systems. The right-hand side columns show the purity data obtained from the separation study performed in the two-stage device with the three-component system. $T_{1}$ indicates the temperature of the first Peltier element ( $1^{\text {st }}$ stage of the channel) and $T_{2}$ indicates the temperature of the second Peltier element $\left(2^{\text {nd }}\right.$ stage). Since the single-stage device was equipped with only one Peltier element, only one temperature is reported for the experiments performed in that device.

\subsection{Conclusion}

In these experiments, thermally-assisted acoustophoresis was successfully employed to demonstrate the isolation of submicron vesicles with variable cholesterol contents from a media containing cancer cells. Further to the high separation efficiencies (over 93\%) achieved for the vesicles, the MCF-7 cells themselves which were acting as a mimic for the circulating breast tumor cells were also isolated with high efficiency (over 94\%). We believe this is the first report of an 
ultrasonically-actuated device capable of separating vesicles based on their composition from a medium containing other components like cells. We envision that the thermo-acoustophoretic principles applied in this study may be well suited as a separation criteria to be used with other ultrasonically-driven devices made for sorting extremely small extracellular vesicles based on their origin and/or function in complex biological media. 


\section{Summary}

This dissertation presents the capability of a thermally-assisted acoustophoresis technique for the separation of membrane-bound systems such as vesicles based on their proteome and lipidome content. The thermally-dependent acoustic properties of membrane bound systems constituting of unary and binary lipid compositions were examined in an ultrasonic standing wave actuated in the first harmonic frequency mode. Then three different transmembrane proteins were introduced to the membrane of such systems and the effect of protein content on their thermos-acoustofluidic behavior was investigated. We have demonstrated that synthetic and natural membrane bound systems such as vesicles and cells, when placed in an ultrasonic standing wave, exhibit a nodal focusing meaning that they typically possess a positive value of acoustic contrast factor $(\phi)$. Upon changing the temperature, a nodal to antinodal migration of vesicles in a standing wave happens due to a reversible thermotropic changes in the lipid bilayer of vesicles rendering a $\phi$ sign change. $T_{\phi}$ is the temperature at which a switch in the acoustic focusing of the vesicles from the node to antinodes happen. Investigation into the behavior of lipid bilayers under thermo-acoustophoresis revealed a distinct temperature for each vesicle system based on their lipid composition and protein content. The specific conclusions on the effect of proteome and lipidome on the behavior of membrane bound systems in the thermally-assisted acoustophoresis and the potential of this method to be used in bioanalytical applications are listed below:

- Using the unique acoustic contrast temperature $\left(T_{\phi}\right)$ of each vesicle system a separation of two vesicle systems was developed at $T_{\phi 1}<T_{\text {separation }}<T_{\phi 2}$. Under this separation temperature condition, vesicles migrated in opposite directions, towards the central region or the wall regions of the channel.

- Thermally-assisted acoustophoresis was successfully employed to demonstrate the effect of protein content on the acoustic contrast temperature of lipid membrane bound systems. 
- Transmembrane proteins, depending on their type and content in the lipid membrane, either order the structure of the bilayer or disorder it leading to an increase or decrease in the acoustic contrast temperature.

- This study demonstrates the separation of vesicles based on protein content using thermoacoustophoresis. To our knowledge, this is the first report of such separation using a label-free method.

- By relying on distinctive differences in vesicle thermophysical properties based on different peptide content, the separations of lipid vesicles containing gramicidin, alamethicin and melittin was accomplished.

- The separation efficiencies achieved in this study were as high as $97 \%$ without compromising the integrity of the vesicles.

- This label-free separation of vesicles based on membrane protein content open new possibilities to the potential use of thermo-acoustophoresis in medical diagnoses that may involve microvesicles, exosomes, ectosomes, oncosomes, and other extracellular vesicles which may have real implications in various fields of medical research.

- Thermally-assisted acoustophoresis was successfully employed to demonstrate the isolation of submicron vesicles with variable cholesterol contents from a media containing cancer cells.

- High separation efficiencies (over 93\%) were achieved for the vesicles containing different amounts of cholesterol.

- Further to the high separation efficiencies achieved for the vesicles, the MCF-7 epithelial breast cancer cells themselves, which were acting as a mimic for the circulating breast tumor cells, were also isolated with high efficiency (over 94\%).

- The successful separation of vesicles based on their composition from a medium containing other components like cells using an ultrasonically-actuated device was achieved for the first time. 
- We envision that the thermo-acoustophoretic principles applied in this study may be well suited as a separation criteria to be used with other ultrasonically-driven devices made for sorting extremely small extracellular vesicles based on their origin and/or function in complex biological media. 
References

1. Jacobs, M. H. Early Osmotic History of the Plasma Membrane. Circulation XXVI, (1962).

2. Kleinzeller, A. in Membrane Permeability (eds. Deamer, D. W., Kleinzeller, A. \& Fambrough, D. M. B. T.-C. T. in M.) 48, 1-22 (Academic Press, 1999).

3. Langmuir, I. THE CONSTITUTION AND FUNDAMENTAL PROPERTIES OF SOLIDS AND LIQUIDS. II. LIQUIDS.1. J. Am. Chem. Soc. 39, 1848-1906 (1917).

4. Gao, J. \& Wang, H. History and Traditional Techniques of Studying the Structure of Cell Membranes. 21-44 (2018).

5. Lombard, J. Once upon a time the cell membranes : 175 years of cell boundary research. Biol. Direct 1-35 (2014). doi:10.1186/s13062-014-0032-7

6. Nicolson, G. Update of the 1972 Singer-Nicolson Fluid-Mosaic Model of Membrane Structure. Discoveries 1, (2013).

7. Heimburg, T. Thermal Biophysics of Membranes. (WILEY-VCH Verlag GmbH \& Co. $\mathrm{KGaA}, 2007)$.

8. Mouritsen, O. L. E. G. \& Bloom, M. MATTRESS MODEL OF LIPID-PROTEIN INTERACTIONS IN MEMBRANES. Biophys. J. 46, 141-153 (1982).

9. Heimburg, T. Thermal biophysics of mambranes. (WILEY-VCH). doi:10.15713/ins.mmj.3

10. Kaiser, H. et al. Lateral sorting in model membranes by cholesterol- mediated hydrophobic matching. PNAS 108, 16628-16633 (2011).

11. Bouvrais, H. Bending Rigidities of Lipid Bilayers: Their Determination and Main Inputs in Biophysical Studies. Advances in planar lipid bilayers and liposomes 15, (Elsevier Ltd, 2012).

12. Cell Lipids. (Academic Press, Inc., 1994).

13. Marsh, D. Handbook of Lipid Bilayers. (CRC Press, Taylor \& Francis Group, LLC, 2013).

14. Lichtenberg, D. \& Barenholz, Y. in Methods of Biochemical Analysis, Volume 33 (ed. Glick, D.) 337-462 (John Wiley \& Sons, Inc., 1988).

15. Phillips, R., Kondev, J., Theriot, J. \& Garcia, H. G. Physical Biology of the Cell. (Garland Science, Taylor \& Francis Group, LLC, 2013).

16. M, E., M, D., Eeman, M. \& Deleu, M. From biological membranes to biomimetic model membranes. Focus (Madison). 14, 719-736 (2010).

17. Cheng, Y. et al. Single ion channel sensitivity in suspended bilayers on micromachined supports. Langmuir 17, 1240-1242 (2001).

18. Sezgin, E., Levental, I., Mayor, S. \& Eggeling, C. The mystery of membrane organization: composition, regulation and roles of lipid rafts. Nat Rev Mol Cell Biol advance on, (2017).

19. Raposo, G. \& Stoorvogel, W. Extracellular vesicles: Exosomes, microvesicles, and friends. J. Cell Biol. 200, 373-383 (2013). 
20. Momen-Heravi, F. et al. Current methods for the isolation of extracellular vesicles. Biol. Chem. 394, 1253-1262 (2013).

21. Koniusz, S. et al. Extracellular Vesicles in Physiology, Pathology, and Therapy of the Immune and Central Nervous System, with Focus on Extracellular Vesicles Derived from Mesenchymal Stem Cells as Therapeutic Tools. Front. Cell. Neurosci. 10, (2016).

22. Yáñez-Mó, M. et al. Biological properties of extracellular vesicles and their physiological functions. J. Extracell. Vesicles 4, 27066 (2015).

23. Gould, S. J. \& Raposo, G. As we wait: Coping with an imperfect nomenclature for extracellular vesicles. J. Extracell. Vesicles 2, 20389 (2013).

24. Borges, F., Reis, L. \& Schor, N. Extracellular Vesicles: Structure, Function and Potential Clinical Uses in Renal Diseases. Brazilian J. Med. Biol. Res. 46, 824-830 (2013).

25. Balaj, L. et al. Tumour microvesicles contain retrotransposon elements and amplified oncogene sequences. Nat. Commun. 2, 180 (2011).

26. van der Pol, E., Boing, A. N., Harrison, P., Sturk, A. \& Nieuwland, R. Classification, Functions, and Clinical Relevance of Extracellular Vesicles. Pharmacol. Rev. 64, 676-705 (2012).

27. Burden, H. P. Prostasomes--their effects on human male reproduction and fertility. Hum. Reprod. Update 12, 283-292 (2006).

28. Ronquist, G. \& Nilsson, B. O. The Janus-faced nature of prostasomes: their pluripotency favours the normal reproductive process and malignant prostate growth. Prostate Cancer Prostatic Dis 7, 21-31 (2004).

29. Al-Nedawi, K. et al. Intercellular transfer of the oncogenic receptor EGFRvIII by microvesicles derived from tumour cells. Nat. Cell Biol. 10, 619-624 (2008).

30. Meehan, B., Rak, J. \& Vizio, D. Di. Oncosomes - large and small: what are they, where they came from. J. Extracell. Vesicles 1, 4-5 (2016).

31. Lee, T. H. et al. Microvesicles as mediators of intercellular communication in cancer-the emerging science of cellular 'debris'. Semin. Immunopathol. 1-13 (2011). doi:10.1007/s00281-011-0250-3

32. Nakano, I., Garnier, D., Minata, M. \& Rak, J. Extracellular vesicles in the biology of brain tumour stem cells - Implications for inter-cellular communication, therapy and biomarker development. Semin. Cell Dev. Biol. 40, 17-26 (2015).

33. Kolusheva, S., Shahal, T. \& Jelinek, R. Peptide-membrane interactions studied by a new phospholipid/polydiacetylene colorimetric vesicle assay. Biochemistry 39, 15851-15859 (2000).

34. Kolusheva, S., Boyer, L. \& Jelinek, R. A colorimetric assay for rapid screening of antimicrobial peptides. Nat. Biotechnol. 18, 225-227 (2000).

35. Briand, E., Zäch, M., Svedhem, S., Kasemo, B. \& Petronis, S. Combined QCM-D and EIS study of supported lipid bilayer formation and interaction with pore-forming peptides. Analyst 135, 343-350 (2010). 
36. Tamm, L. K. \& McConnell, H. M. Supported phospholipid bilayers. Biophys. J. 47, 105113 (1985).

37. Sackmann, E. Supported Membranes: Scientific and Practical Applications. Science (80-. ). 271, 43 LP-- 48 (1996).

38. Menger, F. M. \& Keiper, J. S. Chemistry and physics of giant vesicles as biomembrane models. Curr. Opin. Chem. Biol. 2, 726-732 (1998).

39. Walde, P., Cosentino, K., Engel, H. \& Stano, P. Giant Vesicles: Preparations and Applications. ChemBioChem 11, 848-865 (2010).

40. Akashi, K., Miyata, H., Itoh, H. \& Kinosita, K. Preparation of giant liposomes in physiological conditions and their characterization under an optical microscope. Biophys. J. 71, 3242-3250 (1996).

41. Engelhardt, H., Duwe, H.P. \& Sackmann, E. Bilayer bending elasticity measured by Fourier analysis of thermally excited surface undulations of flaccid vesicles. J. Phys. Lett. 46, 395400 (1985).

42. Bivas, I., Hanusse, P., Bothorel, P., Lalanne, J. \& Aguerre-Chariol, O. An application of the optical microscopy to the determination of the curvature elastic modulus of biological and model membranes. J. Phys. Fr. 48, 855-867 (1987).

43. Evans, E. \& Rawicz, W. Entropy-driven tension and bending elasticity in condensed-fluid membranes. Phys. Rev. Lett. 64, 2094-2097 (1990).

44. Pan, J., Mills, T. T., Tristram-Nagle, S. \& Nagle, J. F. Cholesterol perturbs lipid bilayers nonuniversally. Phys. Rev. Lett. 100, 1-4 (2008).

45. Méléard, P. et al. Mechanical properties of model membranes studied from shape transformations of giant vesicles. Biochimie 80, 401-413 (1998).

46. Dimova, R. et al. A practical guide to giant vesicles. Probing the membrane nanoregime via optical microscopy. J. Phys. Condens. Matter 18, (2006).

47. Fernandez-Puente, L., Bivas, I., Mitov, M. D. \& Méléard, P. Temperature and Chain Length Effects on Bending Elasticity of Phosphatidylcholine Bilayers. Europhys. Lett. 28, 181-186 (2007).

48. Dimova, R., Pouligny, B. \& Dietrich, C. Pretransitional Effects in Dimyristoylphosphatidylcholine Vesicle Membranes: Optical Dynamometry Study. Biophys. J. 79, 340-356 (2000).

49. Nagle, J. F. Introductory lecture: Basic quantities in model biomembranes. Faraday Discuss. 161, 11-29 (2012).

50. Calò, A. et al. Force measurements on natural membrane nanovesicles reveal a composition-independent, high Young's modulus. Nanoscale 6, 2275-2285 (2014).

51. Li, S., Eghiaian, F., Sieben, C., Herrmann, A. \& Schaap, I. A. T. Bending and puncturing the influenza lipid envelope. Biophys. J. 100, 637-645 (2011). 
52. Manneville, J. B., Bassereau, P., Lévy, D. \& Prost, J. Activity of transmembrane proteins induces magnification of shape fluctuations of lipid membranes. Phys. Rev. Lett. 82, 43564359 (1999).

53. Girard, P., Prost, J. \& Bassereau, P. Passive or active fluctuations in membranes containing proteins. Phys. Rev. Lett. 94, 88102-5 (2005).

54. Dimova, R. Recent developments in the field of bending rigidity measurements on membranes. Adv. Colloid Interface Sci. 208, 225-234 (2014).

55. Vorselen, D., Dommelen, S. M. Van, Sorkin, R., Schiller, J. \& Wijk, R. Van. The Fluid Membrane determines Mechanics of Red Blood Cell Extracellular Vesicles and is Softened in Hereditary Spherocytosis. bioRxiv 212456 (2017).

56. Sorkin, R. et al. Stiffness of natural extra-cellular vesicles is gov- erned by membrane protein content. Eur. Biophys. J. 46, S128 (2017).

57. Harroun, T. A., Heller, W. T., Weiss, T. M., Yang, L. \& Huang, H. W. Experimental Evidence for Hydrophobic Matching and Membrane- Mediated Interactions in Lipid Bilayers Containing Gramicidin. Biophys. J. 76, 937-945 (1999).

58. Whitesides, G. M. The origins and the future of microfluidics. Nature 442, 368-373 (2006).

59. Reyes, D. R., Iossifidis, D., Auroux, P. A. \& Manz, A. Micro total analysis systems. 1. Introduction, theory, and technology. Anal. Chem. 74, 2623-2636 (2002).

60. Toner, M. \& Irimia, D. Blood-on-a-Chip. Annu. Rev. Biomed. Eng. 7, 77-103 (2005).

61. Suresh, S. et al. Connections between single-cell biomechanics and human disease states: Gastrointestinal cancer and malaria. Acta Biomater. 23, S3-S15 (2015).

62. Vaziri, A. \& Gopinath, A. Cell and biomolecular mechanics in silico. Nat. Mater. 7, 15-23 (2008).

63. Cranston, H. et al. Plasmodium falciparum maturation abolishes physiologic red cell deformability. Science (80-. ). 223, 400-403 (1998).

64. Bhagat, A. A. S. et al. Microfluidics for cell separation. Med. Biol. Eng. Comput. 48, 9991014 (2010).

65. Gossett, D. R. et al. Label-free cell separation and sorting in microfluidic systems. Anal. Bioanal. Chem. 397, 3249-3267 (2010).

66. Lenshof, A. \& Laurell, T. Continuous separation of cells and particles in microfluidic systems. Chem. Soc. Rev. 39, 1203-1217 (2010).

67. Pamme, N. Continuous flow separations in microfluidic devices. Lab Chip 7, 1644-1659 (2007).

68. Oakey, J., Allely, J. \& Marr, D. W. M. Laminar-flow-based separations at the microscale. Biotechnol. Prog. 18, 1439-1442 (2002).

69. Yamada, M., Nakashima, M. \& Seki, M. Pinched flow fractionation: Continuous size separation of particles utilizing a laminar flow profile in a pinched microchannel. Anal. Chem. 76, 5465-5471 (2004). 
70. Park, J. S. \& Jung, H. Il. Multiorifice flow fractionation: Continuous size-based separation of microspheres using a series of contraction/expansion microchannels. Anal. Chem. 81, 8280-8288 (2009).

71. Asmolov, E. S. The inertial lift on a spherical particle in plane Poiseuille flow at large Reynolds number. J. Fluid Mech. 381, 63-87 (1999).

72. Zeng, L., Balachandar, S. \& Fischer, P. Wall-induced forces on a rigid sphere at finite Reynolds number. J. Fluid Mech. 536, 1-25 (2005).

73. Segré, G. \& Silberberg, A. Radial particle displacements in poiseuille flow of suspensions. Nature 189, 209-210 (1961).

74. Lee, M. G., Choi, S. \& Park, J. K. Inertial separation in a contraction-expansion array microchannel. J. Chromatogr. A 1218, 4138-4143 (2011).

75. Hsua, C.-H., Carloa, D. Di, Chena, C., Irimiaa, D. \& Toner, M. Microvortex for focusing, guiding and sorting of particles. Lab Chip 8, 2128-2134 (2008).

76. Huang, L. R., Cox, E. C., Austin, R. H. \& Sturm, J. C. Continous particle separation through deterministic lateral displacement. Science (80-. ). 304, 987-991 (2004).

77. Long, B. R. et al. Multidirectional sorting modes in deterministic lateral displacement devices. Phys. Rev. E - Stat. Nonlinear, Soft Matter Phys. 78, (2008).

78. Beech, J. P. Mocrofluidics Separation and Analysis of Biological Particles. (2011).

79. Balvin, M., Sohn, E., Iracki, T., Drazer, G. \& Frechette, J. Directional locking and the role of irreversible interactions in deterministic hydrodynamics separations in microfluidic Devices. Phys. Rev. Lett. 103, 2-5 (2009).

80. Inglis, D. W., Davis, J. A., Austin, R. H. \& Sturm, J. C. Critical particle size for fractionation by deterministic lateral displacement. Lab Chip 6, 655-658 (2006).

81. Loutherback, K. et al. Deterministic separation of cancer cells from blood at $10 \mathrm{~mL} / \mathrm{min}$ Deterministic separation of cancer cells from blood at $10 \mathrm{~mL} / \mathrm{min}$. AIP Adv. 42107, 1-8 (2012).

82. Holm, S. H., Beech, J. P., Barrett, M. P. \& Tegenfeldt, J. O. Separation of parasites from human blood using deterministic lateral displacement. Lab Chip 11, 1326-1332 (2011).

83. Joensson, H. N., Uhlén, M. \& Svahn, H. A. Deterministic Lateral Displacement Device for Droplet Separation By Size - Towards Rapid Clonal Selection Based on Droplet Shrinking. 14th Int. Conf. Miniaturized Syst. Chem. Life Sci. 3 - 7 Oct. 2010, Groningen, Netherlands 1355-1357 (2010).

84. Yang, S., Ündar, A. \& Zahn, J. D. A microfluidic device for continuous, real time blood plasma separation. Lab Chip 6, 871-880 (2006).

85. Redkar, S. G. \& Davis, R. H. Cross-flow microfiltration with high-frequency reverse filtration. AIChE J. 41, 501-508 (1995).

86. Aran, K. et al. Microfiltration platform for continuous blood plasma protein extraction from whole blood during cardiac surgery. Lab Chip 11, 2858-2868 (2011). 
87. Lo, M. \& Zahn, J. D. Development of a Multi-Compartment Microfiltration Device for Particle Fractionation. 16th Int. Conf. Miniaturized Syst. Chem. Life Sci. Oct. 28 - Novemb. 1, 2012, Okinawa, Japan 527-529 (2012).

88. Murthy, S. K., Sethu, P., Vunjak-Novakovic, G., Toner, M. \& Radisic, M. Size-based microfluidic enrichment of neonatal rat cardiac cell populations. Biomed. Microdevices 8, 231-237 (2006).

89. VanDelinder, V. \& Groisman, A. Perfusion in microfluidic cross-flow: Separation of white blood cells from whole blood and exchange of medium in a continuous flow. Anal. Chem. 79, 2023-2030 (2007).

90. Crowley, T. A. \& Pizziconi, V. Isolation of plasma from whole blood using planar microfilters for lab-on-a-chip applications. Lab Chip 5, 922-929 (2005).

91. Ripperger, S. \& Altmann, J. Crossflow microfiltration - State of the art. Sep. Purif. Technol. 26, 19-31 (2002).

92. Yamada, M. \& Seki, M. Hydrodynamic filtration for on-chip particle concentration and classification utilizing microfluidics. Lab Chip 5, 1233-1239 (2005).

93. Yamada, M. \& Seki, M. Microfluidic particle sorter employing flow splitting and recombining. Anal. Chem. 78, 1357-1362 (2006).

94. Bhardwaj, P., Bagdi, P. \& Sen, A. K. Lab on a Chip Microfluidic device based on a microhydrocyclone for particle - liquid separation. 4012-4021 (2011). doi:10.1039/c1lc20606k

95. Gascoyne, P. R. C. \& Vykoukal, J. Particle separation by dielectrophoresis. Electrophoresis 23, 1973-1983 (2002).

96. Holmes, D. \& Morgan, H. Cell positioning and sorting using dielectrophoresis. Eur. Cells Mater. 4, 120-122 (2002).

97. $\mathrm{Hu}, \mathrm{X}$. et al. Marker-specific sorting of rare cells using dielectrophoresis. Proc. Natl. Acad. Sci. 102, 15757-15761 (2005).

98. Kersaudy-Kerhoas, M., Dhariwal, R., Desmulliez, M. P. Y. \& Jouvet, L. Hydrodynamic blood plasma separation in microfluidic channels. Microfluid. Nanofluidics 8, 105-114 (2010).

99. Miltenyi, S., Müller, W., Weichel, W. \& Radbruch, A. High gradient magnetic cell separation with MACS. Cytometry 11, 231-8 (1990).

100. Adams, J. D., Kim, U. \& Soh, H. T. Multitarget magnetic activated cell sorter. Proc. Natl. Acad. Sci. 105, 18165-18170 (2008).

101. Gooneratne, C. P. \& Kosel, J. A micro-pillar array to trap magnetic beads in microfluidic systems. Proc. Int. Conf. Sens. Technol. ICST 97-101 (2012). doi:10.1109/ICSensT.2012.6461798

102. Gijs, M. A. M. Magnetic bead handling on-chip: New opportunities for analytical applications. Microfluid. Nanofluidics 1, 22-40 (2004). 
103. Pamme, N., Eijkel, J. C. T. \& Manz, A. On-chip free-flow magnetophoresis: Separation and detection of mixtures of magnetic particles in continuous flow. J. Magn. Magn. Mater. 307, 237-244 (2006).

104. McGloin, D. Optical tweezers: 20 Years on. Philos. Trans. R. Soc. A Math. Phys. Eng. Sci. 364, 3521-3537 (2006).

105. Ashkin, A., Dziedzic, J. M., Bjorkholm, J. E. \& Chu, S. Observation of a single-beam gradient force optical trap for dielectric particles. Opt. Lett. 11, 288 (1986).

106. Ashkin, A. Optical Trapping and Manipulation of Neutral Particles Using Lasers. Opt. Photonics News 10, 41 (1997).

107. Lee, M. P. \& Padgett, M. J. Optical tweezers: A light touch. J. Microsc. 248, 219-222 (2012).

108. Reicherter, M., Haist, T., Wagemann, E. U. \& Tiziani, H. J. Optical particle trapping with computer-generated holograms written on a liquid-crystal display. Opt. Lett. 24, 608 (1999).

109. MacDonald, M. P., Spalding, G. C. \& Dholakia, K. Microfluidic sorting in an optical lattice. Nature 426, 421-424 (2003).

110. Liesfeld, B., Nambiar, R. \& Meiners, J. C. Particle transport in asymmetric scanning-line optical tweezers. Phys. Rev. E - Stat. Nonlinear, Soft Matter Phys. 68, 519071-519076 (2003).

111. Ma, B. et al. Optical sorting of particles by dual-channel line optical tweezers. J. Opt. (United Kingdom) 14, (2012).

112. Zheng, M. J., Qu, Y. L., Zhang, Y. Z. \& Dong, Z. L. Optically induced dielectrophoresis based automatic assembly of micro/nano-devices. Integr. Ferroelectr. 145, $24-31$ (2013).

113. Kaneta, T., Ishidzu, Y., Mishima, N. \& Imasaka, T. Theory of Optical Chromatography. Anal. Chem. 69, 2701-2710 (1997).

114. Monjushiro, H., Hirai, A. \& Watarai, H. Size dependence of laser-photophoretic efficiency of polystyrene microparticles in water. Langmuir 16, 8539-8542 (2000).

115. Xin, H., Lei, H., Zhang, Y., Li, X. \& Li, B. Photothermal trapping of dielectric particles by optical fiber-ring. Opt. Express 19, 2711 (2011).

116. Soong, C. Y., Li, W. K., Liu, C. H. \& Tzeng, P. Y. Theoretical analysis for photophoresis of a microscale hydrophobic particle in liquids. Opt. Express 18, 2168-2182 (2010).

117. Leibacher, I., Reichert, P. \& Dual, J. Microfluidic droplet handling by bulk acoustic wave (BAW) acoustophoresis. Lab Chip 15, 2896-2905 (2015).

118. Wu, Z., Liu, A. Q. \& Hjort, K. Microfluidic continuous particle/cell separation via electroosmotic-flow- tuned hydrodynamic spreading. J. Micromechanics Microengineering 17, 1992-1999 (2007).

119. Lee, K., Kim, S. S. \& Sung, H. J. ADJUSTABLE PARTICLE SEPARATION IN PINCHED. (2010).

120. Beech, J. P. \& Tegenfeldt, J. O. Tipping the balance with dielectro- phoretic forces - an electric determi- nistic lateral displacement device. Lab Chip 95-97 (2008). 
121. Groschl, M. Ultrasonic Separation of Suspended Particles - Part I : Fundamentals. Acustica 84, 432-447 (1998).

122. Lilliehorn, T. et al. Trapping of microparticles in the near field of an ultrasonic transducer. Ultrasonics 43, 293-303 (2005).

123. Bazou, D., Kuznetsova, L. A. \& Coakley, W. T. Physical enviroment of 2-D animal cell aggregates formed in a short pathlength ultrasound standing wave trap. Ultrasound Med. Biol. 31, 423-430 (2005).

124. Encyclopedia of Microfluidics and Nanofluidics. (Springer US, 2008). doi:10.1007/978-0387-48998-8

125. Bruus, H. Acoustofluidics 7: The acoustic radiation force on small particles. Lab Chip 12, 1014-1021 (2012).

126. Johnson, D. A. \& Feke, D. L. Methodology for fractionating suspended particles using ultrasonic standing wave and divided flow fields. Sep. Technol. 5, 251-258 (1995).

127. Yasuda, K., Umemura, S. \& Takeda, K. Concentration and Fractionation of Small Particles in Liquid by Ultrasound. Jpn. J. Appl. Phys. 34, 2715 (1995).

128. Petersson, F., Åberg, L., Swärd-Nilsson, A. M. \& Laurell, T. Free flow acoustophoresis: Microfluidic-based mode of particle and cell separation. Anal. Chem. 79, 5117-5123 (2007).

129. Dolatmoradi, A. Thermally-Assisted Acoustofluidic Separation for Bioanalytical Applications. (Florida International University, 2017).

130. Bruus, H. Acoustofluidics 2: Perturbation theory and ultrasound resonance modes. Lab Chip 12, 20-28 (2012).

131. Doblhoff-Dier, O. et al. A Novel Ultrasonic Resonance Field Device for the Retention of Animal Cells. Biotechnol. Prog. 10, 428-432 (1994).

132. Hawkes, J. J. \& Coakley, W. T. Force field particle filter, combining ultrasound standing waves and laminar flow. Sensors Actuators B Chem. 75, 213-222 (2001).

133. Petersson, F., Nilsson, A., Jönsson, H. \& Laurell, T. Carrier Medium Exchange through Ultrasonic Particle Switching in Microfluidic Channels. Anal. Chem. 77, 1216-1221 (2005).

134. Kuznetsova, L. A. \& Coakley, W. T. Applications of ultrasound streaming and radiation force in biosensors. Biosens. Bioelectron. 22, 1567-1577 (2007).

135. Martin, S. P. et al. Spore and micro-particle capture on an immunosensor surface in an ultrasound standing wave system. Biosens. Bioelectron. 21, 758-767 (2005).

136. Laurell, T., Petersson, F. \& Nilsson, A. Chip integrated strategies for acoustic separation and manipulation of cells and particles. Chem. Soc. Rev. 36, 492-506 (2007).

137. Burguillos, M. a. et al. Microchannel Acoustophoresis does not Impact Survival or Function of Microglia, Leukocytes or Tumor Cells. PLoS One 8, 1-11 (2013).

138. Thévoz, P., Adams, J. D., Shea, H., Bruus, H. \& Soh, H. T. Acoustophoretic synchronization of mammalian cells in microchannels. Anal. Chem. 82, 3094-3098 (2010). 
139. Yang, A. H. J. \& Soh, H. T. Acoustophoretic Sorting of Viable Mammalian Cells in a Microfluidic Device. Anal. Chem. 84, 10756-10762 (2012).

140. Grenvall, C., Magnusson, C., Lilja, H. \& Laurell, T. Concurrent Isolation of Lymphocytes and Granulocytes Using Prefocused Free Flow Acoustophoresis. Anal. Chem. 5596-5604 (2015). doi:10.1021/acs.analchem.5b00370

141. Ward, M., Turner, P., DeJohn, M. \& Kaduchak, G. Fundamentals of acoustic cytometry. Curr. Protoc. Cytom. 1-12 (2009). doi:10.1002/0471142956.cy0122s49

142. Hawkes, J. J., Barber, R. W., Emerson, D. R. \& Coakley, W. T. Continuous cell washing and mixing driven by an ultrasound standing wave within a microfluidic channel. Lab Chip 4, 446-452 (2004).

143. Tenje, M. et al. Acoustophoretic removal of proteins from blood components. Biomed. Microdevices 17, 95 (2015).

144. Antfolk, M., Antfolk, C., Lilja, H., Laurell, T. \& Augustsson, P. A single inlet two-stage acoustophoresis chip enabling tumor cell enrichment from white blood cells. Lab Chip 15, 2102-2109 (2015).

145. Weinberg, R. A. The biology of Cancer. (Garland Science, Taylor \& Francis Group, LLC, 2014).

146. Li, P. et al. Acoustic separation of circulating tumor cells. Proc. Natl. Acad. Sci. U. S. A. 112, 4970-4975 (2015).

147. Ding, X. et al. Cell separation using tilted-angle standing surface acoustic waves. Proc. Natl. Acad. Sci. 111, 12992-12997 (2014).

148. Shields, C. W., Johnson, L. M., Gao, L. \& López, G. P. Elastomeric Negative Acoustic Contrast Particles for Capture, Acoustophoretic Transport, and Con fi nement of Cells in Micro fl uidic Systems. Langmuir 30, 3923-3927 (2014).

149. Lenshof, A., Evander, M., Laurell, T. \& Nilsson, J. Acoustofluidics 5: Building microfluidic acoustic resonators. Lab Chip 12, 684-695 (2012).

150. Gao, K.-J. et al. Giant vesicle formation through self-assembly of chitooligosaccharidebased graft copolymers. Chem. Commun. 1449-1451 (2008). doi:10.1039/B718117E

151. Batzri, S. \& Korn, E. D. Single bilayer liposomes prepared without sonication. Biochim. Biophys. Acta 298, 1015-1019 (1973).

152. Pons, M., Foradada, M. \& Estelrich, J. Liposomes obtained by the ethanol injection method. Int. J. Pharm. 95, 51-56 (1993).

153. Purrucker, O., Hillebrandt, H., Adlkofer, K. \& Tanaka, M. Deposition of highly resistive lipid bilayer on silicon-silicon dioxide electrode and incorporation of gramicidin studied by ac impedance spectroscopy. Electrochim. Acta 47, 791-798 (2001).

154. Gritsch, S., Nollert, P., Jähnig, F. \& Sackmann, E. Impedance spectroscopy of porin and gramicidin pores reconstituted into supported lipid bilayers on indium-tin-oxide electrodes. Langmuir 14, 3118-3125 (1998). 
155. Whitehouse, C., Gidalevitz, D., Cahuzac, M., Koeppe, R. E. \& Nelson, A. Interaction of gramicidin derivatives with phospholipid monolayers. Langmuir 20, 9291-9298 (2004).

156. Bourinbaiar, A. S. \& Coleman, C. F. The effect of gramicidin, a topical contraceptive and antimicrobial agent with anti-HIV activity, against herpes simplex viruses type 1 and 2 in vitro. Arch. Virol. 142, 2225-2235 (1997).

157. Burkhart, B. M. et al. Gramicidin D Conformation, Dynamics and Membrane. Biopolym. (Peptide Sci. 51, 129-144 (1999).

158. Okoro, L. N. Effect of Gramicidin D on the Compressibility and Volume Fluctuations of DPPC - Peptide Bilayers: A Densitometry and Sound Velocimetry Study. Int. J. Chem. 3, 166-175 (2011).

159. Marsh, D. Orientation and peptide-lipid interactions of alamethicin incorporated in phospholipid membranes: Polarized infrared and spin-label EPR spectroscopy. Biochemistry 48, 729-737 (2009).

160. Huang, H. W. \& Wu, Y. Lipid-alamethicin interactions influence alamethicin orientation. Biophys. J. 60, 1079-1087 (1991).

161. Greenspan, P., Mayer, E. P. \& Fowler, S. D. Nile red: a selective fluorescent stain for intracellular lipid droplets. J. Cell Biol. 100, 965 LP-- 973 (1985).

162. Niel, G. van, D'Angelo, G. \& Raposo, G. Shedding light on the cell biology of extracellular vesicles. Nat. Rev. Mol. Cell Biol. 19, 213-228 (2018).

163. Keller, S. et al. Systemic presence and tumor-growth promoting effect of ovarian carcinoma released exosomes. Cancer Lett. 278, 73-81 (2009).

164. Robbins, P. D. \& Morelli, A. E. Regulation of Immune Responses by Extracellular Vesicules. Nat. Immunol. 14, 195-208 (2014).

165. Adachi, J., Kumar, C., Zhang, Y., Olsen, J. V \& Mann, M. The human urinary proteome contains more than 1500 proteins, including a large proportion of membrane proteins. Genome Biol. 7, R80 (2006).

166. Mitchell, P. J. et al. Can urinary exosomes act as treatment response markers in prostate cancer? J. Transl. Med. 7, (2009).

167. Nilsson, J. et al. Prostate cancer-derived urine exosomes: a novel approach to biomarkers for prostate cancer. Br. J. Cancer 100, 1603-1607 (2009).

168. Pisitkun, T., Shen, R. F. \& Knepper, M. A. Identification and proteomic profiling of exosomes in human urine. Proc Natl Acad Sci USA 101, 13368-13373 (2004).

169. Simpson, R. J., Jensen, S. S. \& Lim, J. W. E. Proteomic profiling of exosomes: Current perspectives. Proteomics 8, 4083-4099 (2008).

170. Zhou, H. et al. Collection, storage, preservation, and normalization of human urinary exosomes for biomarker discovery. Kidney Int. 69, 1471-1476 (2006).

171. Karachaliou, N., Mayo-de-las-casas, C., Molina-vila, M. A. \& Rosell, R. Real-time liquid biopsies become a reality in cancer treatment. Ann. Transl. Med. 3, 36 (2015). 
172. Torrano, V. et al. Vesicle-MaNiA: Extracellular vesicles in liquid biopsy and cancer. Curr. Opin. Pharmacol. 29, 47-53 (2016).

173. Cesi, G., Walbrecq, G., Margue, C. \& Kreis, S. Transferring intercellular signals and traits between cancer cells: extracellular vesicles as 'homing pigeons'. Cell Commun. Signal. 14, (2016).

174. Guo, S. C., Tao, S. C. \& Dawn, H. Microfluidics-based on-a-chip systems for isolating and analysing extracellular vesicles. J. Extracell. Vesicles 7, (2018).

175. Contreras-Naranjo, J. C., Wu, H. J. \& Ugaz, V. M. Microfluidics for exosome isolation and analysis: Enabling liquid biopsy for personalized medicine. Lab Chip 17, 3558-3577 (2017).

176. Zaborowski, M. P., Balaj, L., Breakefield, X. O. \& Lai, C. P. Extracellular Vesicles: Composition, Biological Relevance, and Methods of Study. Bioscience 65, 783-797 (2015).

177. Li, P., Kaslan, M., Lee, S. H., Yao, J. \& Gao, Z. Progress in exosome isolation techniques. Theranostics 7, 789-804 (2017).

178. Llorente, A. et al. Molecular lipidomics of exosomes released by PC-3 prostate cancer cells. Biochim. Biophys. Acta - Mol. Cell Biol. Lipids 1831, 1302-1309 (2013).

179. Lydic, T. A. et al. Rapid and comprehensive 'shotgun' lipidome profiling of colorectal cancer cell derived exosomes. Methods 87, 83-95 (2015).

180. Park, J. et al. Acoustothermal tweezer for droplet sorting in a disposable microfluidic chip. Lab Chip 17, 1031-1040 (2017).

181. $\mathrm{Wu}, \mathrm{M}$. et al. Isolation of exosomes from whole blood by integrating acoustics and microfluidics. Proc. Natl. Acad. Sci. 114, 10584-10589 (2017).

182. Rezeli, M. et al. Comparative Proteomic Analysis of Extracellular Vesicles Isolated by Acoustic Trapping or Differential Centrifugation. Anal. Chem. 88, 8577-8586 (2016).

183. $\mathrm{Ku}$, A. et al. Acoustic Enrichment of Extracellular Vesicles from Biological Fluids. Anal. Chem. 90, 8011-8019 (2018).

184. Bryl-Górecka, P. et al. Effect of exercise on the plasma vesicular proteome: A methodological study comparing acoustic trapping and centrifugation. Lab Chip 18, 31013111 (2018).

185. Dolatmoradi, A. \& El-Zahab, B. Thermally-assisted ultrasonic separation of giant vesicles. Lab Chip 16, 3449-3453 (2016).

186. Dolatmoradi, A., Mirtaheri, E. \& El-Zahab, B. Thermo-Acoustofluidic Separation of Vesicles Based on Cholesterol Content. Lab Chip 17, 1332-1339 (2017).

187. Mirtaheri, E., Dolatmoradi, A. \& El-Zahab, B. Thermally-assisted acoustofluidic separation based on membrane protein content. Anal. Chem. (2019). doi:10.1021/acs.analchem.9b03485

188. Andersen, O. S., Koeppe, R. E. \& Roux, B. Gramicidin channels. IEEE Trans. Nanobioscience 4, 10-19 (2005). 
189. Townsley, L. E., Tucker, W. A., Sham, S. \& Hinton, J. F. Structures of gramicidins A, B, and C incorporated into sodium dodecyl sulfate micelles. Biochemistry 40, 11676-11686 (2001).

190. Wallace, B. A. Recent Advances in the High Resolution Structures of Bacterial Channels: Gramicidin A. J. Struct. Biol. 121, 123-41 (1998).

191. Kelkar, D. A. \& Chattopadhyay, A. The gramicidin ion channel: A model membrane protein. Biochim. Biophys. Acta - Biomembr. 1768, 2011-2025 (2007).

192. Woolf, T. B. \& Roux, B. Molecular Dynamics Simulation of the Gramicidin Channel in a Phospholipid Bilayer. PNAS 91, 11631-11635 (1994).

193. Lewis, R. N. A. H. et al. Fourier transform infrared spectroscopic studies of the interaction of the antimicrobial peptide gramicidin $\mathrm{S}$ with lipid micelles and with lipid monolayer and bilayer membranes. Biochemistry 38, 15193-15203 (1999).

194. Zein, M. \& Winter, R. Effect of temperature, pressure and lipid acyl chain length on the structure and phase behavior of phospholipid-gramicidin bilayers. Phys Chem Chem Phys 2, 4545-4551 (2000).

195. Ge, M. \& Freed, J. H. Electron-spin resonance study of aggregation of gramicidin in dipalmitoylphosphatidylcholine bilayers and hydrophobic mismatch. Biophys J 76, 264280 (1999).

196. Vitkova, V., Méléard, P., Pott, T. \& Bivas, I. Alamethicin influence on the membrane bending elasticity. Eur. Biophys. J. 35, 281-286 (2006).

197. Basu, I., Chattopadhyay, A. \& Mukhopadhyay, C. Ion channel stability of Gramicidin A in lipid bilayers: Effect of hydrophobic mismatch. Biochim. Biophys. Acta - Biomembr. 1838, 328-338 (2014).

198. Pan, J., Tieleman, D. P., Nagle, J. F., Kucerka, N. \& Tristram-Nagle, S. Alamethicin in Lipid Bilayers: Combined use of X-ray Scattering and MD Simulations. 1788, 1387-97 (2009).

199. Pabst, G., Danner, S., Podgornik, R. \& Katsaras, J. Entropy-driven softening of fluid lipid bilayers by alamethicin. Langmuir 23, 11705-11711 (2007).

200. Cornell, B. A., Weir, L. E. \& Separovic, F. The effect of gramicidin A on phospholipid bilayers. Eur. Biophys. J. 16, 113-119 (1988).

201. Harroun, T. A., Heller, W. T., Weiss, T. M., Yang, L. \& Huang, H. W. Theoretical analysis of hydrophobic matching and membrane-mediated interactions in lipid bilayers containing gramicidin. Biophys. J. 76, 3176-3185 (1999).

202. Guo, Y., Pogodin, S. \& Baulin, V. A. General model of phospholipid bilayers in fluid phase within the single chain mean field theory. J. Chem. Phys. 140, (2014).

203. Diociaiuti, M. et al. Aggregation of gramicidin A in phospholipid Langmuir-Blodgett monolayers. Biophys. J. 82, 3198-3206 (2002).

204. Costa-Filho, A. J., Crepeau, R. H., Borbat, P. P., Ge, M. \& Freed, J. H. Lipid-gramicidin interactions: Dynamic structure of the boundary lipid by 2D-ELDOR. Biophys. J. 84, 33643378 (2003). 
205. Hanke, W. et al. Melittin and a chemically modified trichotoxin form alamethicin-type multi-state pores. BBA - Biomembr. 727, 108-114 (1983).

206. Dawson, C. R., Drake, A. F., Helliwell, J. \& Hider, R. C. the interaction of Bee Melittin With Lipid Bilayer. Biochim. Biophys. Acta 0, 75-86 (1978).

207. Sharma, V. K. et al. Antimicrobial Peptide Impacts the Lateral Diffusion and Bending Rigidity of Phospholipid Membrane. Biophys. J. 110, 246a (2016).

208. Théry, C., Zitvogel, L. \& Amigorena, S. Exosomes: composition, biogenesis and function. Nat. Rev. Immunol. 2, 569-579 (2002).

209. Wiklund, M. Acoustofluidics 12: Biocompatibility and cell viability in microfluidic acoustic resonators. Lab Chip 12, 2018-2028 (2012).

210. Melchior, D. L. \& Steim, J. M. Thermotropic transitions in biomembranes. Annu. Rev. Biophys. Bioeng. 5, 205-238 (1976).

211. Brasitus, T. A., Schachter, D. \& Mamouneas, T. G. Functional Interactions of Lipids and Proteins in Rat Intestinal Microvillus Membranes. Biochemistry 18, 4136-4144 (1979).

212. Barnkob, R., Augustsson, P., Laurell, T. \& Bruus, H. Acoustic radiation- and streaminginduced microparticle velocities determined by microparticle image velocimetry in an ultrasound symmetry plane. Phys. Rev. E - Stat. Nonlinear, Soft Matter Phys. 86, 1-11 (2012).

213. Wiklund, M., Green, R. \& Ohlin, M. Acoustofluidics 14: Applications of acoustic streaming in microfluidic devices. Lab Chip 12, 2438-2451 (2012).

214. Sehgal, P. \& Kirby, B. J. Separation of 300 and $100 \mathrm{~nm}$ Particles in Fabry-Perot Acoustofluidic Resonators. Anal. Chem. 89, 12192-12200 (2017).

215. Tacn, A. Melittin and Cancer. J. Apitherapy 1, 51 (2016).

216. Son, D. J. et al. Therapeutic application of anti-arthritis, pain-releasing, and anti-cancer effects of bee venom and its constituent compounds. Pharmacol. Ther. 115, 246-270 (2007).

217. Gajski, G. \& Garaj-Vrhovac, V. Melittin: A lytic peptide with anticancer properties. Environ. Toxicol. Pharmacol. 36, 697-705 (2013).

218. Pan, H., Soman, N. R., Schlesinger, P. H., Lanza, G. M. \& Wickline, S. A. Cytolytic peptide nanoparticles ('NanoBees') for cancer therapy. Wiley Interdiscip. Rev. Nanomedicine Nanobiotechnology 3, 318-327 (2011).

219. Hur, J., Kim, K., Lee, S., Park, H. \& Park, Y. Melittin-induced alterations in morphology and deformability of human red blood cells using quantitative phase imaging techniques. Sci. Rep. 7, 1-10 (2017).

220. Adachi, J., Kumar, C., Zhang, Y., Olsen, J. V \& Mann, M. The human urinary proteome contains more than 1500 proteins, including a large proportion of membrane proteins. Genome Biol. 7, R80 (2006). 
221. Attard, G. et al. Characterization of ERG, AR and PTEN gene status in circulating tumor cells from patients with castration-resistant prostate cancer. Cancer Res 69, 2912-2918 (2009).

222. Smalheiser, N. R. Exosomal transfer of proteins and RNAs at synapses in the nervous system. Biol Direct 2, (2007).

223. Jansen, F. H. et al. Exosomal secretion of cytoplasmic prostate cancer xenograft-derived proteins. Mol Cell Proteomics 8, 1192-1205 (2009).

224. Keller, S. et al. Systemic presence and tumor-growth promoting effect of ovarian carcinoma released exosomes. Cancer Lett. 278, 73-81 (2009).

225. Kim, J. W. et al. Fas Ligand - Positive Membranous Vesicles Isolated from Sera of Patients with Oral Cancer Induce Apoptosis of Activated T Lymphocytes Fas Ligand - Positive Membranous Vesicles Isolated from Sera of Patients with Oral Cancer Induce Apoptosis of Activated T. Clin. Cancer Res. 11, 1010-1020 (2005).

226. Logozzi, M. et al. High levels of exosomes expressing CD63 and caveolin-1 in plasma of melanoma patients. PLoS One 4, (2009).

227. Feng, Q. et al. A class of extracellular vesicles from breast cancer cells activates VEGF receptors and tumour angiogenesis. Nat. Commun. 8, 14450 (2017).

228. Maas, S. L. N. et al. Possibilities and limitations of current technologies for quantification of biological extracellular vesicles and synthetic mimics. J. Control. Release 200, 87-96 (2015).

229. Chen, Y. \& Hughes-Fulford, M. Human prostate cancer cells lack feedback regulation of low-density lipoprotein receptor and its regulator, SREBP2. Int. J. Cancer 91, 41-45 (2001).

230. Li, Y. C., Park, M. J., Ye, S.-K., Kim, C.-W. \& Kim, Y.-N. Elevated Levels of CholesterolRich Lipid Rafts in Cancer Cells Are Correlated with Apoptosis Sensitivity Induced by Cholesterol-Depleting Agents. Am. J. Pathol. 168, 1107-1118 (2018). 
VITA

\section{ELNAZ MIRTAHERI}

1986

Born, Qazvin, Iran

2008

B.S., Materials Engineering

Imam Khomeini International University

Qazvin, Iran

2011

M.S., Materials Engineering

University of Tehran

Tehran, Iran

2011-2014

Senior/Staff Materials Engineer

Turbine Machine Middle East Corporation

Tehran, Iran

2015-2018

Graduate Teaching Assistant

Florida International University,

Miami, Florida

2018

NIH RISE Biomedical Research Initiative Award

Florida International University,

Miami, Florida

2016-2019

Doctoral Candidate in Materials Science and Engineering Florida International University, Miami, Florida

2018-2019

Doctoral Candidate and Dissertation Year Fellow

Florida International University,

Miami, Florida

2017

Provost Award for Best Poster in Engineering Category

Florida International University

Miami, Florida

2018

SPIE Student Travel Grant

Photonics West

San Francisco, California 


\section{PUBLICATIONS AND PRESENTATIONS}

N. Munroe, E. Mirtaheri, Polymer coated biodegradable stent material and methods of use, United States Patents, Grant (US 10046094B1), Aug. 2018.

E. Mirtaheri, A. Dolatmoradi, B. El-Zahab, Acoustofluidic separation based on membrane protein content, Analytical Chemistry, Accepted (2019)

A. Dolatmoradi, E. Mirtaheri, B. El-Zahab, Thermo-acoustofluidic separation of vesicles based on cholesterol content, Lab on a Chip, 2017, 17, 1332.

E. Mirtaheri, A. Dolatmoradi, K. Pimentel, S. Bhansali, B. El-Zahab, Thermally-assisted acoustofluidic separation of extracellular vesicles from cells, International Society for Optics and Photonics, Microfluidics, BioMEMS, and Medical Microsystems XVI, 2018, 10491, 1049131-6.

N. Munroe, V. Musaramthota, E. Mirtaheri, Surface modification and biological response of novel biomaterials for medical devices, SurFACTS in Biomaterials, 06/2015; 20(2).

E. Mirtaheri, N. Munroe, Improving biocompatibility of a magnesium stent alloy using a polymer coating, Poster presented at the 2016 Biointerface workshop and symposium, Oct. 2016, Minneapolis, Minnesota.

N. Munroe, E. Mirtaheri, V. Musaramthota, Tailoring the corrosion and biological response of novel biomaterials, Paper presented at the 1st International Workshop on NanoBio Surfaces and Interfaces in Healthcare and Science, Sep. 2016, Enschede, Netherlands.

E. Mirtaheri, N. Munroe, Improving the corrosion resistance of an $\mathrm{Mg}$ alloy by polymer coating, Poster presented at the Florida Statewide Graduate Research Symposium, Apr. 2016, Gainesville, Florida.

E. Mirtaheri, S. Amruthaluri, V. Musaramthota, N. Munroe, Effect of anodization on platelet adhesion of polymer coated $\mathrm{Mg}-1 \mathrm{Zn}-1 \mathrm{Ca}$ Alloy, Paper presented at the 8th Symposium on Biodegradable Metals, May 2016, Montréal, Canada. 